\title{
Bio-Based Polymer Electrolytes for Electrochemical Devices: Insight into the Ionic Conductivity Performance
}

\author{
Marwah Rayung ${ }^{1}$, Min Min Aung $1,2, * \mathbb{0}$, Shah Christirani Azhar ${ }^{2}$, Luqman Chuah Abdullah ${ }^{3}(\mathbb{D}$, \\ Mohd Sukor Su'ait ${ }^{4}$, Azizan Ahmad ${ }^{4,5}$ and Siti Nurul Ain Md Jamil ${ }^{2} \mathbb{C}$ \\ 1 Institute of Tropical Forestry and Forest Products, Universiti Putra Malaysia, Serdang 43400, Malaysia; \\ marwahrayung@yahoo.com \\ 2 Unit Chemistry, Center of Foundation Studies and Agricultural Science, Universiti Putra Malaysia, \\ Serdang 43400, Malaysia; christirani@upm.edu.my (S.C.A.); ctnurulain@upm.edu.my (S.N.A.M.J.) \\ 3 Department of Chemical and Environmental Engineering, Faculty of Engineering, Universiti Putra Malaysia, \\ Serdang 43400, Malaysia; chuah@upm.edu.my \\ 4 Solar Energy Research Institute (SERI), Universiti Kebangsaan Malaysia, Bangi 43600, Malaysia; \\ mohdsukor@ukm.edu.my (M.S.S.); azizan@ukm.edu.my (A.A.) \\ 5 School of Chemical Sciences and Food Technology, Universiti Kebangsaan Malaysia, Bangi 43600, Malaysia \\ * Correspondence: minmin_aung@upm.edu.my
}

Received: 10 October 2019; Accepted: 4 December 2019; Published: 12 February 2020

check for updates

\begin{abstract}
With the continuing efforts to explore alternatives to petrochemical-based polymers and the escalating demand to minimize environmental impact, bio-based polymers have gained a massive amount of attention over the last few decades. The potential uses of these bio-based polymers are varied, from household goods to high end and advanced applications. To some extent, they can solve the depletion and sustainability issues of conventional polymers. As such, this article reviews the trends and developments of bio-based polymers for the preparation of polymer electrolytes that are intended for use in electrochemical device applications. A range of bio-based polymers are presented by focusing on the source, the general method of preparation, and the properties of the polymer electrolyte system, specifically with reference to the ionic conductivity. Some major applications of bio-based polymer electrolytes are discussed. This review examines the past studies and future prospects of these materials in the polymer electrolyte field.
\end{abstract}

Keywords: bio-based polymer; polymer electrolyte; ionic conductivity; electrochemical devices

\section{Introduction to Bio-Based Polymers}

Bio-based polymers are growing in importance over the past few decades due to their potential as replacements or alternatives to conventional polymers. They are the key feature to solve many international issues such as global warming, price fluctuations, the shortage of petroleum resources, pollution, and other economic and ecological issues. In general, bio-based polymers refer to a type of polymer that is produced naturally by living organisms [1]. In other words, it is also called a natural polymer. This type of polymer has the following characteristics: Using natural raw materials as base materials, non-toxic, biodegradable, and sustainable.

Bio-based polymers can be classified into three main categories based on their synthesis and origin of source. The first refers to polymers directly extracted from biomass, such as starch, cellulose, chitosan, and alginates. They are the most abundant and a major resource of bio-based polymers. The second category concerns polymers synthesized from bio-derived monomers, and the third includes polymers synthesized by microorganisms/bacteria. Figure 1 illustrates the classification of bio-based polymers with examples [2]. 


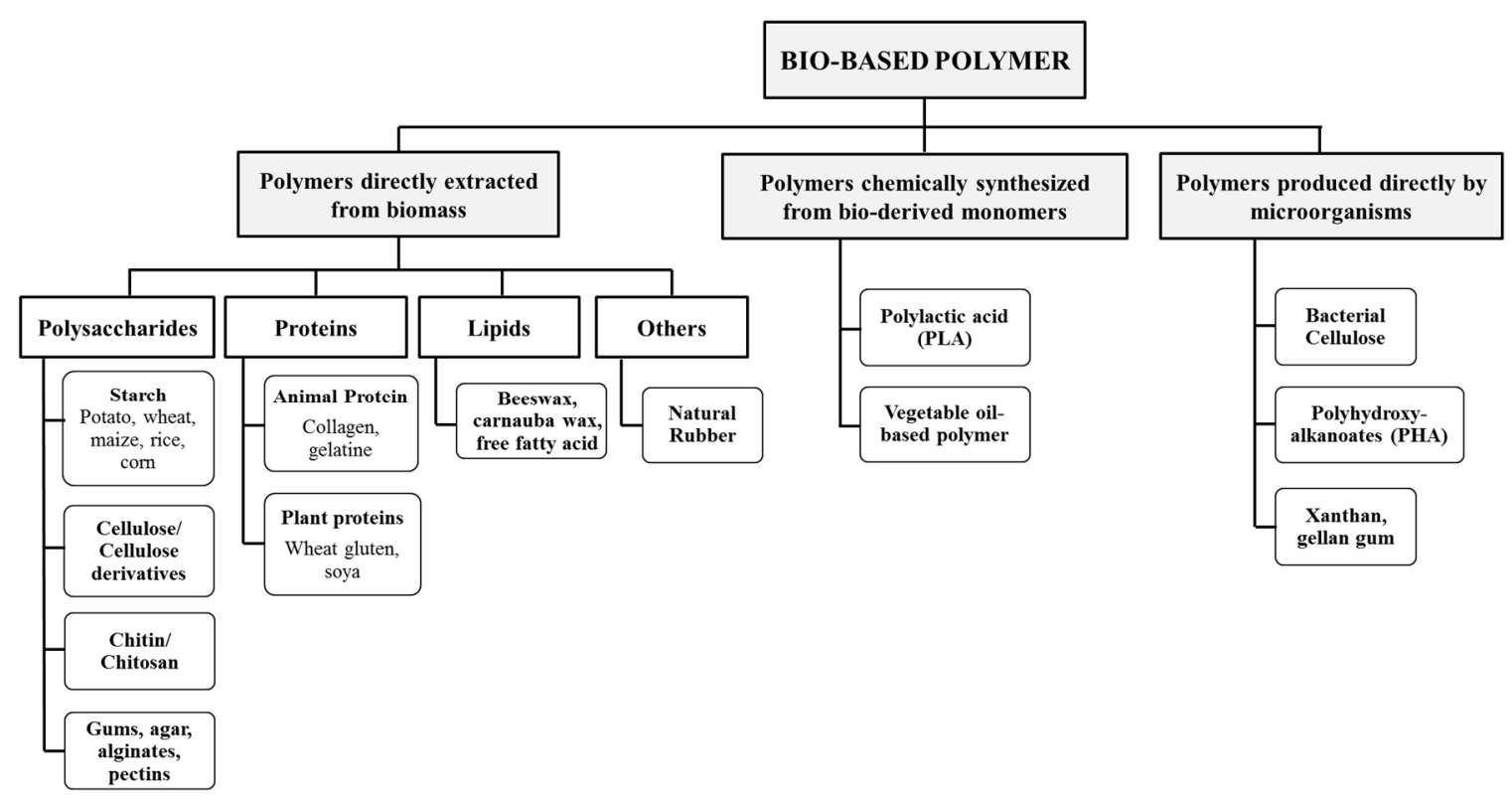

Figure 1. Classification of bio-based polymer (adapted from Malhotra et al. [2]).

The development and innovation of said materials are important, as they hold great potential for research studies and industrial applications. Table 1 presents the most common bio-based polymers, their sources, and related industrial applications. Many studies and development strategies have been devised to discover and to optimize the potential uses of this type of polymer for commercial applications, including the food packaging industry, agricultural purposes, cosmetics, the medical industry, and the pharmaceutical industry. This review offers an overview of bio-based polymers and their applications within the polymer electrolyte field.

Table 1. Various types of bio-based polymers, their sources, and uses [3].

\begin{tabular}{|c|c|c|}
\hline Bio-Based Polymer & Source & Uses \\
\hline Starch & Sago, corn, tapioca, potato, rice & $\begin{array}{l}\text { Adhesives, thickener and stabilizer in foods, } \\
\text { and bio-plastics }\end{array}$ \\
\hline Cellulose & Plants, bacterial & Paper, textile, and wood manufacturing \\
\hline Chitin/chitosan & Shrimp, crab, lobster, shell fish & Cosmetics, foods, pharmaceutics \\
\hline Oils & Palm oil, castor oil, soybean oil, canola oil & Resins, coatings, and adhesives \\
\hline Pectin & Citrus fruits & Additives in food industry, pharmaceutics \\
\hline Latex & Rubber tree, guayule shrubs & Medical, adhesives \\
\hline
\end{tabular}

\section{Insights to the Polymer Electrolyte}

An electrolyte is a significant element in developing electrochemical devices. In general, an electrolyte functions as a medium that allows the flow of ions between a cathode and an anode. An electrolyte also functions as an electronic insulator when the devices fail to work. It is essential to have an electrolyte with a sufficiently high value of ionic conductivity, preferably much higher than $10^{-4} \mathrm{~S} / \mathrm{cm}$ [4]. The electrolyte can be a liquid, a gel, or in a solid form. Although the liquid electrolyte still dominates in many applications, it has several limitations such as flammability issues, leaking, reaction with the electrodes, and corrosion. A polymer electrolyte (PE) offers desirable properties to overcome the problems due to the all solid state condition. It is also inherently safer, as there is no flow and corrosion after damage, it has a wider electrochemical and thermal stability range, it is light weight, and has ease of application to electrochemical devices [5]. In fact, continuous efforts are being taken on polymer electrolytes as they have a great potential to be applied in a wide range of electrochemical devices. Figure 2 shows the use of a conventional electrolyte and polymer electrolyte in a typical electrochemical cell [6]. 


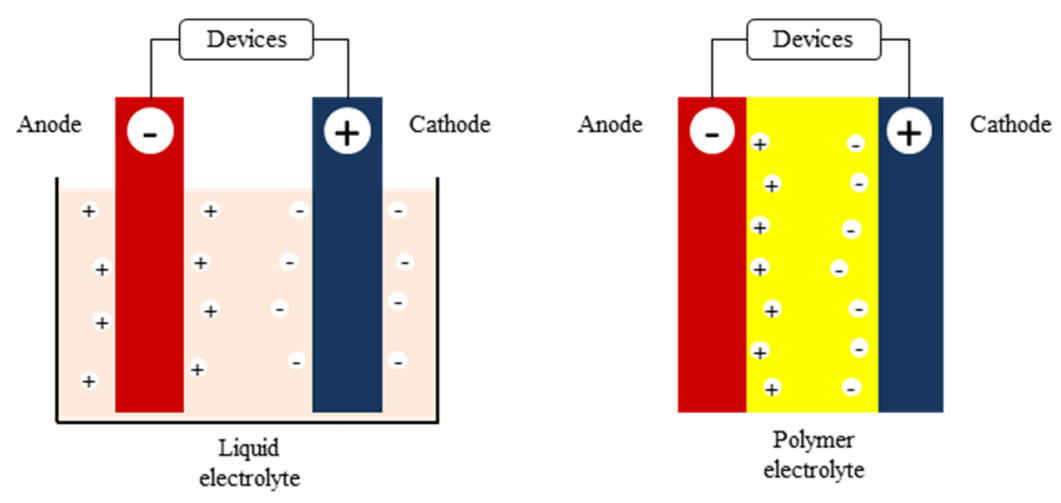

Figure 2. Diagram of a typical electrochemical cell.

Polymer electrolytes (PEs) are a highly specialized multidisciplinary field that cuts across the disciplines of electrochemistry, polymer science, organic chemistry, and inorganic chemistry. In principle, a PE is made up of inorganic salt(s) dispersed in a polymer matrix forming a conducting solid system. The inorganic salt dissociates into ions and contributes to the conductivity. The first discovery of a polymer electrolyte was reported on poly(ethylene oxide) as the polymer host doped with alkali metal ions back in 1973 [7]. The results of the extensive characterization of electrolytes based on poly(ethylene oxide) (PEO), or hosts with similar chemical structures and a great variety of guest salt species, confirmed that many factors, including the choice of electrolyte components, preparative conditions, and thermal history, determine the electrochemical, thermal, and mechanical properties of the electrolyte system. The performance of polymer electrolytes is primarily evaluated based on their ionic conductivity and ion transport properties, which depend on many factors such as the mobility of the polymer chains, the dielectric constant of the polymer host, the degree of salt dissociation and its concentration, as well as the degree of ion aggregation [6].

As for the construction of polymer electrolytes, several factors should be taken into consideration, including the choice of polymer host, the salts/acid dopants (the source of ions), the solvents, and other additives. The polymer host should possess certain characteristics such as good chemical, electrochemical, and photochemical stability, as well as good thermal and mechanical properties. Further, host polymers with a high concentration of polar groups (containing electron donors: $\mathrm{O}, \mathrm{NH}$, $\mathrm{CN}, \mathrm{F}$ ) are preferred. It is important to develop host polymers which have few crystalline phases and a relatively low glass transition temperature. In the amorphous state, greater ionic diffusivity may occur, as ions can move freely due to the low energy barrier. In addition, an amorphous polymer exhibits a flexible backbone that can increase local chain mobility. As a result, the segmental motion of a polymer can enhance the transportation property of the electrolyte [8]. Figure 3 shows the chemical structure of some polar polymers that are widely used as polymer hosts [9].<smiles>COC(C)(C)CCOC(C)(C)C</smiles>

Poly(ethylene oxide)<smiles>COC(=O)C1(C)C2(C)CCCCC1(C)C2</smiles>

Poly(methyl methacrylate)<smiles>CC(C)(C)CC(O)C(C)(C)C</smiles>

Poly(vinyl alcohol)<smiles>CC(C)(CC(C)(C)C(F)(F)F)c1ccccc1</smiles>

Poly(vinyldene fluoride)<smiles>CC(C)(C)CC(Cl)C(C)(C)C</smiles>

Poly(vinyl chloride)

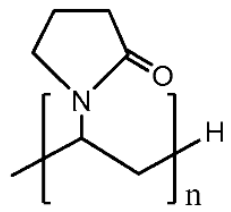

Poly(vinylpyrrolidone)

Figure 3. Chemical structure of some polar polymers used as a host polymer. 
Another crucial aspect is the selection of salts. Salts provide the charge carriers for transportation that generate the conductivity [10]. The most commonly used salts are the salts of alkali metals, alkaline earth metals, and transition metals. The metal cations coordinate with the polar group from the polymer host. The segmental motion of the polymer chains creates free volume into which the ions will migrate and hence create the conductivity. The salts affect the ionic conductivity via several aspects, including complex formation, intramolecular cross-linking of the polymer chains, and the degree of salt dissociation. Apart from a polymer doped with metal salts, several studies of proton conducting electrolytes have been reported. Typically, a polymer is swollen with a solution of proton donors in a polar solvent containing redox sites [11]. As for the electrolyte solvent, it should first satisfy certain criteria. An ideal solvent should be able to dissolve salts to a sufficient concentration, with a high dielectric constant, low vapor pressure, low viscosity so that ion transport can occur, and to be inert to the electrodes.

More than two decades after the introduction of the polymer electrolyte concept, researchers have begun exploring alternatives to conventional polymer hosts derived from petroleum by utilizing bio-based materials as the polymer host. Even though most of the polymer electrolyte theories developed to date are based on synthetic materials, they hold true for bio-based polymers as well. Different types of bio-based polymers, thus, have been explored, such as chitosan, starch, carrageenan, gum, gelatin, natural rubber, and vegetable oil-based polymers. In addition, various electrolyte systems have been investigated with different types of salts, plasticizers, fillers, and ionic liquids. The priority remains the same, which is to produce a polymer electrolyte with a high conductivity, along with good thermal and mechanical properties. The types and characteristics of bio-based polymers, along with the outcomes, are discussed and summarized in the following section.

\section{Bio-Based Polymers Used as Electrolytes}

\subsection{Polymers Extracted from Biomass}

The first category of bio-based polymers is those directly extracted from biomass resources, including polysaccharides, proteins, lipids, and natural rubber. Based on the literature, polymers belonging to the polysaccharide group are the most studied in the polymer electrolyte field. They are easily accessible, widely available, and abundant. It is a fact that plants are the most important producers of polysaccharides. This section discusses the polysaccharides, proteins, and natural rubber-based polymer electrolytes.

\subsubsection{Starch}

Extensive studies have probed starch on account of its rich variety, biodegradability, availability, and abundance in nature. Starch is the end-product of photosynthesis in plants. It is a natural carbohydrate-based polymer that is mainly harvested from corn, potato, wheat, tapioca, and rice [10]. The application of starch does not stop in major food products, but has been extended to various diversified areas. Starch is used in other applications as binders, adhesives, absorbents, and encapsulants [12]. Natural starch is a mixture of linear amylose or poly $(\alpha-1,4-\mathrm{D}-$ glucopyranose $)$ and $\alpha-1,6$-branched amylopectin, wherein their components might varied depending on the plant sources [12,13]. Figure 4 illustrates the structures of amylose and amylopectin. Since these two compounds contain hydroxyl groups, the starch-based polymer emerges as a viable option to be used as a polymer host for electrolyte purposes.

Various types of starch have been investigated for polymer electrolyte studies, such as corn starch, cassava starch, arrowroot starch, sago starch, potato starch, rice starch, and tapioca starch. The starch has also been blended with other polymers such as PEO, chitosan, poly(styrene sulphonic acid), PVA, and methyl cellulose. The initial study concerning starch-based electrolyte was reported by Pawlicka et al. in 2002 for a corn starch/LiClO $/$ /glycerol system [14]. Aside from glycerol, other types of plasticizers have been used, such as glucose, sorbitol, urea, formamide, glutaraldehyde, and ethylene 
carbonate. Additionally, various types of salts have been used as well. The effect of ionic liquid inclusion on the ionic conductivity of a polymer electrolyte has also been investigated. Inorganic fillers, such as silicon dioxide, barium titanate, and graphene oxide, have been studied.

(a)

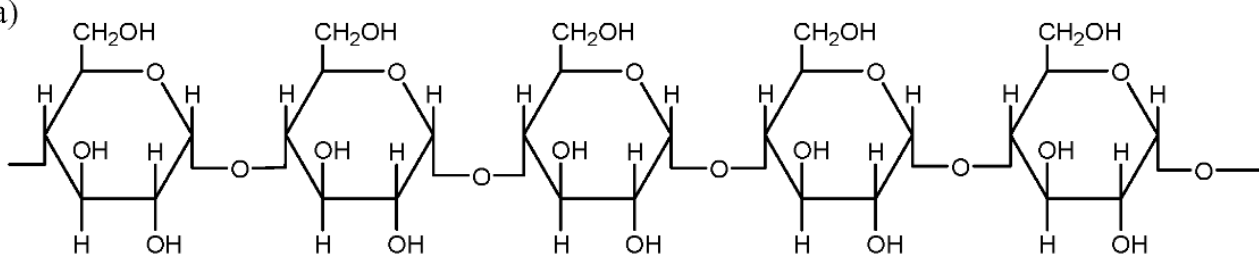

Amylose

(b)
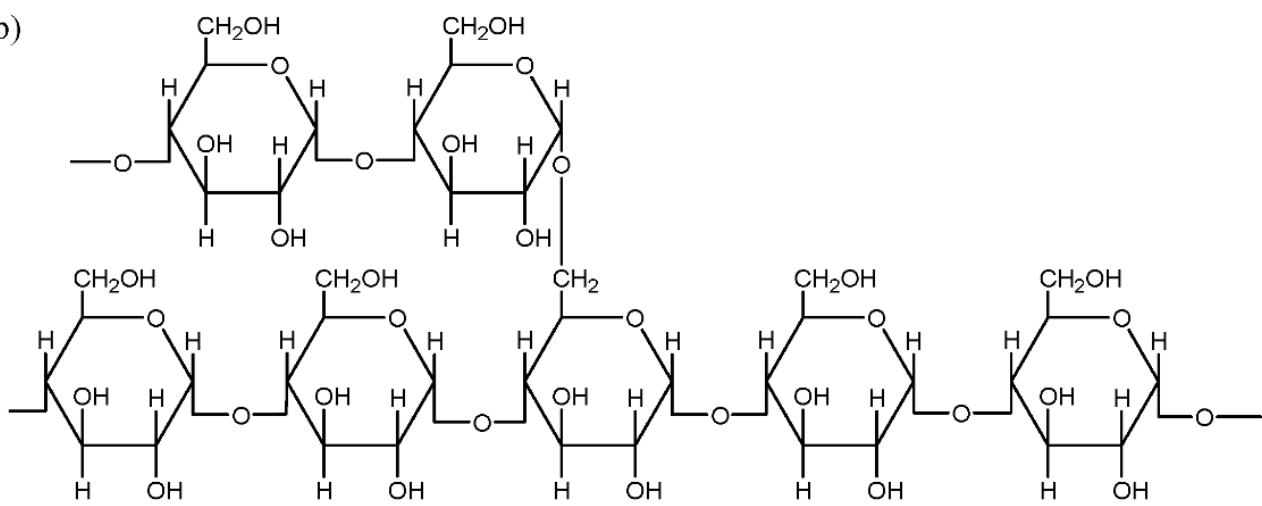

Amylopectin

Figure 4. Representative units of starch (a) amylose and (b) amylopectin.

\subsubsection{Cellulose and Cellulose Derivatives}

Cellulose is by far the most abundant and highly important renewable material on earth. It is the basic component of plant cell walls that has structural and skeletal functions. Cellulose has a high molecular weight and contains a linear homopolysaccharide polymer that consists of $\beta$-D-glucopyranose units in the ${ }^{4} C_{1}$ confirmation joined by $(1 \rightarrow 4)$ glycosidic linkage. The repeating element is made up by two anhydroglucose units. Cellulose exists in the form of microfibrils with a helical organization that contains crystalline and amorphous regions. The proportion of these regions varies depending on the microscopic level of the fiber assembly [15]. Cellulose derivatives can be formed by partially or totally reacting the three hydroxyl groups present in the anhydroglucose unit with various reagents. In fact, many types of cellulose derivatives have been studied, such as methyl cellulose, ethyl cellulose, hydroxyethyl cellulose, hydroxypropyl cellulose, cellulose acetate, cellulose triacetate, cellulose acetate butyrate, hydroxypropyl methyl cellulose, and carboxymethyl cellulose. Figure 5 shows the structures of general cellulose and cellulose derivatives.

Cellulose and cellulose derivatives have been widely applied in numerous applications. They are used as membranes for separation, as binders for drugs, a film coating agent, barrier films, textile applications, and many others [2]. The function of cellulose and cellulose derivatives as hosts in a polymer electrolyte system has been reported by many researchers. By far, cellulose and its derivatives, as presented in Figure 5, have been studied with respect to polymer electrolyte. The initial study was reported in 2001 for hydroxyethyl cellulose. Following that, research was actively conducted by incorporating various types of cellulose derivatives, along with the addition of salts, ionic liquids, plasticizers, and inorganic fillers. 


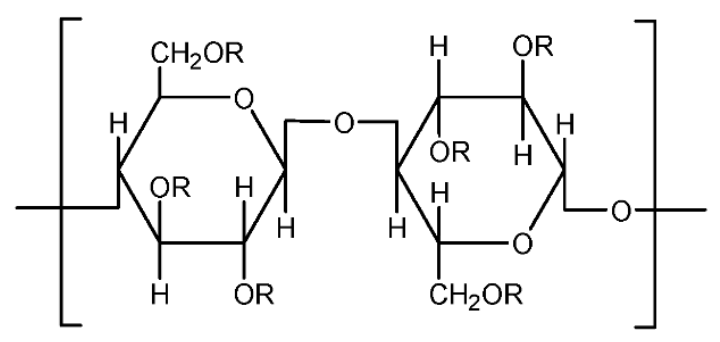

Cellulose

Methyl cellulose (MC)

Ethyl cellulose (EtC)

Hydroxyethyl cellulose (HEC)

Hydroxypropyl cellulose (HPC)

Cellulose acetate (CA)

Cellulose triacetate (CTA)

Cellulose acetate butyrate (CAB)

Hydroxypropyl methyl cellulose (HPMC)

Carboxymethyl cellulose (CMC)

$$
\begin{aligned}
& \mathrm{R}=\mathrm{H} \\
& \mathrm{R}=\mathrm{H} \text { or } \mathrm{CH}_{3} \\
& \mathrm{R}=\mathrm{H} \text { or } \mathrm{CH}_{2} \mathrm{CH}_{3} \\
& \mathrm{R}=\mathrm{H} \text { or } \mathrm{CH}_{2} \mathrm{CH}_{2} \mathrm{OH} \\
& \mathrm{R}=\mathrm{H} \text { or } \mathrm{CH}_{2} \mathrm{CH}(\mathrm{OH}) \mathrm{CH}_{3} \\
& \mathrm{R}=\mathrm{H} \text { or } \mathrm{COCH}_{3} \\
& R=\mathrm{COCH}_{3} \\
& R=\mathrm{H} \text { or } \mathrm{COCH}_{3} \text { or } \mathrm{COCH}_{2} \mathrm{CH}_{2} \mathrm{CH}_{3} \\
& R=\mathrm{H} \text { or } \mathrm{CH}_{3} \text { or } \mathrm{CH}_{2} \mathrm{CH}(\mathrm{OH}) \mathrm{CH}_{3} \\
& R=\mathrm{H} \text { or } \mathrm{CH}_{2} \mathrm{OH}_{2} \mathrm{COONa}
\end{aligned}
$$

Figure 5. Representative units of cellulose and cellulose derivatives.

\subsubsection{Chitosan}

Chitosan has received considerable attention in the polymer field. It shows many interesting properties, such as being non-toxic, biodegradable, and biocompatible. Chitosan consists of 1,4 linked-2-deoxy-2-aminoglucose, which is generated from the deacetylation reaction of chitin. Chitin refers to a natural polysaccharide that can be found in various fungi and the exoskeleton of arthropods [16] such as shrimps, crabs, and lobsters. Figure 6 shows the molecular structure segment of chitin and chitin deacetylation to generate chitosan [17].

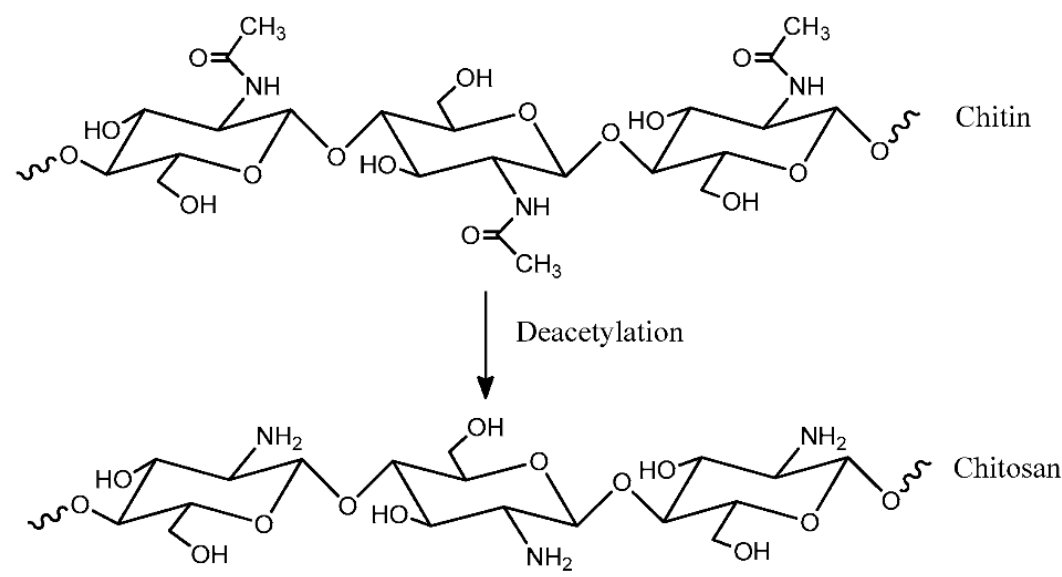

Figure 6. Molecular structure segment of chitin and chitosan.

The application of chitosan has been investigated in the medical field [18], water treatment studies [19], and in food packaging materials [20], to name a few. Interestingly, this polymer can also be applied in polymer electrolyte applications. Chitosan has several polar groups, such as hydroxyl and amino groups, that can act as donors and form complexes with inorganic salts [16]. Chitosan is an amorphous polymer and its glass transition temperature is reported to be $\sim 20{ }^{\circ} \mathrm{C}$ [21]. Owing to these criteria, chitosan may serve as a polymer host for salt solvation. In fact, chitosan is the first biopolymer 
that has been studied for polymer electrolyte applications, reported in 1995. By far, chitosan is also the most widely studied biopolymer for this purpose. The literature portrays that the conductivity of native chitosan without salt is obtained at approximately $10^{-9} \mathrm{~S} / \mathrm{cm}$ [22]. Other types of modified chitosan that have been studied are acetylated chitosan, chitosan acetate, oxipropylated chitosan, hexanoyl chitosan, carboxymethyl chitosan, N-phthaloyl chitosan, sulfonated chitosan, lauroyl chitosan, phosphorylated chitosan, and N-Succinyl chitosan. A wide variety of salts and acid dopants have been applied to improvise the ionic conductivity of the electrolyte system. The incorporation of ionic liquids began in 2010 and many more following that. Several studies have investigated the conductivity of chitosan doped with different types of salts, ionic liquids, plasticizers, and fillers.

\subsubsection{Gum}

Gums are materials classified under polysaccharides with high commercial importance. They are present in many plant, animal, marine, and microbial sources [23]. Gums are available as raw powders. Table 2 lists the sources of common gums and their overall structure. The physiochemical properties of gums are determined by the chemical nature and the molecule shapes. All gums have one common similarity, which is the ability to thicken water and aqueous systems, though the rheological properties of the systems might vary depending on the types of gums being used [24]. Gums have gained many applications in various fields. For instance, in the food industry, gums are used as thickening, emulsifying, and gelling agents. In addition, they have also been applied as adhesives, binders, flocculants, and clarification aids [3]. In this section, gums that originate from marine algae and higher plants are discussed, while gums obtained from microorganisms are described in the latter part of this review.

Table 2. Sources of common gums and their overall structure.

\begin{tabular}{llll}
\hline \multicolumn{2}{c}{ Source } & \multicolumn{1}{c}{ Gum } & \multicolumn{1}{c}{ Structure } \\
\hline Marine algae & & Agar, alginates, carrageenan & Linear, un-branched molecules \\
\hline \multirow{2}{*}{ Higher plants } & Extracts & Pectin & Linear, un-branched molecules \\
& Seeds & Guar gum & Linear molecules with short branches \\
& Exudates & Gum Arabic & Branch-on-branch molecules \\
\hline Microorganism & & Gellan, Xanthan & Linear molecules with short branches \\
\hline
\end{tabular}

Agar

Agar is a hydrocolloid material that is naturally obtained from the extraction of red seaweed, made up of a mixture of two polysaccharides, which are agarose and agaropectin. Agarose is a linear polymer and the predominant component of agar that makes up $70 \%$ of the mixture composed of the agarobiose repeating unit. Agarobiose refers to a disaccharide made up of D-galactose and 3,6-anhydro-L-galactopyranose [25]. Although both compounds share a similar galactose-based backbone, agaropectin contains acidic side groups, such as sulphate and pyruvate, whereas agarose has a neutral charge [26]. Figure 7 shows the representative structures of agarose and agaropectin [25]. The presence of numerous oxygen atoms in the agar structure satisfies the requirement of being a polymer host for an electrolyte, as it can be the possible site for complexation to take place.

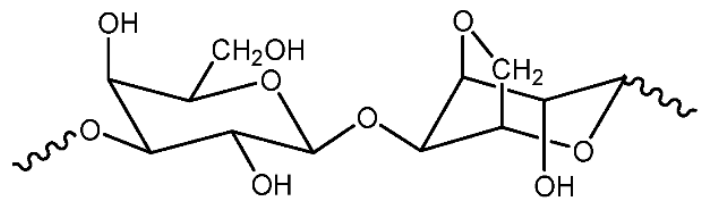

Agarose

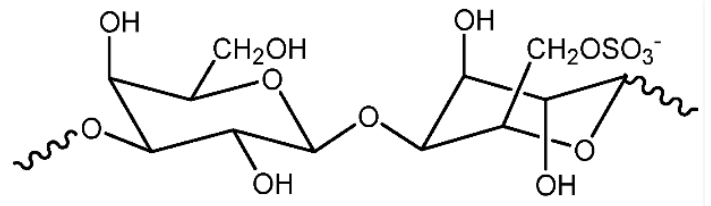

Agaropectin

Figure 7. Representative structure of agarose and agaropectin. 
The early use of agar in electrochemical studies was limited to the preparation of salt bridges in developing a reference electrode. In 2005, Kasem et al. looked into the suitability of agar as a polymer electrolyte host. The study investigated the electrochemical behavior of the electron redox system by using an agar/ $\mathrm{KCl}$ gel electrolyte [27]. Since then, a few studies have examined agar-based electrolytes, which were prepared either in solid or gel form. Although agar is not as extensively studied as compared to cellulose or chitosan, various types of salts as an ion conductor and weak acids as a proton conductor have been investigated. The inclusion of ionic liquids, plasticizers, and nanoparticle fillers has been evaluated as well. So far, the highest room temperature ionic conductivity was achieved at $10^{-3} \mathrm{~S} / \mathrm{cm}$ for an agar-based electrolyte, which is comparable to a liquid electrolyte. The performance of the agar-based electrolyte has been tested for DSSC, ECD, and fuel cell applications.

\section{Carrageenan}

Carrageenan is a linear sulphated polysaccharide polymer extracted from a type of marine red seaweed called Rhodophyceae. It can be classified into three main types: (a) Kappa ( $\mathrm{k}$ )-carrageenan that possesses one sulphate per disaccharide, (b) iota ( $($ )-carrageenan with two sulphates per disaccharide, and (c) lambda ( $\lambda$ )-carrageenan with three sulphates per disaccharide. It has galactose repeating units and 3,6-anhydrogalactose, both sulphated and non-sulphated, amalgamated by alternating $\alpha-(1,3)$ and $\beta-(1,4)$ glycosidic links. Figure 8 portrays the representative units of carrageenan [28]. This polymer has been used extensively in the food, cosmetic, and pharmaceutical industries [22]. Studies pertaining to PE have, so far, looked into kappa and iota carrageenan, while none have researched lambda carrageenan.
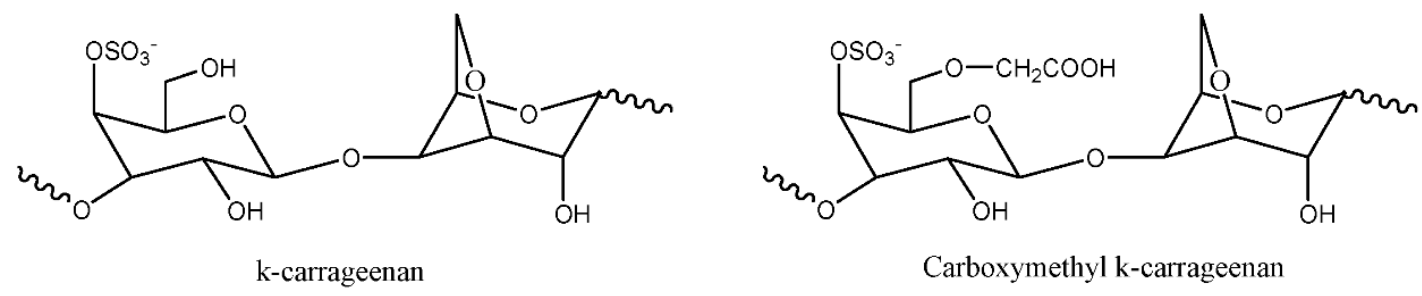

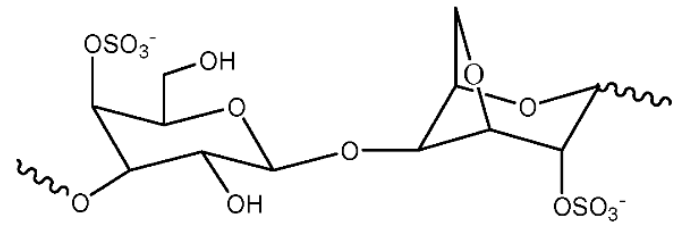

i-carrageenan

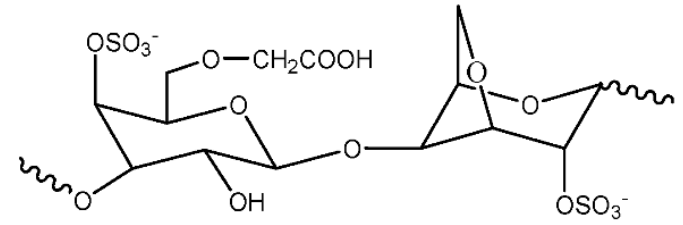

Carboxymethyl i-carrageenan

Figure 8. Representative units of k-carrageenan, i-carrageenan, CMC k-carrageenan, and CMC i-carrageenan.

The initial study concerning the potential of carrageenan in the polymer electrolyte field was reported by Mobarak et al. in 2012 [29]. The team prepared k-carrageenan and a carboxymethyl (CMC) $\mathrm{k}$-carrageenan-based electrolyte via a solution casting method with a $1 \%(v / v)$ aqueous acetic acid solution. The room temperature ionic conductivity of $5.34 \times 10^{-7} \mathrm{~S} / \mathrm{cm}$ was achieved for $\mathrm{k}$-carrageenan. Interestingly, upon the modification to $\mathrm{CMC} \mathrm{K}$-carrageenan, the conductivity increased three magnitudes to $2.02 \times 10^{-4} \mathrm{~S} / \mathrm{cm}$. This enhancement was attributed to the fact that modification increased the amount of oxygen in the system, hence providing greater vacancies for protons or cations to coordinate. Another study was conducted by the same team utilizing CMC K-carrageenan and l-carrageenan with different ratios of lithium nitrate $\left(\mathrm{LiNO}_{3}\right)$ salts $(5-30 \mathrm{wt} \%)$. The highest ionic conductivity for $\mathrm{CMC}$ $\mathrm{K}$-carrageenan was obtained at $30 \mathrm{wt} \% \mathrm{LiNO}_{3}$ salt with $5.85 \times 10^{-3} \mathrm{~S} / \mathrm{cm}$, while the best conductivity of $\mathrm{t}$-carrageenan was recorded at $5.51 \times 10^{-3} \mathrm{~S} / \mathrm{cm}$ at $20 \mathrm{wt} \%$ of salt [22]. Another study concerning 
K-carrageenan was conducted by Rudziah et al. In their study, carboxymethyl k-carrageenan (CMKC) was blended with carboxymethyl cellulose (CMC). The cellulose was extracted from kenaf fiber and modified to produce $\mathrm{CMC}$. The films of $\mathrm{CMKC} / \mathrm{CMC}$ blend were prepared via the solution casting method with various ratios. They explained that the increase in conductivity was related with the increase in segmental motion and the fraction of the amorphous region [30]. The carrageenan-based electrolyte has been tested for various applications, such as DSSC, ECD, super-capacitors, and fuel cells.

Pectin

Pectin is one type of polymer that is not widely explored in the polymer electrolyte field. It is a natural polymer, present naturally in the cell walls of terrestrial plants, and is abundant in vegetables and fruits. Citrus fruits, such as oranges and limes, contain substantial amounts of pectin. At present, the major sources are citrus peels and apple pomace, which are the by-products from the extraction of citrus and apple juices [31]. Typically, pectin is used in food products as it has beneficial effects upon the health of the consumer. Commercial pectin exists as a white to light brown powder. Pectin is usually applied as a gelling agent for food production. Chemically, pectin is a complex polysaccharide that is composed mainly of D-galacturonic acid resides in $\alpha-(1-4)$ chain $(65 \mathrm{wt} \%)$. Pectin is also a group of substances which forms gel when dissolved in water under suitable conditions [32]. Figure 9 shows the representative unit of pectin [33].

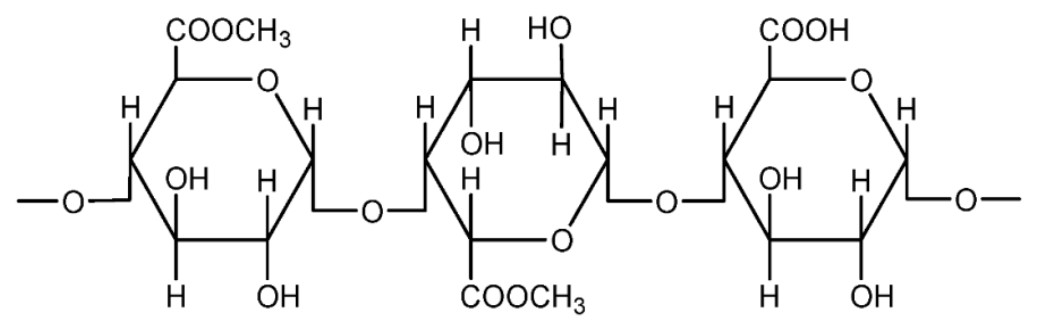

Figure 9. Representative unit of pectin.

The study of pectin-based polymer electrolytes was initiated in 2009 by Andrande et al. Pectin was doped with a fixed amount of $\mathrm{LiClO}_{4}$ and plasticized with glycerol for $0-70 \mathrm{wt} \%$. The outcome reflected a good transparency feature with the pectin-based electrolyte film, which would be hardly achievable by a solid electrolyte. The best ionic conductivity value of $4.7 \times 10^{-4} \mathrm{~S} / \mathrm{cm}$ was obtained for the sample plasticized with $68 \mathrm{wt} \%$ of glycerol [33].

\section{Guar gum and Gum Arabic}

Guar gum is processed from the endosperm of seeds from the cluster bean, Cyamopsis tetragonolobus, which belongs to the Leguminosae family. It contains a complex of polysaccharides called galactomannan, which is made up of D-galactose and D-mannose [34]. Figure 10 illustrates the representative unit of Guar gum [35]. This polymer contains an abundance of hydroxyl groups and tends to form hydrogen bonds when added to water. It is mainly used as an additive in food, pharmaceuticals, paper, textiles, and the cosmetics industry [36]. The application of guar gum as a polymer electrolyte began in 2014 initiated by Sudhakar et al. They prepared a solid polymer electrolyte of Guar gum $/ \mathrm{LiClO}_{4} /$ glycerol system and obtained a high room temperature ionic conductivity of $2.2 \times 10^{-3} \mathrm{~S} / \mathrm{cm}$ [37]. Meanwhile, the effects of the addition of ionic liquid and filler were studied by other researchers $[35,38]$.

Gum Arabic, which is also known as Acacia gum, refers to the tree gum exudate of the Acacia tree. It is a highly branched polysaccharide and contains glycoprotein components. Gum Arabic is abundantly available and primarily used as an emulsifier, stabilizer, and thickening agent. The study of gum Arabic was performed by Khalid and Hartono for a supercapacitor application. They prepared a gel-like electrolyte by mixing gum Arabic with ortho-phosphoric acid. The gel electrolyte demonstrated excellent conductivity and supercapacitive performance. 


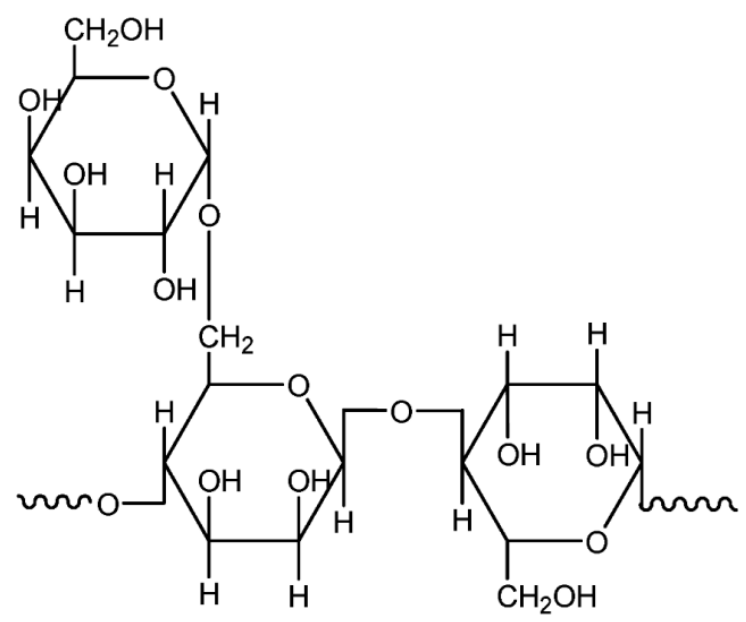

Figure 10. Representative unit of Guar gum.

\subsubsection{Gelatin}

Gelatin is a soluble protein substance derived from collagen, a natural protein present in bonds, cartilage, and skin. The main source of gelatin is from bovine and porcine animals, but it also can be extracted from fish and poultry. Gelatin properties are influenced by several factors, such as the source, animal age, and collagen type. It has widespread applications, for example, emulsifiers, foaming agents, biodegradable packaging materials, and colloid stabilizers [39]. Gelatin is a polydisperse protein that is composed of a mixture of different chain types with varying molecular weights [40]. Figure 11 shows a representative unit of gelatin [41]. The study of gelatin-based polymer electrolytes started way back in 2007 by Diogo F et al [42]. The solid polymer electrolyte system was made up of gelatin/glycerol/acetic acid with the best room temperature ionic conductivity achieved at $10^{-5} \mathrm{~S} / \mathrm{cm}$. Subsequently, various types of salts have been investigated. The electrolyte system has also been tested for EDC and DSSC.<smiles>CC(NC(=O)NCCC(=O)N1CCCC1C(=O)NC(CCCNC(=N)N)C(=O)NCC(=O)NC(CCC(=O)O)C(=O)N1CC(O)CC1C(=O)O)C(=O)O</smiles>

Figure 11. Representative unit of gelatin.

\subsubsection{Natural Rubber}

Natural rubber is an elastic polymer obtained from the latex of rubber trees, made up of thousands of repeating isoprene units $\left(\mathrm{C}_{5} \mathrm{H}_{8}\right)_{\mathrm{n}}$. Natural rubber is produced from thousands of different plant species. In industrial applications, the most important source of natural rubber derives from the Hevea brasiliensis tree, which is mostly planted in the South-East Asia and Western Africa regions. Natural rubber has several distinctive properties, such as a low glass transition temperature, as well as good elasticity and adhesion characteristics. It has been widely applied in various applications, from household goods to the high end automotive industries [43]. Nonetheless, in a polymer electrolyte system, the natural rubber itself does not have any polar group in the structure to facilitate the ion mobility. Thus, modified natural rubber such as epoxidized natural rubber (ENR), ENR-25 and ENR-50 
(where 25 and 50 represent the epoxy groups), as well as poly(methyl methacrylate)-grafted natural rubber (MG), MG-30 and MG-49 (where 30 and 49 indicate the percentages of methyl methacrylate grafted to the natural rubber), as shown in Figure 12, have been introduced to overcome the shortcoming.
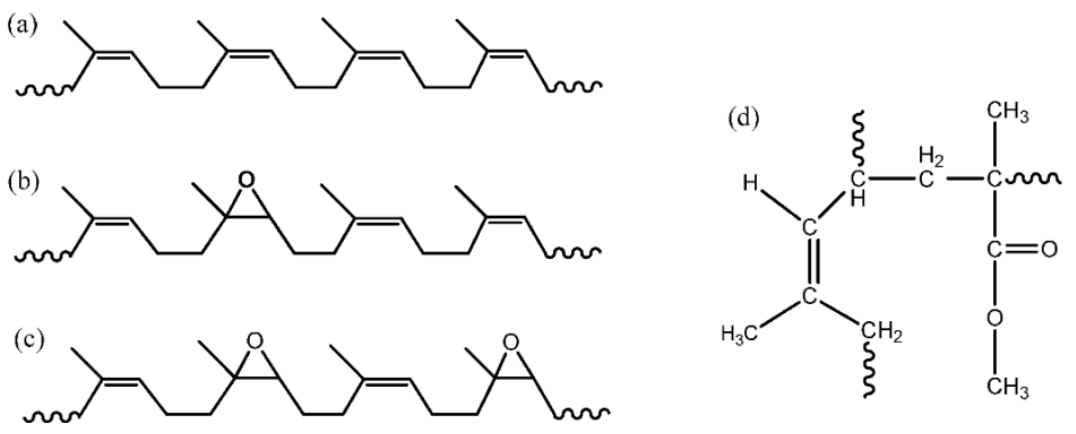

Figure 12. Structure of (a) natural rubber, (b) ENR-25, (c) ENR-50, and (d) MG-30 and MG-49.

The study of natural rubber-based polymer electrolytes was initiated by Yoshizawa et al. [44] in the year 2000. They blended natural rubber with polyethylene oxide via a solution casting method to produce a solid film electrolyte. The room temperature ionic conductivity obtained was $10^{-6} \mathrm{~S} / \mathrm{cm}$. Consequently, the focus shifted to natural rubber derivatives such as MG-30, MG-49, ENR-25, and ENR-50. Derivatives MG-30 and MG-49 were prepared by polymerizing a methyl methacrylate monomer in latex so that the polymer chains become attached to the rubber molecules. Meanwhile, ENR can be chemically modified from natural rubber, wherein some of the unsaturated group is converted into epoxide groups and randomly distributed [45]. Apart from being used as a single polymer host, natural rubber and its derivatives are blended with other polymers to enhance the properties of the electrolyte system.

\subsection{Polymers Chemically Synthesized from Bio-Derived Monomers}

The second type of bio-based polymer refers to polymers that are chemically synthesized from naturally-derived monomers. It is practically possible to produce tailor-made polymers with highly versatile properties using monomers. Nevertheless, studies pertaining to this type of polymer are only limited to poly(lactic acid) and some vegetable oil-based polyurethanes.

\subsubsection{Poly(lactic acid)}

Poly(lactic acid) (PLA) is a linear aliphatic thermoplastic biodegradable polyester derived from two major pathways, namely, ring opening of lactide or direct polycondensation of lactic acid, a monomeric precursor obtained from renewable resources. The monomer is produced by a fermentation process of sugar feedstock, such as dextrose or chemical synthesis. Sugar feedstock can be obtained either directly from sources (sugar cane, sugar beet) or through conversion of starch from corn, potato, wheat, rice, or agricultural wastes. Figure 13 presents the general structure of poly (lactic acid).

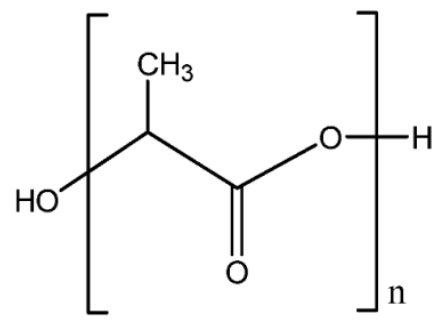

Figure 13. General structure of polylactic acid. 
Several studies have elaborately reported on PLA electrolytes. In a study, a PLA-based electrolyte was prepared via the solution casting method [46]. The outcomes showed that the conductivity of pure PLA at room temperature was $9.46 \times 10^{-12} \mathrm{~S} / \mathrm{cm}$. Ethylene carbonate was added as the plasticizing agent. The incorporation of $\mathrm{LiClO}_{4}$ salt as the main ion carrier into the PLA/EC system enhanced the conductivity to $1.44 \times 10^{-6} \mathrm{~S} / \mathrm{cm}$. The addition of $\mathrm{SiO}_{2}$ as a ceramic filler further enhanced the conductivity of the PLA electrolyte system to $1.29 \times 10^{-5} \mathrm{~S} / \mathrm{cm}$. Subsequently, Chew [47] improvised a PLA-based electrolyte by incorporating aluminum oxide $\left(\mathrm{Al}_{2} \mathrm{O}_{3}\right)$ as another type of ceramic filler. A similar composition was prepared as the previous $\mathrm{PLA} / \mathrm{EC} / \mathrm{LiClO}_{4}$ system and looked into various fillers. Based on the output, the inclusion of $4 \% \mathrm{Al}_{2} \mathrm{O}_{3}$ displayed the highest conductivity at $2.07 \times 10^{-5} \mathrm{~S} / \mathrm{cm}$. Osinska-Broniarz et al. [48] investigated the performance of PLA blended with poly 3-hydroxybutyrate (PHB) as a gel polymer electrolyte. The PLA/PHB blend polymer was prepared via the electrospinning method. The PLA/PHB membrane was then soaked in an electrolyte that consisted of lithium hexafluorophosphate $\left(\mathrm{LiPF}_{6}\right)$ in a mixture of ethylene carbonate and dimethyl carbonate. A maximum room temperature ionic conductivity of $1.5 \times 10^{-5} \mathrm{~S} / \mathrm{cm}$ was obtained at a 70:30 weight ratio of PLA to PHB. In another study, a ternary polymer electrolyte based on PLA, an ionic liquid (Pyr 14 TFSI), and LiTFSI salt was prepared by Osada et al. [49] The materials were mixed by using the hot-pressed technique and melted together without including any solvents. The PLA/Pyr ${ }_{14}$ TFSI/LiTFSI system achieved a conductivity of $2.1 \times 10^{-4} \mathrm{~S} / \mathrm{cm}$ at $60^{\circ} \mathrm{C}$.

\subsubsection{Vegetable Oil-Based Polyurethane}

Vegetable oils are an excellent alternative to petrochemical feedstock. They can be used as a reliable starting material to produce new polymers. Vegetable oils are derived from plant sources, thus they are natural, abundant, and renewable. They can be classified into edible and non-edible oils. The most common vegetable oils include soybean oil, palm oil, sunflower oil, rapeseed oil, jatropha oil, and castor oil, to name a few. Vegetable oils are made up of long carbon chains and the main constituent is known as triglycerides. Some oils contain carbon-to-carbon double bonds (unsaturation site) that can be converted into the desired functional groups through chemical synthesis. For the polymer electrolyte purpose, palm oil, jatropha oil, and castor oil have been used as the raw material to prepare polyurethane (PU). Figure 14 shows the reaction of a vegetable oil-based polyol with the isocyanate group to produce polyurethane [50].

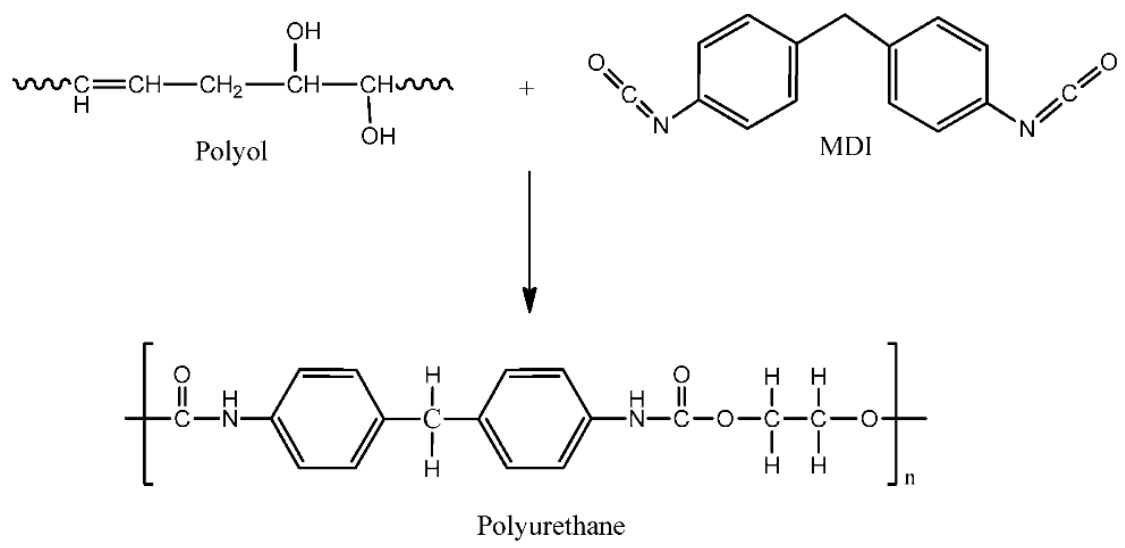

Figure 14. The reaction of polyol and MDI to produce polyurethane.

Su'ait et al. prepared palm oil-based polyurethane as a solid polymer electrolyte. In their study, palm kernel oil polyol (PKO-p) was reacted with 2,4'-methylene diphenyl diisocyanate (2,4'MDI) to produce PU. The electrolyte was prepared via the solution casting method with the inclusion of lithium iodide as the dopant salt at $10-30 \mathrm{wt} \%$ concentration, with ethylene carbonate as the plasticizing agent at a fixed amount of $20 \mathrm{wt} \%$. The best room temperature ionic conductivity of $7.6 \times 10^{-4} \mathrm{~S} / \mathrm{cm}$ was obtained at $25 \mathrm{wt} \%$ LiI salt [51]. Another study of palm-based PU electrolyte was conducted by 
Daud et al., in which PKO-p was mixed with 2,4'-MDI in acetone at room temperature. The effect of various concentrations of $\mathrm{LiCF}_{3} \mathrm{SO}_{3}$ salt was evaluated. The highest room temperature conductivity was $1.6 \times 10^{-5} \mathrm{~S} / \mathrm{cm}$ obtained at $30 \mathrm{wt} \% \mathrm{LiCF}_{3} \mathrm{SO}_{3}[8]$.

Castor oil-based polyurethane was synthesized by Salmiah et al. Castor oil is a great alternative to be used in generating PU as it is a non-edible oil. In their study, castor oil polyol was reacted with 4,4'-diphenylmethane diisocyanate (MDI) to produce PU. The electrolyte was prepared by mixing PU with lithium iodide (LiI) and sodium iodide (NaI) salt from $0-40 \mathrm{wt} \%$ salt concentration. The maximum ionic conductivity at room temperature was recorded at $30 \mathrm{wt} \%$ for both salts, with values $1.78 \times 10^{-6}$ and $4.28 \times 10^{-7} \mathrm{~S} / \mathrm{cm}$ for LiI and NaI, respectively. They suggested that the PU/LiI system exhibited higher conductivity, when compared to PU/NaI due to the smaller cation size of $\mathrm{Li}^{+}$than $\mathrm{Na}^{+}$. The sizes of $\mathrm{Li}^{+}$and $\mathrm{Na}^{+}$cations were 0.76 and $1.02 \AA$, respectively. Theoretically, a smaller cation size generates higher conductivity due to the higher mobility of the cation [52].

Jatropha oil is another example of a non-edible oil that has been used to prepare polyol for polyurethane production. Mustapa et al. prepared a solid polymer electrolyte from jatropha oil-based polyurethane doped with $\mathrm{LiClO}_{4}$ and ethylene carbonate. The highest conductivity of $1.29 \times 10^{-4} \mathrm{~S} / \mathrm{cm}$ was achieved at $25 \mathrm{wt} \%$ of $\mathrm{LiClO}_{4}$ salt.

\subsection{Polymers Produced by Microorganisms}

The direct production of bio-based polymers can be achieved by using microorganisms or genetically modified bacteria. In fact, a wide range of bio-based polymers with material properties suitable for industrial applications can and have been synthesized. This section will discuss some of the polymers that have been investigated as a host polymer electrolyte.

\subsubsection{Bacterial Cellulose}

Bacterial cellulose is mainly used in the food industry, as well as in biomedical and cosmeceutical applications. It is produced by acetic acid bacteria in synthetic and non-synthetic media via oxidative fermentation [53]. Some cellulose-producing bacteria are Acetobacter, Rhizobium, Agrobacterium, and Sarcina. One important cellulose-producing bacterium is Acetobacter xylinum, which is the most efficient synthesizer of bacterial cellulose. The chemical structure of bacterial cellulose is similar to that of plant cellulose, except for the physical and chemical properties [54]. Bacteria cellulose possesses unique characteristics over plant cellulose, such as (1) absence of lignin and hemicellulose, (2) high degree of polymerization, and (3) extremely high water-holding capacity and excellent biodegradability. Despite this, the current price of this bacterial cellulose is still considered as too high for certain applications. The study of bacterial cellulose polymer electrolytes only started in 2015, and since then, only two studies have been reported $[55,56]$. Both studies successfully recorded high ionic conductivity similar to that of liquid electrolytes.

\subsubsection{Gellan Gum and Xanthan Gum}

Gellan gum is an extracellular, anionic polysaccharide made up of tetrasaccharides that consist of two glucoses, namely, one glucuronic acid and one rhamnose ring. It is produced from the fermentation of Sphingomonas elodea (ATCC 31461) by inoculating a fermentation medium with the microorganism. It is available in two forms, specifically high acyl (native gellan) and low acyl [57]. While the low acyl gellan is a firm, brittle, and non-elastic gel, the high acyl gellan on the other hand is a soft, elastic, and non-brittle gel. The gellan gum is mainly studied in the ophthalmology field for lenses with drug delivery properties [58]. One important property of gellan gum is the high thermal stability that can reach up to $120^{\circ} \mathrm{C}$ and its thermal reversibility [59]. Figure 15 illustrates the representative units of gellan gum [58]. Gellan gum has been investigated as a polymer host doped with various types of salts and acid dopants. The initial study, undertaken in 2012, looked into applications for electrochemical devices. 


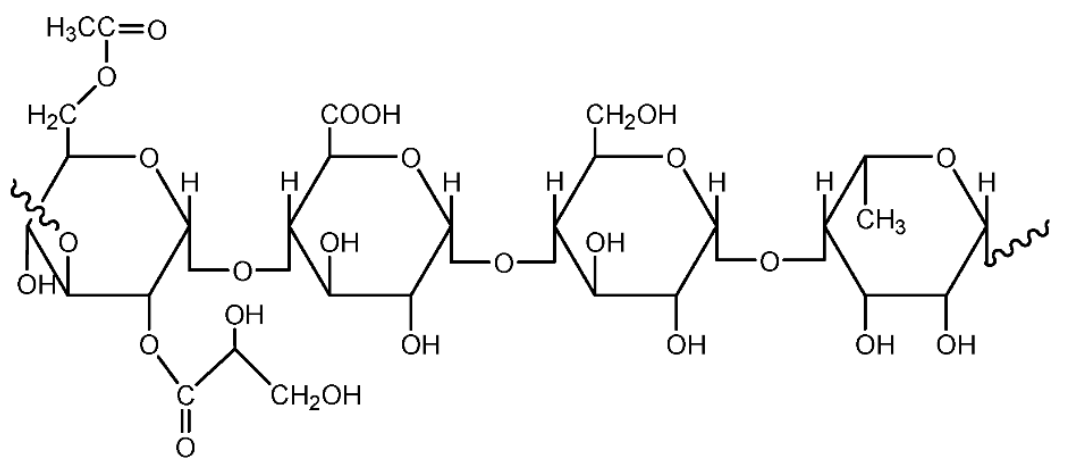

Figure 15. Representative unit of Gellan Gum.

Xanthan gum is a high molecular weight polysaccharide produced via the microbiological fermentation of sugar cane/corn by the microorganism Xanthomonas campestris. Xanthan gum has excellent thermal stability, as well as good solubility and stability, thus it is suitable for use in the food, cosmetic, and pharmaceutical industries. Xanthan is an acidic polymer with the shape of a five-fold helix, made up of pentasaccharide subunits to form a cellulose backbone with trisaccharide side-chains composed of mannose $(\beta-1,4)$ glucuronic acid, $(\beta-1,2)$ mannose attached to alternate glucose residues in the backbone by $\alpha-1,3$ linkages [23,60]. Figure 16 portrays the representative units of xanthan gum [61]. Only two studies have been reported regarding xanthan gum-based electrolytes. This particular electrolyte system displayed exceptional conductivity after being tested for dye sensitized solar cell and supercapacitor applications $[23,61]$.

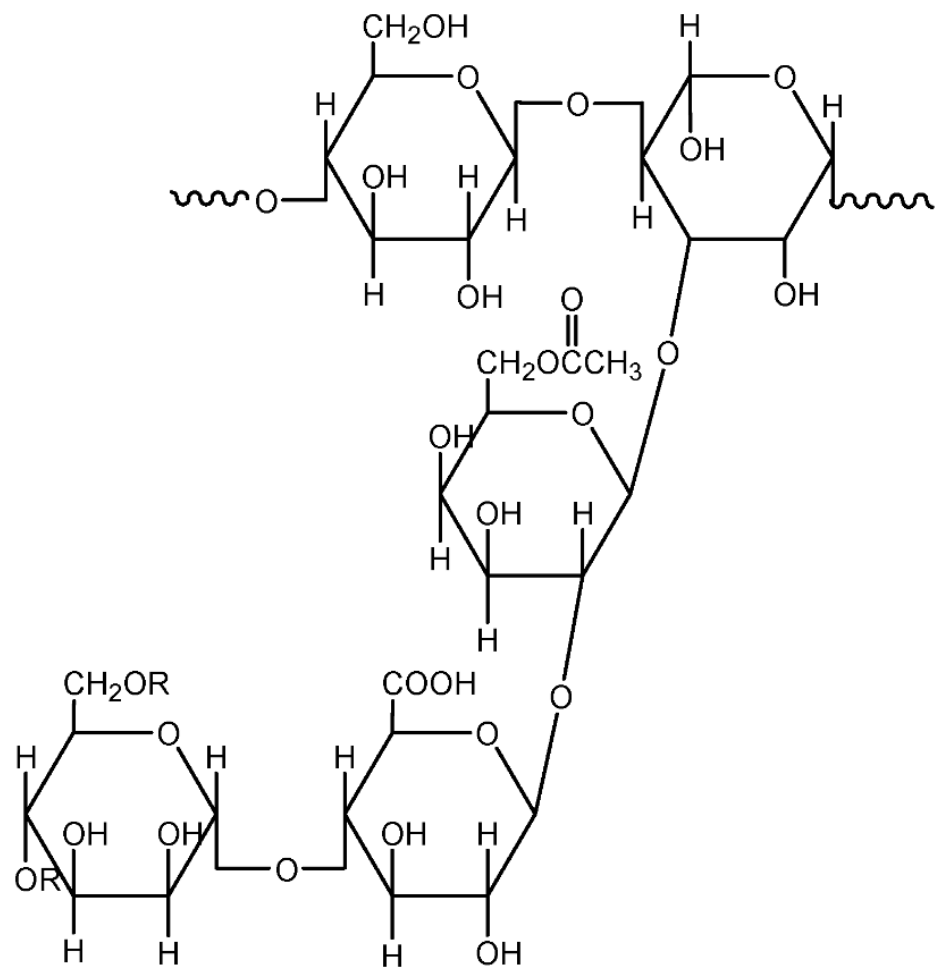

Figure 16. Representative unit of xanthan gum.

\subsection{Development of Bio-Based Polymer Electrolyte}

The use of bio-based polymers in the polymer electrolyte field is not a new concept. In fact, they have been used for a long time; however, only in the past three decades have they been extensively investigated in this field. Generally, each of the bio-based polymer hosts studied has its advantages and limitations. The advantages of these polymers are obvious, including the renewability, availability, 
and environmentally friendly nature. In spite of that, they have some shortcomings in terms of economic and technical aspects. The cost of said materials are relatively higher than the conventional petroleum-based polymers. Some technical issues like hydrophilic character and poor mechanical properties have hampered their applications. Thus, the properties must be improved in order for such electrolytes to be applied commercially. The main challenge is to produce an electrolyte system with high conductivity, whilst maintaining the electrochemical, thermal, and mechanical properties. Efforts have been taken to achieve this objective via several approaches, such as blending the polymer with other compatible polymers, the incorporation of fillers and the addition of plasticizers. Blending techniques aim to obtain new and unique materials with additional properties without sacrificing their original properties [62]. In general, polymer blend refers to mixture of at least two substances, polymers or copolymers, where the ingredient content is above $2 \mathrm{wt} \%$. This method is applied because of its simple preparation and its ease of control over physical properties by compositional change [63].

Meanwhile, the addition of a plasticizer could improve the conductivity of a polymer electrolyte by reducing the glass transition temperature that facilitates the mobility of ions within the medium and dissociating ion aggregates [16]. Further, a high value of the dielectric constant of a plasticizer could solvate more salt, thereby increasing the number of free mobile ions [64]. The incorporation of organic/inorganic fillers could reduce the crystallinity and enhance both the mechanical and electrochemical properties. It has been discovered that the conductivity is strongly dependent on the particle size and concentration of the filler. Small-sized particles at low quantities are favorable and promote an enhanced conductivity [65]. In addition, room temperature ionic liquids (RTIL) have garnered interest as a substitute to organic solvents. RTIL is a molten salt containing bulky and asymmetric organic cations and contains highly delocalized charge inorganic anions. RTIL has some interesting features, such as excellent thermal and chemical stabilities, relatively high ionic conductivity, non-volatile, non-flammable, and wider electrochemical potential window [10].

The collective data of prior finding on bio-based polymer electrolytes using different types of bio-based polymer are shown in Table 3. The result was classified based on their source, origin, application, physical, and electrochemical properties. Various systems have been investigated by introducing different types of polymer hosts, salts, plasticizers, fillers, and ionic liquids. By far, starch, cellulose, and chitosan are the most widely studied and reported bio-based material in the polymer electrolyte field. The trend shows that the choice of salt used commonly depends on the intended end-use application. Different types of plasticizer have been used to enhance the ionic conductivity, such as glycerol, glucose, sorbitol, urea, formamide, glutaraldehyde, ethylene carbonate, propylene carbonate, and so on. Nano-oxide materials are a common choice to be used as fillers. Meanwhile, a wide variety of ionic liquids has been explored. From the findings, it is possible for the bio-based polymer electrolyte to achieve a room temperature ionic conductivity of $10^{-2}$ to $10^{-3} \mathrm{~S} \mathrm{~cm}^{-1}$, which is similar to the conductivity of the liquid electrolyte. Besides, gel electrolytes show better performance in comparison to the solid type. For a deep understanding, a complete test on electrochemical, thermal, and physical properties of the electrolyte is necessary to conduct in order to improve their performance in actual applications. Therefore, for the purpose of reliable electrochemical device applications, the bio-based polymer electrolytes should possess these characteristics: (1) Ionic conductivity $\left(\geq 10^{-4} \mathrm{~S} \mathrm{~cm}^{-1}\right)$; (2) high ionic transference number $\left(t_{\text {ion }} \sim 1\right)$; $(3)$ high chemical, thermal, and electrochemical stability; (4) good mechanical strength; and (5) good compatibility with the electrodes. 
Table 3. Summary of prior study on bio-based polymer electrolytes.

\begin{tabular}{|c|c|c|c|c|c|c|c|c|c|c|}
\hline \multirow{2}{*}{ Polymer } & \multirow{2}{*}{ Electrolyte System } & \multirow{2}{*}{ State } & \multicolumn{4}{|c|}{ Electrochemical Properties } & \multicolumn{2}{|c|}{ Physical Properties } & \multirow{2}{*}{ Device } & \multirow{2}{*}{ Ref } \\
\hline & & & $\sigma(\mathrm{s} / \mathrm{cm})$ & I-TN & Stability (V) & $E_{a}$ & $\mathrm{~T}_{\mathrm{g}}\left({ }^{\circ} \mathrm{C}\right)$ & Structural & & \\
\hline \multirow{47}{*}{ Starch } & Corn starch-LiClO 4 -glycerol & Solid & $7.9 \times 10^{-5}$ & - & - & - & -58 & Amorph & - & [14] \\
\hline & Corn starch-LiClO 4 -glycerol & Solid & $5.0 \times 10^{-5}$ & - & - & - & - & - & - & [66] \\
\hline & Corn starch-LiClO 4 -glycerol & Gel & $10^{-4}$ & - & - & $0.35 \mathrm{eV}$ & - & - & - & [67] \\
\hline & Corn starch-NaCl-glucose & & - & - & - & - & - & - & - & \\
\hline & Corn starch-NaCl-glycerol & & - & - & - & - & - & - & - & \\
\hline & Corn starch-NaCl-sorbitol & Solid & - & - & - & - & - & - & - & [68] \\
\hline & Corn starch-NaCl-urea & & - & - & - & - & - & - & - & \\
\hline & Corn starch-NaCl-formamide & & $10^{-3}$ & - & - & - & - & - & - & \\
\hline & 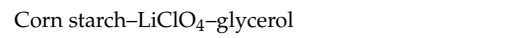 & & $6.1 \times 10^{-5}$ & - & - & - & - & - & - & {$[69]$} \\
\hline & Cassava starch- $-\mathrm{LiClO}_{4}$-glycerol & Solid & $8.4 \times 10^{-5}$ & - & - & - & - & - & - & [69] \\
\hline & Starch- $\mathrm{NH}_{4} \mathrm{NO}_{3}$ & Solid & $2.8 \times 10^{-5}$ & - & - & $0.41 \mathrm{eV}$ & - & - & - & [12] \\
\hline & Corn starch-- $\mathrm{LiClO}_{4}-$ glycerol & Solid & $1.1 \times 10^{-4}$ & - & - & - & -75 & - & - & [70] \\
\hline & Corn starch-LiTFSI-AmIm][Cl] & Solid & $4.2 \times 10^{-4}$ & - & - & - & - & - & - & [71] \\
\hline & Corn starch-LiPF $\left.-\left[\mathrm{BmIm}_{6}\right] \mathrm{PF}_{6}\right]$ & Solid & $1.5 \times 10^{-4}$ & - & - & - & - & - & - & [72] \\
\hline & Arrowroot starch-NaI-glutaraldehyde & Solid & $6.7 \times 10^{-4}$ & 0.95 & - & - & - & - & - & [73] \\
\hline & Tapioca starch/PEO-NH $\mathrm{NH}_{4} \mathrm{NO}_{3}$ & Solid & $2.8 \times 10^{-7}$ & - & - & - & - & Semi-cr & - & [63] \\
\hline & Corn starch-LiPF ${ }_{6}-[\mathrm{BmIm}][\mathrm{Tf}]$ & Solid & $6.0 \times 10^{-4}$ & - & - & $0.01 \mathrm{eV}$ & -29 & Amorph & - & [74] \\
\hline & Sago starch- $-\mathrm{NH}_{4} \mathrm{Br}$ & Solid & $6.9 \times 10^{-9}$ & - & - & $0.07 \mathrm{eV}$ & - & - & - & [75] \\
\hline & Corn starch- $\mathrm{LiClO}_{4}-\mathrm{SiO}_{2}$ & Solid & $1.2 \times 10^{-4}$ & - & - & - & 87.1 & - & - & [76] \\
\hline & Corn starch-LiTFSI-[AmIm][Cl] & Gel & $5.7 \times 10^{-2}$ & - & - & $4.8 \mathrm{~kJ} / \mathrm{mol}$ & - & - & - & [77] \\
\hline & Corn starch-LiTFSI-DES & Solid & $1.0 \times 10^{-3}$ & - & - & - & - & - & - & [78] \\
\hline & Potato starch-NH $\mathrm{NH}_{4} \mathrm{I}$ & Solid & $2.4 \times 10^{-4}$ & 0.95 & - & - & - & - & - & [13] \\
\hline & Starch/chitosan-LiClO ${ }_{4}$-glycerol & Solid & $3.7 \times 10^{-4}$ & - & - & $0.52 \mathrm{eV}$ & - & - & - & [79] \\
\hline & Corn starch- $\mathrm{AgNO}_{3}$ & Solid & - & - & - & $0.71 \mathrm{eV}$ & - & Amorph & - & [80] \\
\hline & Corn starch-Lil-glycerol & Solid & $9.6 \times 10^{-4}$ & - & - & $0.16 \mathrm{eV}$ & - & Amorph & - & [81] \\
\hline & Corn starch- $-\mathrm{AgNO}_{3}$ & Solid & $1.0 \times 10^{-9}$ & - & - & - & - & - & - & [82] \\
\hline & Rice starch-LiI & Solid & $4.7 \times 10^{-5}$ & - & - & $0.41 \mathrm{eV}$ & - & - & - & [83] \\
\hline & Poly(styrene sulphonic acid)/starch-LiClO ${ }_{4}$-glycerol & Solid & $5.7 \times 10^{-3}$ & - & - & - & - & - & - & [84] \\
\hline & Potato starch-NaI-glutaraldehyde-PEG & Solid & $1.8 \times 10^{-4}$ & 0.99 & - & - & 75 & - & - & [85] \\
\hline & Corn starch-LiOAc-glycerol & Solid & $1.0 \times 10^{-3}$ & - & 2.1 & $0.14 \mathrm{eV}$ & - & Amorph & - & [86] \\
\hline & Sago starch-KI- $\mathrm{I}_{2}$ & Solid & $3.4 \times 10^{-4}$ & - & - & - & - & - & - & [87] \\
\hline & Rice starch-LiI-MPII- $\mathrm{TiO}_{2}$ & Solid & $3.6 \times 10^{-4}$ & - & - & $0.22 \mathrm{eV}$ & - & Amorph & DSSC & [88] \\
\hline & Corn starch-LiPF 6 - $\left[\mathrm{BmIm}^{2}\right]\left[\mathrm{PF}_{6}\right]$ & & $1.5 \times 10^{-4}$ & - & 2.9 & - & - & - & & [89] \\
\hline & Corn starch-LiPF $-[\mathrm{BmIm}][\mathrm{Tf}]$ & Solid & $3.2 \times 10^{-4}$ & - & 3.1 & - & - & - & Super-capacitor & {$[89]$} \\
\hline & Corn starch- $\mathrm{LiClO}_{4}$ & Solid & $1.6 \times 10^{-6}$ & - & - & $0.64 \mathrm{eV}$ & 64 & - & - & [90] \\
\hline & Corn starch/chitosan- $\mathrm{NH}_{4} \mathrm{I}$-glycerol & Solid & $1.3 \times 10^{-3}$ & 0.99 & 1.9 & $0.18 \mathrm{eV}$ & - & - & - & [91] \\
\hline & Starch/chitosan- $-\mathrm{NH}_{4} \mathrm{I}$ & Solid & $3.0 \times 10^{-4}$ & - & - & $0.20 \mathrm{eV}$ & - & Amorph & - & [92] \\
\hline & Starch/chitosan- $-\mathrm{NH}_{4} \mathrm{Cl}$-glycerol & Solid & $5.1 \times 10^{-4}$ & - & - & $0.19 \mathrm{eV}$ & -0.37 & - & - & [93] \\
\hline & Starch/chitosan- $\mathrm{NH}_{4} \mathrm{Br}-\mathrm{EC}$ & Solid & $1.4 \times 10^{-3}$ & 0.92 & 1.8 & $0.17 \mathrm{eV}$ & - & Amorph & EDLC & [94] \\
\hline & Corn starch- $\mathrm{LiClO}_{4}-\mathrm{SiO}_{2}$ & Solid & $1.2 \times 10^{-4}$ & - & 3.0 & $0.25 \mathrm{eV}$ & - & - & EDLC & [95] \\
\hline & Rice starch-LiI & & $4.7 \times 10^{-5}$ & - & - & - & -12 & Amorph & & \\
\hline & Rice starch-NH $\mathrm{NH}_{4} \mathrm{I}$ & Solid & $1.4 \times 10^{-4}$ & - & - & - & -38 & Amorph & DSSC & [96] \\
\hline & Rice starch-NaI & & $4.8 \times 10^{-4}$ & - & - & - & -42 & Amorph & & \\
\hline & Rice starch-NaI-MPII & Solid & $1.2 \times 10^{-3}$ & - & - & - & -58 & Amorph & DSSC & [97] \\
\hline & Corn starch-NH $\mathrm{NH}_{4} \mathrm{Br}$-glycerol & Solid & $1.8 \times 10^{-3}$ & 0.98 & 1.6 & $0.11 \mathrm{eV}$ & - & Amorph & - & [98] \\
\hline & Corn starch-LiPF $\left.6-\left[\mathrm{BmIm}_{[}\right] \mathrm{PF}_{6}\right]$ & Solid & $2.0 \times 10^{-4}$ & - & 2.9 & - & - & Amorph & - & [99] \\
\hline & Potato starch/PVA-KCl-glycerol & Solid & $5.4 \times 10^{-5}$ & 0.97 & - & $0.12 \mathrm{eV}$ & - & Amorph & - & [100] \\
\hline
\end{tabular}


Table 3. Cont

\begin{tabular}{|c|c|c|c|c|c|c|c|c|c|c|}
\hline \multirow{2}{*}{ Polymer } & \multirow{2}{*}{ Electrolyte System } & \multirow{2}{*}{ State } & \multicolumn{4}{|c|}{ Electrochemical Properties } & \multicolumn{2}{|c|}{ Physical Properties } & \multirow{2}{*}{ Device } & \multirow{2}{*}{ Ref. } \\
\hline & & & $\sigma(\mathrm{s} / \mathrm{cm})$ & I-TN & Stability (V) & $E_{a}$ & $\mathrm{~T}_{\mathrm{g}}\left({ }^{\circ} \mathrm{C}\right)$ & Structural & & \\
\hline & Potato starch/chitosan- $-\mathrm{LiCF}_{3} \mathrm{SO}_{3}$ & Solid & $7.1 \times 10^{-7}$ & - & - & - & - & - & - & [101] \\
\hline & Potato starch/chitosan- $\mathrm{LiCF}_{3} \mathrm{SO}_{3}$-glycerol & Solid & $1.3 \times 10^{-3}$ & - & - & $0.11 \mathrm{eV}$ & - & Amorph & - & [102] \\
\hline & Corn starch- $-\mathrm{LiClO}_{4}-\mathrm{BaTiO}_{3}$ & Solid & $1.8 \times 10^{-4}$ & - & 3.1 & - & 17.2 & - & EDLC & [103] \\
\hline & Corn starch-LiTFSI & Solid & $3.4 \times 10^{-4}$ & - & - & - & - & - & Battery & [104] \\
\hline & Potato starch- $\mathrm{Mg}\left(\mathrm{C}_{2} \mathrm{H}_{3} \mathrm{O}_{2}\right)_{2}-[\mathrm{BmIm}][\mathrm{Cl}]-$ glycerol & Solid & $1.1 \times 10^{-5}$ & 0.92 & - & - & - & - & - & [105] \\
\hline & Potato starch/Poly(vinyl alcohol)-LiBr-glycerol & Solid & $10^{-3}$ & - & - & - & - & - & - & [106] \\
\hline & Potato starch/methylcellulose-- $\mathrm{LiClO}_{4}$-glycerol & Solid & $4.3 \times 10^{-4}$ & - & - & - & - & Amorph & - & [107] \\
\hline & Starch/PVA-NH 4 SCN & Solid & $1.3 \times 10^{-4}$ & - & - & - & - & Amorph & - & [108] \\
\hline & Tapioca starch/chitosan- $\mathrm{NH}_{4} \mathrm{NO}_{3}-[\mathrm{EmIm}]\left[\mathrm{NO}_{3}\right]$ & Solid & $7.4 \times 10^{-5}$ & - & - & - & - & - & - & [109] \\
\hline & Starch-NaCl & Gel & $6.2 \times 10^{-2}$ & - & - & - & - & - & - & [110] \\
\hline & Corn starch- $\mathrm{LiClO}_{4}$-glycerol & Solid & $9.0 \times 10^{-3}$ & - & - & - & - & Amorph & - & [111] \\
\hline & Potato starch-LiCF $3 \mathrm{SO}_{3}-[\mathrm{BmIm}][\mathrm{Cl}]-\mathrm{GO}$ & Solid & $4.8 \times 10^{-4}$ & - & - & - & - & Amorph & - & [112] \\
\hline & Corn starch- $\mathrm{NaClO}_{4}$-glutaraldehyde & Solid & $10^{-2}$ & - & 2.4 & - & - & - & Super-capacitor & [113] \\
\hline & Potato starch-NADES & Solid & $2.9 \times 10^{-3}$ & - & - & - & - & Amorph & - & [114] \\
\hline & Potato starch/methyl cellulose- $\mathrm{NH}_{4} \mathrm{NO}_{3}$-glycerol & Solid & $1.3 \times 10^{-3}$ & 0.98 & 1.8 & - & -27.5 & Amorph & EDLC & {$[115,116]$} \\
\hline \multirow{30}{*}{ Cellulose } & HEC/DPEO-LiClO 4 & Solid & $2.1 \times 10^{-5}$ & - & - & $0.17 \mathrm{eV}$ & - & - & - & {$[117,118]$} \\
\hline & HEC-LiClO 4 -glycerol & Solid & $9.5 \times 10^{-5}$ & - & - & - & -60 & Amorph & - & [14] \\
\hline & Cellulose $/ \mathrm{PEO}-\mathrm{LiCF}_{3} \mathrm{SO}_{3}$ & Solid & $10^{-7}$ & - & - & $53 \mathrm{~kJ} / \mathrm{mol}$ & - & - & - & [119] \\
\hline & $\mathrm{HPC} / \mathrm{PEO}-\mathrm{LiCF}_{3} \mathrm{SO}_{3}-\mathrm{PC}$ & Gel & $10^{-3}$ & - & - & $16 \mathrm{~kJ} / \mathrm{mol}$ & - & - & - & [120] \\
\hline & EO-EPI/nano-cellulose-LiClO & Solid & $1.6 \times 10^{-4}$ & - & - & - & - & - & - & [121] \\
\hline & POE/nano-cellulose-LiTFSI-TEGDME & Solid & $10^{-6}$ & - & - & - & - & - & - & [122] \\
\hline & $\mathrm{HPC} /$ Jeffamine- $-\mathrm{LiClO}_{4}$ & Solid & $1.3 \times 10^{-5}$ & - & - & - & - & - & - & [123] \\
\hline & POE/nano-cellulose-LiTFSI & Solid & $10^{-7}$ & - & - & - & - & - & - & {$[124,125]$} \\
\hline & Cellulose acetate- $\mathrm{LiClO}_{4}$ & Solid & $4.9 \times 10^{-3}$ & - & - & - & - & - & Super-capacitor & [126] \\
\hline & Ethyl cellulose- $-\mathrm{LiClO}_{4}-\mathrm{PC}$ & Gel & $6.5 \times 10^{-3}$ & - & - & $0.18 \mathrm{eV}$ & - & - & - & [127] \\
\hline & PVDF-HPF/cellulose-LiPF 6 -EC/DMC & Solid & $4.4 \times 10^{-3}$ & - & 4.8 & - & - & Semi-cr & - & [128] \\
\hline & Cellulose acetate- $-\mathrm{NH}_{4} \mathrm{BF}_{4}-\mathrm{SiO}_{2}$ & Gel & $7.9 \times 10^{-3}$ & - & - & - & - & - & Battery & [129] \\
\hline & Cellulose triacetate-LiTFSI-Pyr ${ }_{1,3}$ TFSI & Gel & $10^{-4}$ & - & - & - & - & - & - & [130] \\
\hline & Cellulose-acrylic acid-[BmIm][I] & Gel & $7.3 \times 10^{-3}$ & - & - & - & - & - & DSSC & [131] \\
\hline & Cellulose acetate- $\mathrm{NH}_{4} \mathrm{I}-\mathrm{PC}$ & Solid & $1.2 \times 10^{-4}$ & - & - & - & - & - & - & [132] \\
\hline & Cellulose acetate- $-\mathrm{NH}_{4} \mathrm{BF}_{4}-\mathrm{TiO}_{2}$ & Gel & $1.4 \times 10^{-2}$ & - & - & - & - & - & Battery & [133] \\
\hline & Cellulose acetate- $\mathrm{NH}_{4} \mathrm{BF}_{4}-\mathrm{PEG}$ & Solid & $1.4 \times 10^{-5}$ & - & - & - & - & - & - & {$[134-136]$} \\
\hline & CMC-DTAB & Solid & $7.7 \times 10^{-4}$ & 0.92 & - & $0.09 \mathrm{eV}$ & - & Amorph & - & [137] \\
\hline & $\mathrm{PEG} /$ network cellulose- $\mathrm{LiClO}_{4}$ & Gel & $10^{-4}$ & - & 4.7 & - & - & Amorph & - & [138] \\
\hline & CN-HPC-LiI-I $\mathrm{I}_{2}-\mathrm{MHII}$ & Gel & $2.5 \times 10^{-3}$ & - & - & - & - & Amorph & DSSC & [139] \\
\hline & Cellulose acetate-LiTFSI-DES & Gel & $2.6 \times 10^{-3}$ & - & - & $4.23 \mathrm{~kJ} / \mathrm{mol}$ & - & Amorph & - & {$[140,141]$} \\
\hline & Cellulose acetate-LiTFSI-[AmIm][Cl] & Solid & $1.8 \times 10^{-3}$ & - & - & - & - & Amorph & - & [142] \\
\hline & Methyl cellulose- $\mathrm{LiCF}_{3} \mathrm{SO}_{3}$ & Solid & $2.1 \times 10^{-5}$ & - & - & - & - & Amorph & - & [143] \\
\hline & Methyl cellulose-PEG- $\mathrm{NH}_{4} \mathrm{NO}_{3}$ & Solid & $10^{-6}$ & - & 2.4 & - & - & Amorph & EDLC & [144] \\
\hline & Methyl cellulose-KOH-DMC & Solid & $10^{-5}$ & - & - & - & - & - & - & [145] \\
\hline & $\mathrm{PE} / \mathrm{PVDF} /$ Cellulose acetate butyrate- $-\mathrm{LiPF}_{6}-\mathrm{EC} / \mathrm{EMC}$ & Gel & $2.5 \times 10^{-3}$ & - & - & - & - & - & Battery & [146] \\
\hline & PEO/CMC-NaI- $\mathrm{I}_{2}-\mathrm{MPII}$ & Gel & $2.0 \times 10$ & - & - & - & - & - & DSSC & [147] \\
\hline & Cellulose acetate-LiBOB-GBL & Gel & $5.4 \times 10^{-3}$ & - & 4.7 & - & - & - & - & [148] \\
\hline & $\mathrm{PEO} /$ Network cellulose- $-\mathrm{LiClO}_{4}$ & Solid & $8.0 \times 10^{-7}$ & - & 5.0 & - & - & Semi-cr & - & [149] \\
\hline & PVDF-HFP/HPMC-LiPF 6 & Gel & $3.8 \times 10^{-4}$ & - & 5.0 & - & - & Amorph & - & [150] \\
\hline
\end{tabular}


Table 3. Cont

\begin{tabular}{|c|c|c|c|c|c|c|c|c|c|c|}
\hline \multirow{2}{*}{ Polymer } & \multirow{2}{*}{ Electrolyte System } & \multirow{2}{*}{ State } & \multicolumn{4}{|c|}{ Electrochemical Properties } & \multicolumn{2}{|c|}{ Physical Properties } & \multirow{2}{*}{ Device } & \multirow[b]{2}{*}{ Ref. } \\
\hline & & & $\sigma(\mathrm{s} / \mathrm{cm})$ & I-TN & Stability (V) & $E_{a}$ & $\mathrm{~T}_{\mathrm{g}}\left({ }^{\circ} \mathrm{C}\right)$ & Structural & & \\
\hline & Cellulose acetate-LiTFSI-[Amim][Cl] & Solid & $4.7 \times 10^{-2}$ & - & - & $1.25 \mathrm{~kJ} / \mathrm{mol}$ & - & - & - & [151] \\
\hline & $\mathrm{CMC}-\mathrm{LiClO}_{4}-\mathrm{PC}$ & Gel & - & _- & - & - 1.20 & _- & _- & ECD & {$[152]$} \\
\hline & MC-LiBOB & Solid & - & - & - & - & - & - & - & [153] \\
\hline & MFC/BEMA/PEGMA-NaI- $\mathrm{I}_{2}$ & Gel & - & - & - & - & - & Amorph & DSSC & [154] \\
\hline & PVDF/Methyl cellulose-LiPF 6 -EC/EMC & Gel & $2.0 \times 10^{-4}$ & - & - & - & - & - & Battery & [155] \\
\hline & CMC-Citric acid & Solid & $4.4 \times 10^{-7}$ & 0.89 & - & - & - & - & - & [156] \\
\hline & $\mathrm{MG}-49 / \mathrm{CMC}-\mathrm{LiCF}_{3} \mathrm{SO}_{3}$ & Solid & $3.3 \times 10^{-7}$ & - & - & - & - & Amorph & - & [157] \\
\hline & Methyl cellulose-NaI & Solid & $2.7 \times 10^{-5}$ & - & - & - & - & - & - & {$[158,159]$} \\
\hline & MC-NWF-LiPF 6 -EC/DMC/EMC & Gel & $2.9 \times 10^{-4}$ & - & - & - & - & - & Battery & [160] \\
\hline & Cellulose acetate/PVDF-HFP-LiTFSI-TEGDME & Gel & $5.5 \times 10^{-4}$ & - & 4.7 & - & - & - & Battery & [161] \\
\hline & $\mathrm{PEO} / \mathrm{CMC}-\mathrm{NaClO}_{4}$ & Solid & - & - & - & - & - & - & Battery & [162] \\
\hline & Cellulose acetate-LiTFSI & Solid & $5.6 \times 10^{-4}$ & - & - & - & - & Amorph & - & [163] \\
\hline & $\mathrm{HEC}-\mathrm{H}_{3} \mathrm{PO}_{4}$ & Solid & $4.1 \times 10^{-3}$ & - & - & $0.12 \mathrm{eV}$ & - & - & Super-capacitor & [164] \\
\hline & $\mathrm{CMC}-\mathrm{LiPF}_{6}-\mathrm{EC} / \mathrm{DMC} / \mathrm{DEC}$ & Gel & $4.8 \times 10^{-4}$ & - & - & $25.5 \mathrm{~kJ} / \mathrm{mol}$ & - & - & Battery & [165] \\
\hline & 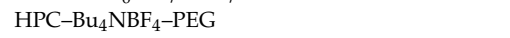 & Gel & $3.5 \times 10^{-5}$ & - & - & - & -37 & - & ECD & [166] \\
\hline & Cellulose acetate- $\mathrm{NH}_{4} \mathrm{I}$ & Solid & $10^{-4}$ & - & - & - & - & - & DSSC & [167] \\
\hline & $\mathrm{CMC}-\mathrm{CH}_{3} \mathrm{COONH}_{4}$-BMATFSI & Solid & $2.2 \times 10^{-3}$ & - & - & $0.06 \mathrm{eV}$ & - & - & - & [168] \\
\hline & HEC-LiPF 6 -EC/DMC/DEC & Gel & $1.8 \times 10^{-4}$ & - & - & $3.57 \mathrm{~kJ} / \mathrm{mol}$ & - & - & Battery & [169] \\
\hline & $\mathrm{CMC}-\mathrm{NH}_{4} \mathrm{Cl}$ & Solid & $1.4 \times 10^{-3}$ & - & - & - & - & - & - & [170] \\
\hline & $\mathrm{CMC}-\left(\mathrm{NH}_{4}\right)_{2} \mathrm{CO}_{3}$ & Solid & $7.7 \times 10^{-6}$ & - & - & - & - & Amorph & - & [171] \\
\hline & $\mathrm{CMC}-\mathrm{NH}_{4} \mathrm{~F}$ & Solid & - & - & - & - & - & Semi-cr & - & [172] \\
\hline & $\mathrm{HPMC}-\mathrm{Mg}\left(\mathrm{CF}_{3} \mathrm{SO}_{3}\right)_{2}-[\mathrm{BmIm}][\mathrm{Tf}]$ & Solid & $2.4 \times 10^{-4}$ & - & - & $1.28 \mathrm{eV}$ & 27.5 & Amorph & - & [173] \\
\hline & CMC-Oleic acid-glycerol & Solid & $1.6 \times 10^{-4}$ & _- & _- & - & - & - & - & {$[174]$} \\
\hline & Cellulose acetate- $\mathrm{LiClO}_{4}-\mathrm{PC}$ & Gel & $5.3 \times 10^{-3}$ & _- & _- & _- & - & - & $\mathrm{ECD}$ & [175] \\
\hline & Cellulose acetate- $-\mathrm{NH}_{4} \mathrm{I}-\mathrm{EC}$ & Solid & $10^{-3}$ & - & - & - & - & Amorph & - & [176] \\
\hline & $\begin{array}{l}\mathrm{PVDF} / \text { cellulose acetate } \\
\text { (n) }\end{array}$ & Gel & $2.9 \times 10^{-3}$ & - & 5.2 & - & - & - & Battery & [177] \\
\hline & $\begin{array}{l}\text { butyrate/PE-LiPF } 6-\mathrm{EC} / \mathrm{DMC} / \mathrm{EMC}-\mathrm{SiO}_{2} \\
\text { Cellulose acetate-LiNO }\end{array}$ & Solid & $1.9 \times 10^{-3}$ & - & 4.1 & $0.16 \mathrm{eV}$ & - & Amorph & ECD & [178] \\
\hline & Cellulose acetate- $-\mathrm{NH}_{4} \mathrm{SCN}$ & Solid & $3.3 \times 10^{-3}$ & 0.99 & - & $0.15 \mathrm{eV}$ & 113.7 & Amorph & Battery & [179] \\
\hline & Cellulose acetate-LiTFSI-BDG & Gel & $2.9 \times 10^{-3}$ & - & 3.8 & - & - & Amorph & - & [180] \\
\hline & Lignocellulose/potato starch-LiPF 6 -EC/DMC/EMC & Gel & $1.3 \times 10^{-3}$ & - & - & $12.7 \mathrm{~kJ} / \mathrm{mol}$ & - & - & Battery & [181] \\
\hline & Lignocellulose-PEG & Gel & $3.2 \times 10^{-3}$ & - & - & - & _- & - & Battery & [182] \\
\hline & PVA/chitosan/CNC-Acetic acid & Solid & $6.4 \times 10^{-4}$ & _- & _- & _- & _- & Amorph & Fuel cell & [183] \\
\hline & $\mathrm{HEC}-\mathrm{Li}_{2} \mathrm{~B}_{4} \mathrm{O}_{7}$-glycerol & Solid & $4.6 \times 10^{-3}$ & - & - & - & - & Amorph & - & [184] \\
\hline & Cellulose acetate- $\mathrm{NH}_{4} \mathrm{NO}_{3}$ & Solid & $1.0 \times 10^{-3}$ & 0.97 & 4.3 & $0.05 \mathrm{eV}$ & 111.6 & Amorph & ECD & [185] \\
\hline & $\mathrm{CMC}-\left(\mathrm{NH}_{4}\right)_{2} \mathrm{CO}_{3}$ & Solid & $7.7 \times 10^{-6}$ & 0.98 & - & $0.21 \mathrm{eV}$ & - & - & - & [186] \\
\hline & Acetylated chitosan- $\mathrm{LiNO}_{3}$ & Solid & $10^{-4}$ & - & - & - & - & Amorph & Battery & [187] \\
\hline
\end{tabular}


Table 3. Cont

\begin{tabular}{|c|c|c|c|c|c|c|c|c|c|c|}
\hline \multirow{2}{*}{ Polymer } & \multirow{2}{*}{ Electrolyte System } & \multirow{2}{*}{ State } & \multicolumn{4}{|c|}{ Electrochemical Properties } & \multicolumn{2}{|c|}{ Physical Properties } & \multirow{2}{*}{ Device } & \multirow[b]{2}{*}{ Ref. } \\
\hline & & & $\sigma(\mathrm{s} / \mathrm{cm})$ & I-TN & Stability (V) & $E_{a}$ & $\mathrm{~T}_{\mathrm{g}}\left({ }^{\circ} \mathrm{C}\right)$ & Structural & & \\
\hline & Chitosan acetate-NaI & Solid & $4.9 \times 10^{-5}$ & - & - & - & - & - & Battery & [188] \\
\hline & Chitosan- $\mathrm{NaClO}_{4}$ & Solid & $4.6 \times 10^{-2}$ & - & - & _- & _- & _- & Battery & [189] \\
\hline & Oxipropylated chitosan/polyether-LiTFSI & Solid & - & - & - & - & - & - & - & [190] \\
\hline & Chitosan acetate- $-\mathrm{LiCF}_{3} \mathrm{SO}_{3}-\mathrm{EC}$ & Solid & $10^{-5}$ & - & - & - & - & - & - & {$[21,191]$} \\
\hline & Chitosan-KCl & Solid & - & - & - & _- & - & - & - & [192] \\
\hline & Chitosan acetate- $-\mathrm{LiCF}_{3} \mathrm{SO}_{3}-\mathrm{EC}$ & Solid & $1.3 \times 10^{-5}$ & - & - & - & - & - & Battery & [193] \\
\hline & Chitosan acetate-LiOAc-palmitic acid & Solid & $5.5 \times 10^{-6}$ & - & - & - & - & - & - & [194] \\
\hline & Chitosan- $\mathrm{LiCF}_{3} \mathrm{SO}_{3}-\mathrm{EC}$ & Solid & $5.5 \times 10^{-6}$ & - & - & $0.44 \mathrm{eV}$ & - & Amorph & - & [195] \\
\hline & Chitosan-LiOAc-oleic acid & Solid & $10^{-5}$ & - & - & - & - & Amorph & - & [16] \\
\hline & Chitosan-LiOAc-EC & Solid & $7.6 \times 10^{-6}$ & - & - & - & - & - & - & [196] \\
\hline & Chitosan acetate-LiN $\left(\mathrm{CF}_{3} \mathrm{SO}_{2}\right)_{2}$-oleic acid & Solid & $3.4 \times 10^{-6}$ & - & - & - & - & Amorph & - & [197] \\
\hline & Chitosan-KOH & Solid & $10^{-2}$ & - & - & - & - & - & Fuel cell & [198] \\
\hline & Chitosan acetate- $\mathrm{NH}_{4} \mathrm{NO}_{3}$ & Solid & $2.5 \times 10^{-5}$ & - & - & $0.45 \mathrm{eV}$ & - & Amorph & - & [17] \\
\hline & Chitosan acetate- $-\mathrm{NH}_{4} \mathrm{NO}_{3}-\mathrm{Al}_{2} \mathrm{SiO}_{3}$ & Solid & $2.1 \times 10^{-5}$ & - & - & - & - & - & - & [199] \\
\hline & Hexanoyl chitosan- $\mathrm{LiCF}_{3} \mathrm{SO}_{3}-\mathrm{EC} / \mathrm{PC}$ & Solid & $1.1 \times 10^{-4}$ & - & - & - & - & - & Battery & {$[200,201]$} \\
\hline & Chitosan acetate- $-\mathrm{NH}_{4} \mathrm{CF}_{3} \mathrm{SO}_{3}-\mathrm{DMC}$ & Solid & $10^{-6}$ & - & - & $0.60 \mathrm{eV}$ & - & - & - & [202] \\
\hline & Chitosan-LiOAc-oleic acid & & $1.1 \times 10^{-5}$ & - & - & $0.29 \mathrm{eV}$ & - & - & _ & \\
\hline & Chitosan-LiOAc-palmitic acid & Solid & $5.5 \times 10^{-6}$ & - & - & $0.45 \mathrm{eV}$ & - & - & - & [203] \\
\hline & Chitosan acetate- $-\mathrm{LiCF}_{3} \mathrm{SO}_{3}$ & & - & - & - & $0.38 \mathrm{eV}$ & - & - & Super-capacitor & [204] \\
\hline & Chitosan acetate- $\mathrm{H}_{3} \mathrm{PO}_{4}$ & Solid & & - & - & $0.49 \mathrm{eV}$ & - & - & Super-capacitor & [204] \\
\hline & Chitosan/glutaraldehyde- $\mathrm{KOH}$ & Solid & $10^{-2}$ & - & - & - & - & - & Fuel cell & [205] \\
\hline & Chitosan acetate- $\mathrm{NH}_{4} \mathrm{NO}_{3}-\mathrm{EC}$ & Solid & $9.9 \times 10^{-3}$ & - & - & - & - & - & Battery & [206] \\
\hline & Hexanoyl chitosan- $\mathrm{LiCF}_{3} \mathrm{SO}_{3}-\mathrm{EC}$ & Gel & $2.8 \times 10^{-5}$ & - & - & - & - & - & - & [207] \\
\hline & Chitosan/PEO-LiTFSI & Solid & $1.4 \times 10^{-6}$ & - & - & $0.64 \mathrm{eV}$ & - & - & - & [208] \\
\hline & Chitosan acetate- $-\mathrm{NH}_{4} \mathrm{NO}_{3}-\mathrm{O}-\mathrm{H}_{3} \mathrm{PO}_{4}$ & Solid & - & - & - & - & - & - & - & [209] \\
\hline & Chitosan/PEO-NH $\mathrm{NH}_{4} \mathrm{I} \mathrm{I}_{2}$ & Solid & $4.3 \times 10^{-6}$ & - & - & - & - & - & DSSC & [210] \\
\hline & Chitosan/PEO/pAPS-LiClO 4 & Solid & $1.7 \times 10^{-5}$ & - & - & - & - & - & - & [211] \\
\hline & Chitosan-HCl-glycerol & Solid & $2.2 \times 10^{-5}$ & - & - & $16.6 \mathrm{~kJ} / \mathrm{mol}$ & -87 & Amorph & - & [212] \\
\hline & Chitosan-PVPA & Solid & - & - & - & $0.32 \mathrm{eV}$ & - & Amorph & - & [213] \\
\hline & Chitosan acetate- $\mathrm{NH}_{4} \mathrm{NO}_{3}-\mathrm{EC}$ & Solid & $10^{-5}$ & - & 1.8 & $0.10 \mathrm{eV}$ & - & - & - & [214] \\
\hline & Chitosan acetate-apidic acid & Solid & $1.4 \times 10^{-9}$ & - & - & $0.52 \mathrm{eV}$ & - & - & - & [215] \\
\hline & Chitosan acetate- $\mathrm{NH}_{4} \mathrm{NO}_{3}-\mathrm{H}_{3} \mathrm{PO}_{4}-\mathrm{Al}_{2} \mathrm{SiO}_{3}$ & Solid & $1.8 \times 10^{-4}$ & - & - & - & - & Amorph & Fuel cell & [216] \\
\hline & Chitosan acetate/PEO- $\mathrm{NH}_{4} \mathrm{NO}_{3}-\mathrm{ES}$ & Solid & $10^{-4}$ & - & - & $0.02 \mathrm{eV}$ & - & - & - & [217] \\
\hline & Chitosan acetate- $\mathrm{CH}_{3} \mathrm{COONH}_{4}$ & Solid & $2.9 \times 10^{-4}$ & - & - & $0.19 \mathrm{eV}$ & - & Amorph & - & [218] \\
\hline & Hexanoyl chitosan- $-\mathrm{LiCF}_{3} \mathrm{SO}_{3}-\mathrm{EC}-\mathrm{Al}_{2} \mathrm{O}_{3}$ & Solid & $1.0 \times 10^{-4}$ & - & - & - & - & - & - & [219] \\
\hline & Hexanoyl chitosan- $-\mathrm{LiClO}_{4}-\mathrm{TiO}_{2}$ & Solid & $3.1 \times 10^{-4}$ & - & - & - & - & Amorph & - & {$[220,221]$} \\
\hline & Hexanoyl chitosan- $\mathrm{LiCF}_{3} \mathrm{SO}_{3}-\mathrm{DEC} / \mathrm{EC}$ & Solid & $4.3 \times 10^{-5}$ & - & - & $0.11 \mathrm{eV}$ & - & Semi-cr & - & [222] \\
\hline & Chitosan acetate- $\mathrm{NH}_{4} \mathrm{I}$-EC & Solid & $7.6 \times 10^{-6}$ & - & - & $0.21 \mathrm{eV}$ & - & - & - & [18] \\
\hline & Chitosan- $-\mathrm{LiClO}_{4}-\mathrm{EC} / \mathrm{PC}$ & Gel & $5.5 \times 10^{-3}$ & - & - & - & - & - & Super-capacitor & [223] \\
\hline & Chitosan acetate- $\mathrm{NH}_{4} \mathrm{I}-[\mathrm{BmIm}][\mathrm{I}]$ & Solid & $3.4 \times 10^{-5}$ & - & - & - & - & - & DSSC & {$[224,225]$} \\
\hline & Chitosan acetate- $-\mathrm{NH}_{4} \mathrm{Cl}$ & Solid & $5.4 \times 10^{-3}$ & - & - & $0.1 \mathrm{eV}$ & - & Semi-cr & - & [226] \\
\hline & Chitosan acetate- $-\mathrm{AgCF}_{3} \mathrm{SO}_{3}$ & Solid & - & - & - & $1.16 \mathrm{eV}$ & - & - & - & [227] \\
\hline & Chitosan acetate-NaI- $\mathrm{I}_{2}-[\mathrm{EmIm}][\mathrm{SCN}]$ & Solid & $2.6 \times 10^{-4}$ & - & - & - & - & Amorph & DSSC & [228] \\
\hline & $\mathrm{PVA} /$ chitosan acetate- $-\mathrm{NH}_{4} \mathrm{NO}_{3}$ & Solid & $1.6 \times 10^{-3}$ & - & - & $0.14 \mathrm{eV}$ & - & Amorph & Battery & [229] \\
\hline & Chitosan-g-PMMA-LiCF $3 \mathrm{SO}_{3}$ & Solid & $4.1 \times 10^{-5}$ & - & - & - & 110 & - & - & [230] \\
\hline & Chitosan/PEO-NH $\mathrm{NH}_{4} \mathrm{I}-\mathrm{I}_{2}-[\mathrm{BmIm}][\mathrm{I}]$ & gel & $5.5 \times 10^{-4}$ & - & - & - & - & - & DSSC & [231] \\
\hline & Chitosan acetate- $\mathrm{NH}_{4} \mathrm{SCN}-\mathrm{Al}_{2} \mathrm{O}_{3}$ & Solid & $5.9 \times 10^{-4}$ & - & - & - & 190 & Semi-cr & - & [232] \\
\hline & Hexanoyl chitosan- $\mathrm{LiClO}_{4}-\mathrm{TiO}_{2}$ & Solid & - & - & - & - & - & - & - & [233] \\
\hline
\end{tabular}


Table 3. Cont

\begin{tabular}{|c|c|c|c|c|c|c|c|c|c|c|}
\hline \multirow{2}{*}{ Polymer } & \multirow{2}{*}{ Electrolyte System } & \multirow{2}{*}{ State } & \multicolumn{4}{|c|}{ Electrochemical Properties } & \multicolumn{2}{|c|}{ Physical Properties } & \multirow{2}{*}{ Device } & \multirow{2}{*}{ Ref. } \\
\hline & & & $\sigma(\mathrm{s} / \mathrm{cm})$ & I-TN & Stability (V) & $E_{a}$ & $\mathrm{~T}_{\mathrm{g}}\left({ }^{\circ} \mathrm{C}\right)$ & Structural & & \\
\hline & Chitosan acetate/PEO- $\mathrm{NH}_{4} \mathrm{NO}_{3}$ & Solid & $1.0 \times 10^{-4}$ & - & - & - & - & Semi-cr & - & [234] \\
\hline & PVA/Chitosan- $\mathrm{NH}_{4} \mathrm{NO}_{3}-\mathrm{EC}$ & Solid & $1.6 \times 10^{-3}$ & - & 1.7 & - & - & - & EDLC & [235] \\
\hline & Chitosan- $-\mathrm{LiCF}_{3} \mathrm{SO}_{3}-\mathrm{EC} / \mathrm{PC}-\mathrm{SiO}_{2}$ & Solid & $4.4 \times 10^{-5}$ & - & - & $0.26 \mathrm{eV}$ & - & - & - & [236] \\
\hline & Chitosan/PVA-NH ${ }_{4} \mathrm{I}$ & Solid & $1.8 \times 10^{-6}$ & - & - & $0.38 \mathrm{eV}$ & - & Amorph & - & [237] \\
\hline & Chitosan acetate-glycerol & Solid & $1.1 \times 10^{-5}$ & - & - & - & -70 & - & - & [238] \\
\hline & Phthaloyl chitosan- $\mathrm{NH}_{4} \mathrm{SCN}$ & Solid & $2.4 \times 10^{-5}$ & - & 2.1 & $0.08 \mathrm{eV}$ & - & Amorph & - & [239] \\
\hline & Chitosan-[CBIm][Cl]- $\mathrm{I}_{2}$ & Solid & $9.1 \times 10^{-3}$ & - & - & - & - & - & - & [240] \\
\hline & ${\mathrm{Hexanoyl} \mathrm{chitosan-} \mathrm{LiClO}_{4}}$ & Solid & $4.2 \times 10^{-7}$ & - & - & - & - & - & - & [241] \\
\hline & $\begin{array}{l}\text { Hexanoyl chitosan- } \mathrm{LiCF}_{3} \mathrm{SO}_{3} \\
\text { Chitosan/PEO- } \mathrm{NH}_{4} \mathrm{NO}_{3}\end{array}$ & Solid & $\begin{array}{c}4.1 \times 10^{-6} \\
-\end{array}$ & _ & _ & $0.29 \mathrm{eV}$ & _- & _- & $\ldots$ & {$[242,243]$} \\
\hline & Nano-chitosan/PEO-LiCF $\mathrm{SO}_{3}$ & Solid & $10^{-3}$ & - & - & - & - & Semi-cr & - & {$[244]$} \\
\hline & $\mathrm{PEO} /$ Chitosan- $-\mathrm{NH}_{4} \mathrm{I}-\mathrm{I}_{2}$ & Solid & $1.2 \times 10^{-5}$ & - & - & - & - & - & DSSC & [245] \\
\hline & $\mathrm{CMCh}-\mathrm{ClCH}_{2} \mathrm{COOH}$ & Solid & $2 \times 10^{-7}$ & - & - & - & - & - & - & {$[246,247]$} \\
\hline & Chitosan/PEO-LiClO ${ }_{4}-\mathrm{EC} / \mathrm{PC}$ & Solid & $1.1 \times 10^{-4}$ & - & - & $0.12 \mathrm{eV}$ & - & - & Super-capacitor & [248] \\
\hline & Hexanoyl chitosan- $-\mathrm{LiCF}_{3} \mathrm{SO}_{3}-\mathrm{EC}-\mathrm{Al}_{2} \mathrm{O}_{3}$ & Solid & - & - & - & - & - & - & - & [249] \\
\hline & Chitosan- $\mathrm{NH}_{4} \mathrm{Br}$-glycerol & Solid & $2.2 \times 10^{-4}$ & - & - & $0.20 \mathrm{eV}$ & - & Amorph & - & [250] \\
\hline & Chitosan- $\mathrm{NH}_{4} \mathrm{SCN}-\mathrm{Al}_{2} \mathrm{TiO}_{5}$ & Solid & $2.1 \times 10^{-4}$ & - & - & - & - & Amorph & - & [251] \\
\hline & Chitosan/PEO-NH $\mathrm{NO}_{3}-\mathrm{EC}$ & Solid & $2.1 \times 10^{-3}$ & - & 1.75 & $0.18 \mathrm{eV}$ & - & Amorph & EDLC & [64] \\
\hline & Methyl cellulose/chitosan- $\mathrm{NH}_{4} \mathrm{CF}_{3} \mathrm{SO}_{3}$ & Solid & $5.0 \times 10^{-6}$ & - & - & - & - & - & - & [252] \\
\hline & $\mathrm{CMCh}-\mathrm{NH}_{4} \mathrm{CF}_{3} \mathrm{SO}_{3}$ & Solid & $8.9 \times 10^{-6}$ & - & 0.8 & - & - & - & - & [253] \\
\hline & Chitosan-[EmIm][C $\left.\mathrm{C}_{1} \mathrm{SO}_{3}\right]$-glycerol & & $7.8 \times 10^{-4}$ & - & - & $12.1 \mathrm{~kJ} / \mathrm{mol}$ & - & - & & \\
\hline & Chitosan-[EmIm] $\left[\mathrm{C}_{2} \mathrm{SO}_{3}\right.$-glycerol & Solid & $4.2 \times 10^{-4}$ & - & - & $14.3 \mathrm{~kJ} / \mathrm{mol}$ & - & - & - & [254] \\
\hline & Chitosan-[EmIm] [C $\left.4 \mathrm{SO}_{3}\right]$-glycerol & & $1.5 \times 10^{-4}$ & - & - & $16.7 \mathrm{~kJ} / \mathrm{mol}$ & - & - & & \\
\hline & Hexanoyl chitosan- $\mathrm{LiClO}_{4}-\mathrm{TiO}_{2}$ & & $3.1 \times 10^{-4}$ & - & - & $0.08 \mathrm{eV}$ & - & - & _- & \\
\hline & Hexanoyl chitosan- $\mathrm{LiClO}_{4}-\mathrm{SiO}_{2}$ & Solid & $2.0 \times 10^{-4}$ & - & - & $0.12 \mathrm{eV}$ & - & - & - & [255] \\
\hline & Chitosan-g-PMMA- $\mathrm{LiCF}_{3} \mathrm{SO}_{3}-\mathrm{EC}$ & Solid & $2.2 \times 10^{-4}$ & - & - & - & - & - & - & [256] \\
\hline & Chitosan-LiTFSI-succinonitrile & Solid & $0.4 \times 10^{-3}$ & - & 4.7 & - & - & Amorph & Battery & [257] \\
\hline & CMC/chitosan- $-\mathrm{NH}_{4} \mathrm{Br}$ & Solid & $1.2 \times 10^{-5}$ & - & - & - & - & - & - & [258] \\
\hline & Hexanoyl chitosan/polystyrene- $-\mathrm{LiCF}_{3} \mathrm{SO}_{3}-\mathrm{TiO}_{2}$ & Solid & $2.8 \times 10^{-4}$ & - & - & - & - & Amorph & - & [259-261] \\
\hline & PVA/chitosan- $\mathrm{NH}_{4} \mathrm{Br}$ & Solid & $7.7 \times 10^{-4}$ & - & 1.6 & $0.15 \mathrm{eV}$ & - & Amorph & - & [262] \\
\hline & Corn starch/chitosan- $\mathrm{NH}_{4} \mathrm{I}$ & Solid & $3.0 \times 10^{-4}$ & - & - & $0.20 \mathrm{eV}$ & - & Amorph & - & [92] \\
\hline & Chitosan/gold- $-\mathrm{LiClO}_{4}$ & Solid & $7.2 \times 10^{-7}$ & - & - & - & - & Amorph & - & [263] \\
\hline & Phosphorylated chitosan- $\mathrm{LiClO}_{4}$ & Solid & $1.4 \times 10^{-3}$ & - & - & - & - & - & - & [264] \\
\hline & Chitosan-Oxalic acid & Solid & $5.0 \times 10^{-7}$ & - & - & $0.61 \mathrm{eV}$ & - & Amorph & - & {$[265,266]$} \\
\hline & N-Succinyl chitosan- $\mathrm{LiClO}_{4}$ & Solid & $8.0 \times 10^{-3}$ & - & - & - & - & - & - & {$[267,268]$} \\
\hline & Hexanoyl chitosan- $-\mathrm{LiClO}_{4}$-DMC & Solid & $10^{-4}$ & - & - & $0.06 \mathrm{eV}$ & - & - & - & [269] \\
\hline & Hexanoyl chitosan- $\mathrm{LiClO}_{4}-\mathrm{DMC}-\mathrm{TiO}_{2}$ & Solid & $4.1 \times 10^{-4}$ & - & - & - & - & Amorph & - & [270] \\
\hline & Lauroyl chitosan/PMMA-LiCF $\mathrm{PO}_{3}-\mathrm{EC}$ & Solid & $7.6 \times 10^{-4}$ & - & - & - & - & Amorph & - & [271] \\
\hline & NSB-Chitosan-NMPS-GO & Solid & $8.9 \times 10^{-2}$ & - & - & $4.57 \mathrm{~kJ} / \mathrm{mol}$ & - & - & - & [272] \\
\hline & Methyl cellulose/chitosan- $\mathrm{NH}_{4} \mathrm{CF}_{3} \mathrm{SO}_{3}-[\mathrm{BmIm}][\mathrm{TFSI}]$ & Solid & $4.0 \times 10^{-4}$ & - & - & - & - & - & - & [273] \\
\hline & Starch/chitosan-NH ${ }_{4} \mathrm{Cl}$-glycerol & Solid & $5.1 \times 10^{-4}$ & 0.97 & 1.65 & - & - & Amorph & Battery & [274] \\
\hline & Chitosan acetate-LiCl & Gel & $2.9 \times 10^{-3}$ & - & - & $0.20 \mathrm{eV}$ & - & - & - & [275] \\
\hline & Chitosan-[BmIm][OAc] & Solid & $2.4 \times 10^{-3}$ & 0.75 & 3.4 & $0.29 \mathrm{eV}$ & 35 & Amorph & - & [276] \\
\hline & Hexanoyl chitosan- $\mathrm{LiClO}_{4} / \mathrm{TiO}_{2}$ & Solid & $3.0 \times 10^{-4}$ & - & - & - & - & - & - & [277] \\
\hline & PVA/chitosan-[BmIm][Br] & & $4.2 \times 10^{-2}$ & 0.65 & - & - & - & - & _ & \\
\hline & $\mathrm{PVA} /$ chitosan-[EmIm][Cl] & Solid & $5.5 \times 10^{-2}$ & 0.70 & - & - & - & - & - & [278] \\
\hline
\end{tabular}


Table 3. Cont

\begin{tabular}{|c|c|c|c|c|c|c|c|c|c|c|}
\hline \multirow{2}{*}{ Polymer } & \multirow{2}{*}{ Electrolyte System } & \multirow{2}{*}{ State } & \multicolumn{4}{|c|}{ Electrochemical Properties } & \multicolumn{2}{|c|}{ Physical Properties } & \multirow{2}{*}{ Device } & \multirow{2}{*}{ Ref. } \\
\hline & & & $\sigma(\mathrm{s} / \mathrm{cm})$ & I-TN & Stability (V) & $\mathrm{E}_{\mathrm{a}}$ & $\mathrm{T}_{\mathrm{g}}\left({ }^{\circ} \mathrm{C}\right)$ & Structural & & \\
\hline & Chitosan- $\mathrm{NaCF}_{3} \mathrm{SO}_{3}-\mathrm{Al}_{2} \mathrm{O}_{3}$ & Solid & - & - & - & - & - & Amorph & - & [279] \\
\hline & 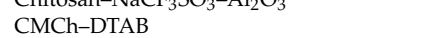 & Solid & $1.9 \times 10^{-6}$ & - & - & - & - & - & - & [280] \\
\hline & Chitosan/PEO-NH 4 I & Solid & $3.7 \times 10^{-6}$ & 0.85 & - & - & - & Amorph & DSSC & [281] \\
\hline & Chitosan- $\mathrm{LiClO}_{4}-\mathrm{ZrO}_{2}$ & Solid & $3.6 \times 10^{-4}$ & 0.55 & - & - & - & Amorph & - & [282] \\
\hline & Chitosan-perchloric acid & Solid & $5.9 \times 10^{-4}$ & - & - & - & - & - & - & [283] \\
\hline & $\mathrm{N}-\mathrm{phth}$ loyl chitosan-TPAI- $\mathrm{I}_{2}-\mathrm{EC}$ & Solid & $5.5 \times 10^{-3}$ & - & - & $0.11 \mathrm{eV}$ & - & Amorph & DSSC & [284] \\
\hline & Chitosan-oxalic acid & Solid & $4.1 \times 10^{-5}$ & - & - & - & - & - & - & [285] \\
\hline & Sulfonated chitosan-sulfonated GO & Solid & $7.2 \times 10^{-3}$ & - & - & - & - & - & - & [286] \\
\hline & Chitosan- $\mathrm{LiCF}_{3} \mathrm{SO}_{3}-\mathrm{Al}_{2} \mathrm{O}_{3}$ & Solid & $10^{-6}$ & - & - & - & - & Amorph & - & [287] \\
\hline & Chitosan-Ce $\left(\mathrm{CF}_{3} \mathrm{SO}_{3}\right)_{3}$-glycerol & Solid & $1.7 \times 10^{-5}$ & - & - & - & - & Amorph & - & [288] \\
\hline & Chitosan-Eu $\left(\mathrm{CF}_{3} \mathrm{SO}_{3}\right)_{3}$-glycerol & Solid & $1.5 \times 10^{-6}$ & - & - & - & - & Amorph & - & [289] \\
\hline & Chitosan- $\mathrm{NaCF}_{3} \mathrm{SO}_{3}$ & Solid & $2.4 \times 10^{-4}$ & - & - & $0.3 \mathrm{eV}$ & - & Amorph & - & [290] \\
\hline & Chitosan/pectin- $\mathrm{HCl}$ & Solid & $2.4 \times 10^{-3}$ & - & - & - & - & - & - & [291] \\
\hline & Chitosan- $\mathrm{Mg}\left(\mathrm{CF}_{3} \mathrm{SO}_{3}\right)_{2}-[\mathrm{EmIm}]\left[\mathrm{CF}_{3} \mathrm{SO}_{3}\right]$ & Solid & $3.6 \times 10^{-5}$ & 0.98 & 4.15 & $0.72 \mathrm{eV}$ & - & - & - & [292] \\
\hline & Hexanoyl chitosan-NaI & & $1.3 \times 10^{-6}$ & - & - & - & -24 & Amorph & _ & [2931 \\
\hline & Lauroyl chitosan-NaI & Solid & $1.1 \times 10^{-8}$ & - & - & - & -10 & Amorph & - & [293] \\
\hline & Chitosan- $\mathrm{AgCF}_{3} \mathrm{SO}_{3}-\mathrm{Al}_{2} \mathrm{O}_{3}$ & Solid & - & - & - & - & - & - & - & [294] \\
\hline & Chitosan- $\mathrm{Tm}\left(\mathrm{CF}_{3} \mathrm{SO}_{3}\right)_{3}$-glycerol & Solid & $10^{-5}$ & - & - & - & - & Amorph & $\mathrm{ECD}$ & [295] \\
\hline & Chitosan-[EmIm] [Eu(SCN $\left.)_{4}\right]$ & Solid & $1.3 \times 10^{-5}$ & - & - & - & - & Semi-cr & - & [296] \\
\hline & $\begin{array}{l}\text { Chitosan-[EmIm][SCN] } \\
\text { (ETI }\end{array}$ & Solid & $1.6 \times 10^{-3}$ & - & 4.0 & - & - & Amorph & - & {$[297,298]$} \\
\hline \multirow{27}{*}{ Agar } & Agar-Acetic acid & Solid & $1.1 \times 10^{-4}$ & - & - & $33.5 \mathrm{~kJ} / \mathrm{mol}$ & - & Amorph & - & [11] \\
\hline & Agar-LiI- $\mathrm{I}_{2}-\mathrm{TiO}_{2}$ & Gel & $5.1 \times 10^{-4}$ & - & - & - & - & - & DSSC & [299] \\
\hline & Agar-Lil- $\mathrm{I}_{2}-\mathrm{TiO}_{2}$ & Gel & $4.0 \times 10^{-4}$ & - & - & - & - & - & DSSC & [300] \\
\hline & Agar-Eu(pic) ${ }_{3}$-glycerol & Solid & $1.6 \times 10^{-5}$ & - & - & - & - & Amorph & $\mathrm{ECD}$ & [301] \\
\hline & Agar-LiClO 4 -glycerol & Gel & $6.5 \times 10^{-5}$ & - & - & - & - & Amorph & ECD & [26] \\
\hline & Agar-[EmIm] [C $\left.{ }_{2} \mathrm{SO}_{4}\right]$-glycerol & & - & - & - & - & - & Amorph & & \\
\hline & Agar-[EmIm][OAc]-glycerol & Solid & $2.4 \times 10^{-5}$ & - & - & $24.3 \mathrm{~kJ} / \mathrm{mol}$ & - & Amorph & ECD & [302] \\
\hline & Agar-[Ch][OAc]-glycerol & & - & - & - & - & - & Amorph & & \\
\hline & Agar-LiI- $\mathrm{I}_{2}-\mathrm{NiO}$ & Gel & - & - & - & - & - & - & DSSC & [303] \\
\hline & Agar-LiI- $\mathrm{I}_{2}-\mathrm{Fe}_{3} \mathrm{O}_{4}-\mathrm{PEG}$ & Gel & $2.9 \times 10^{-3}$ & - & - & - & - & - & DSSC & [304] \\
\hline & Agar-LiI- $\mathrm{I}_{2}-\mathrm{Fe}_{3} \mathrm{O}_{4}-\mathrm{SDS}$ & & - & - & - & - & - & - & & \\
\hline & Agar-LiI- $\mathrm{I}_{2}-\mathrm{Fe}_{3} \mathrm{O}_{4}-\mathrm{PVP}$ & Gel & - & - & - & - & - & - & DSSC & [305] \\
\hline & Agar-LiI- $\mathrm{I}_{2}-\mathrm{Fe}_{3} \mathrm{O}_{4}-\mathrm{TW}-80$ & & $3.0 \times 10^{-3}$ & - & - & - & - & - & & \\
\hline & Bacto agar-NaI- $\mathrm{I}_{2}$ & Gel & $1.2 \times 10^{-3}$ & - & 2.0 & - & - & Amorph & - & [306] \\
\hline & Agar- $\mathrm{Mg}\left(\mathrm{CF}_{3} \mathrm{SO}_{3}\right)_{2}$-glycerol & Solid & $1.0 \times 10^{-6}$ & - & - & - & - & Amorph & ECD & [307] \\
\hline & Agar-LiI- $\mathrm{I}_{2}-\mathrm{TiO}_{2}$ & & $2.7 \times 10^{-3}$ & - & - & - & - & - & & \\
\hline & Agar-LiI- $\mathrm{I}_{2}-\mathrm{Co}_{3} \mathrm{O}_{4}$ & Gel & $4.4 \times 10^{-3}$ & - & - & - & - & - & DSSC & [308] \\
\hline & Agar-LiI- $\mathrm{I}_{2}-\mathrm{NiO}$ & & $3.3 \times 10^{-3}$ & - & - & - & - & - & & \\
\hline & Agar-NiO-glycerol-acetic acid & Solid & $5.2 \times 10^{-5}$ & - & - & - & - & Amorph & - & [65] \\
\hline & Agar-LiClO 4 -glycerol & & $6.5 \times 10^{-8}$ & _- & _- & $0.1 \mathrm{eV}$ & _- & Amorph & & \\
\hline & Agar- $\mathrm{KClO}_{4}$-glycerol & Solid & $9.1 \times 10^{-8}$ & - & - & $0.1 \mathrm{eV}-$ & _- & Amorph & - & ] \\
\hline & Agar-Acetic acid-glycerol & Solid & $3.5 \times 10^{-8}$ & - & - & $0.1 \mathrm{eV}$ & - & Amorph & - & [25] \\
\hline & Agar-Lactic acid-glycerol & & $2.2 \times 10^{-8}$ & - & - & $0.1 \mathrm{eV}$ & - & Amorph & & \\
\hline & Agar- $\mathrm{NH}_{4} \mathrm{NO}_{3}$ & Solid & $6.6 \times 10^{-4}$ & 0.99 & - & $0.12 \mathrm{eV}$ & - & Amorph & Fuel cell & [309] \\
\hline & Agar- $-\mathrm{Na}_{2} \mathrm{~S} / \mathrm{S}$-glycerol & Gel & $1.8 \times 10^{-3}$ & - & - & - & - & - & DSSC & [310] \\
\hline & Agar- $\mathrm{NH}_{4} \mathrm{SCN}$ & Solid & $1.0 \times 10^{-3}$ & 0.97 & - & $0.25 \mathrm{eV}$ & 55 & Amorph & - & [311] \\
\hline & Agar- $\mathrm{NH}_{4} \mathrm{I}$ & Solid & $1.1 \times 10^{-4}$ & - & - & $0.43 \mathrm{eV}$ & - & Amorph & - & [312] \\
\hline
\end{tabular}


Table 3. Cont

\begin{tabular}{|c|c|c|c|c|c|c|c|c|c|c|}
\hline \multirow{2}{*}{ Polymer } & \multirow{2}{*}{ Electrolyte System } & \multirow{2}{*}{ State } & \multicolumn{4}{|c|}{ Electrochemical Properties } & \multicolumn{2}{|c|}{ Physical Properties } & \multirow{2}{*}{ Device } & \multirow[b]{2}{*}{ Ref. } \\
\hline & & & $\sigma(\mathrm{s} / \mathrm{cm})$ & I-TN & Stability (V) & $E_{a}$ & $\mathrm{~T}_{\mathrm{g}}\left({ }^{\circ} \mathrm{C}\right)$ & Structural & & \\
\hline & Agar-KI-MPII & Gel & $1.5 \times 10^{-3}$ & - & - & - & - & Amorph & DSSC & [313] \\
\hline \multirow{14}{*}{ Carrageenan } & Carboxymethyl k-carrageenan-acetic acid & Solid & $2.0 \times 10^{-4}$ & - & - & - & - & - & - & [29] \\
\hline & $k$-carrageenan-[Bmim]Cl & Solid & $2.4 \times 10^{-3}$ & - & - & - & - & Amorph & - & [314] \\
\hline & Carboxymethyl t-carrageenan-acetic acid & Solid & $4.9 \times 10^{-6}$ & - & - & - & - & Amorph & - & [315] \\
\hline & Carboxymethyl k-carrageenan/CMC- $\mathrm{NH}_{4} \mathrm{I}$ & Solid & $2.4 \times 10^{-3}$ & 0.99 & 2.0 & $0.01 \mathrm{eV}$ & - & - & DSSC & [316] \\
\hline & k-carrageenan- $\mathrm{TBAI}-\mathrm{I}_{2}-\mathrm{TiO}_{2}$ & & & - & 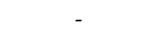 & - & - & - & & \\
\hline & k-carrageenan- $-\mathrm{TBAI}-\mathrm{I}_{2}-\mathrm{Fe}_{2} \mathrm{O}_{3}$ & Solid & - & - & - & - & - & - & DSSC & [317] \\
\hline & k-carrageenan-TBAI- $\mathrm{I}_{2}$-halloysite clay & & & - & - & - & - & - & & \\
\hline & Carboxymethyl k-carrageenan/CMC-acetic acid & Solid & $3.3 \times 10^{-4}$ & - & 2.75 & - & -13.5 & - & - & [30] \\
\hline & Carboxymethyl k-carrageenan-LiNO 3 & & $5.9 \times 10^{-3}$ & - & 3.1 & $0.18 \mathrm{eV}$ & - & - & _ & \\
\hline & Carboxymethyl l-carrageenan-LiNO 3 & Solid & $5.5 \times 10^{-3}$ & - & 3.0 & $0.38 \mathrm{eV}$ & - & - & - & [22] \\
\hline & Carboxymethyl k-carrageenan/CMC-LiI- $\mathrm{I}_{2}$ & Solid & $3.9 \times 10^{-3}$ & - & - & $0.01 \mathrm{eV}$ & -43.0 & - & DSSC & [318] \\
\hline & t-carrageenan- $\mathrm{NH}_{4} \mathrm{Br}$ & Solid & $1.1 \times 10^{-3}$ & - & 2.1 & $0.18 \mathrm{eV}$ & - & Amorph & Fuel cell & [319] \\
\hline & $\mathrm{k}$-carrageenan/PEDOT-PANI & Gel & - & - & - & - & - & - & Super-capacitor & [320] \\
\hline & t-carrageenan- $\mathrm{NH}_{4} \mathrm{NO}_{3}$ & Solid & $1.5 \times 10^{-3}$ & 0.95 & 2.46 & $0.14 \mathrm{eV}$ & 64 & - & ECD & [321] \\
\hline \multirow{7}{*}{ Pectin } & Pectin-LiClO 4 & Solid & $4.7 \times 10^{-4}$ & - & - & - & - & Amorph & - & [33] \\
\hline & Pectin-HCl-glutaraldehyde & Solid & $2.5 \times 10^{-2}$ & - & - & - & - & Amorph & - & [322] \\
\hline & Pectin-KCl-glycerol & & $1.5 \times 10^{-3}$ & - & - & - & - & Amorph & _- & \\
\hline & Pectin-KCl-Ir(III)-glycerol & Solid & $5.4 \times 10^{-5}$ & - & - & - & - & Amorph & - & [323] \\
\hline & Pectin- $\mathrm{NH}_{4} \mathrm{Cl}$ & & $4.5 \times 10^{-4}$ & - & - & - & - & Amorph & 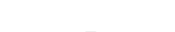 & 1324 \\
\hline & Pectin- $\mathrm{NH}_{4} \mathrm{Br}$ & Solid & $1.1 \times 10^{-3}$ & - & - & - & - & Amorph & - & [324] \\
\hline & Pectin-[ $\left.\mathrm{N}_{1112(\mathrm{OH})}\right]\left[\mathrm{NTf}_{2}\right]$-glycerol & Solid & $1.4 \times 10^{-6}$ & - & - & - & - & Amorph & - & [325] \\
\hline \multirow{4}{*}{ Guar gum and gum arabic } & Guar gum--LiClO ${ }_{4}$-glycerol & Solid & $2.2 \times 10^{-3}$ & - & - & $0.18 \mathrm{eV}$ & - & - & - & [37] \\
\hline & Guar gum-[BmIm][Cl]-PEDOT & Gel & $10^{-2}$ & - & - & - & - & - & - & [35] \\
\hline & Guar gum-[BmIm][Cl]-P(AEMIBr) & Gel & $10^{-4}$ & - & - & - & - & - & - & [38] \\
\hline & Gum Arabic-o- $\mathrm{H}_{3} \mathrm{PO}_{4}$ & Gel & $1.8 \times 10^{-2}$ & - & - & - & - & - & Super-capacitor & [326] \\
\hline \multirow{19}{*}{ Gelatin } & Gelatin-glycerol-formaldehyde-acetic acid & Solid & $4.5 \times 10^{-5}$ & - & - & $32.6 \mathrm{~kJ} / \mathrm{mol}$ & - & - & - & [42] \\
\hline & Gelatin- $\mathrm{LiClO}_{4}$-glycerol & Solid & $10^{-4}$ & - & - & $0.35 \mathrm{eV}$ & - & - & - & [327] \\
\hline & Gelatin-LiBF 4 -glycerol & Gel & $2.3 \times 10^{-5}$ & - & - & - & - & - & & \\
\hline & Gelatin-LiClO 4 -glycerol & Gel & $3.2 \times 10^{-5}$ & - & - & - & - & - & _- & \\
\hline & Gelatin-HCl-glycerol & Gel & $5.4 \times 10^{-5}$ & - & - & - & - & - & - & [41] \\
\hline & Gelatin-Acetic acid-glycerol & Gel & $8.7 \times 10^{-4}$ & - & - & _- & _- & _- & & \\
\hline & Gelatin-LiClO 4 -EC/PC & Solid & $2.0 \times 10^{-9}$ & - & - & - & - & - & - & [328] \\
\hline & Gelatin-LiClO 4 & Solid & - & - & - & - & - & - & ECD & [329] \\
\hline & Poly(acrylic acid-g-gelatin)/polypyrrole-KI- $\mathrm{I}_{2}$ & Gel & $1.4 \times 10^{-2}$ & - & - & $10.3 \mathrm{~kJ} / \mathrm{mol}$ & - & - & DSSC & [330] \\
\hline & $\begin{array}{l}\text { Gelatin-Acetic acid-glycerol }\end{array}$ & Solid & $2 \times 10^{-5}$ & - & - & $0.22 \mathrm{eV}$ & - & - & - & [331] \\
\hline & Gelatin-LiBF - -glycerol & Gel & $1.5 \times 10^{-5}$ & - & - & $43.1 \mathrm{~kJ} / \mathrm{mol}$ & - & Amorph & - & [332] \\
\hline & Gelatin-[Eu(pic) $\left.)_{3}\right]$-glycerol & Solid & - & - & - & - & - & Amorph & ECD & [333] \\
\hline & Gelatin-HCl-glycerol & Solid & $4.0 \times 10^{-5}$ & - & - & $23 \mathrm{~kJ} / \mathrm{mol}$ & - & - & - & [334] \\
\hline & Gelatin-[EmIm][OAc] & Solid & $1.2 \times 10^{-4}$ & - & - & $16.7 \mathrm{~kJ} / \mathrm{mol}$ & - & Amorph & $\mathrm{ECD}$ & [335] \\
\hline & Gelatin-LiI-I $I_{2}$ & Solid & $5 \times 10^{-5}$ & - & - & $8 \mathrm{~kJ} / \mathrm{mol}$ & -76 & Amorph & ECD & [336] \\
\hline & Gelatin- $\mathrm{LiClO}_{4}$-glycerol & Solid & $10^{-4}$ & - & - & - & - & - & - & {$[337,338]$} \\
\hline & Gelatin-Glycerol & Solid & $9.1 \times 10^{-3}$ & - & - & - & - & Amorph & - & [339] \\
\hline & Gelatin- $\mathrm{Zn}\left(\mathrm{CF}_{3} \mathrm{SO}_{3}\right)_{2}$ & Solid & $3.1 \times 10^{-10}$ & - & - & - & - & Amorph & - & [340] \\
\hline & Gelatin-LiClO ${ }_{4}$-glycerol & - & $1.1 \times 10^{-4}$ & - & - & $9.37 \mathrm{~kJ} / \mathrm{mol}$ & - & Amorph & _- & {$[341]$} \\
\hline
\end{tabular}


Table 3. Cont

\begin{tabular}{|c|c|c|c|c|c|c|c|c|c|c|}
\hline \multirow{2}{*}{ Polymer } & \multirow{2}{*}{ Electrolyte System } & \multirow{2}{*}{ State } & \multicolumn{4}{|c|}{ Electrochemical Properties } & \multicolumn{2}{|c|}{ Physical Properties } & \multirow{2}{*}{ Device } & \multirow{2}{*}{ Ref. } \\
\hline & & & $\sigma(\mathrm{s} / \mathrm{cm})$ & I-TN & Stability (V) & $E_{a}$ & $\mathrm{~T}_{\mathrm{g}}\left({ }^{\circ} \mathrm{C}\right)$ & Structural & & \\
\hline & Gelatin-LiCl-glycerol & & $2.0 \times 10^{-4}$ & - & - & $6.31 \mathrm{~kJ} / \mathrm{mol}$ & - & Amorph & & \\
\hline & Gelatin- $\mathrm{Mg}\left(\mathrm{CF}_{3} \mathrm{SO}_{3}\right)_{2}$-glycerol & Solid & $3.8 \times 10^{-10}$ & - & - & $49.0 \mathrm{~kJ} / \mathrm{mol}$ & - & Amorph & - & [39] \\
\hline & Gelatin/Au-LiI- $\mathrm{I}_{2} / 2$. & Gel & $2.2 \times 10^{-2}$ & - & - & - & 77 & - & DSSC & [342] \\
\hline & Gelatin-[EmIm][N(CN) $\left.)_{2}\right]$-glycerol & Solid & $2.4 \times 10^{-3}$ & - & - & - & - & Amorph & $\mathrm{ECD}$ & [343] \\
\hline & Gelatin-NaCl & Gel & $8.5 \times 10^{-2}$ & - & - & - & - & - & - & [110] \\
\hline \multirow{34}{*}{ Natural rubber } & NR/PEO-LiBs-PEO 1000 & Solid & $10^{-6}$ & - & - & - & - & - & - & [44] \\
\hline & ENR-25- $-\mathrm{LiCF}_{3} \mathrm{SO}_{3}-\mathrm{EC} / \mathrm{PC}$ & & $2.9 \times 10^{-4}$ & - & - & - & -43 & - & & \\
\hline & ENR-50- $\mathrm{LiCF}_{3} \mathrm{SO}_{3}-\mathrm{EC} / \mathrm{PC}$ & Solid & $1.3 \times 10^{-4}$ & - & - & - & -35 & - & - & [344] \\
\hline & MG-49-- $\mathrm{LiCF}_{3} \mathrm{SO}_{3}-\mathrm{EC} / \mathrm{PC}$ & & $4.3 \times 10^{-4}$ & - & - & - & - & - & & \\
\hline & ENR-25/PEO-LiCF ${ }_{3} \mathrm{SO}_{3}-\mathrm{EC} / \mathrm{PC}$ & & $10^{-4}$ & - & - & - & - & - & 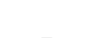 & \\
\hline & ENR-50/PEO-LiCF ${ }_{3} \mathrm{SO}_{3}-\mathrm{EC} / \mathrm{PC}$ & Solid & $10^{-4}$ & - & - & - & - & - & - & [345] \\
\hline & MG-30- $\mathrm{LiCF}_{3} \mathrm{SO}_{3}-\mathrm{EC}-\mathrm{Al}_{2} \mathrm{SiO}_{5}$ & Solid & - & - & - & - & -41 & - & - & [346] \\
\hline & PVC/ENR-50-LiCF ${ }_{3} \mathrm{SO}_{3}$ & & $3.6 \times 10^{-5}$ & - & - & - & - & - & _- & \\
\hline & PMMA/ENR-50--LiCF $\mathrm{SO}_{3}$ & Solid & $5.1 \times 10^{-5}$ & - & - & - & - & - & - & [347] \\
\hline & MG-30- $\mathrm{LiCF}_{3} \mathrm{SO}_{3}$ & & $8.4 \times 10^{-4}$ & - & 4.3 & - & - & - & & \\
\hline & $\mathrm{MG}-30-\mathrm{LiCF}_{3} \mathrm{SO}_{3}$-EC & Gel & $0.4 \times 10$ & - & - & - & - & - & - & [348] \\
\hline & $\mathrm{MG}-30-\mathrm{LiCF}_{3} \mathrm{SO}_{3}-\mathrm{PC}$ & & - & - & - & - & - & - & & \\
\hline & PMMA/ENR-50- $\mathrm{LiCF}_{3} \mathrm{SO}_{3}-\mathrm{EC}$ & Solid & - & - & - & - & - & - & - & [349] \\
\hline & MG-49- $\mathrm{NH}_{4} \mathrm{CF}_{3} \mathrm{SO}_{3}-\mathrm{PC}$ & Gel & $3.3 \times 10^{-2}$ & - & - & - & - & - & Battery & [350] \\
\hline & MG-49-LiBF 4 & Solid & $2.3 \times 10^{-7}$ & - & - & - & - & Amorph & - & [251 \\
\hline & MG-49-- $\mathrm{LiClO}_{4}$ & Solid & $4.0 \times 10^{-8}$ & - & - & - & - & Amorph & & [351] \\
\hline & MG-49-- $\mathrm{LiClO}_{4}-\mathrm{EC}-\mathrm{TiO}_{2}$ & Solid & $1.1 \times 10^{-3}$ & - & - & - & - & - & - & [352] \\
\hline & MG-49- $\mathrm{NH}_{4} \mathrm{CF}_{3} \mathrm{SO}_{3}-\mathrm{SiO}_{3}$ & Gel & $7.6 \times 10^{-3}$ & - & - & - & - & - & - & [353] \\
\hline & PMMA/MG-49-LiBF 4 & Solid & $8.3 \times 10^{-6}$ & - & - & - & - & Amorph & - & [354] \\
\hline & MG-49- $\mathrm{NH}_{4} \mathrm{CF}_{3} \mathrm{SO}_{3}-\mathrm{PC}$ & Gel & $2.9 \times 10^{-2}$ & - & - & - & - & - & - & [355] \\
\hline & MG-30- $\mathrm{NH}_{4} \mathrm{CF}_{3} \mathrm{SO}_{3}-\mathrm{EC}$ & Solid & $10^{-4}$ & - & - & - & - & Amorph & - & [356] \\
\hline & PVC/LENR-50-LiClO & Solid & $2.3 \times 10^{-8}$ & - & - & - & - & Amorph & - & [357] \\
\hline & ENR-50- $\mathrm{Li}_{2} \mathrm{NH}$ & Solid & $3.5 \times 10^{-5}$ & - & - & - & - & - & - & [358] \\
\hline & MG-49/Cellulose--LiCF $\mathrm{SO}_{3}$ & Solid & $5.3 \times 10^{-7}$ & - & - & - & - & Amorph & - & [359] \\
\hline & $\mathrm{MG}-30-\mathrm{LiCF}_{3} \mathrm{SO}_{3}-\mathrm{EC}$ & Gel & $9.0 \times 10^{-3}$ & - & 4.2 & $0.14 \mathrm{eV}$ & -77.2 & - & Battery & [360] \\
\hline & PVDF-HFP/MG-49-LiCF $\mathrm{SO}_{3}$ & Solid & $2.0 \times 10^{-4}$ & - & 3.0 & $0.14 \mathrm{eV}$ & - & Semi-cr & Daty & [361-363] \\
\hline & ENR-50-LiN $\left(\mathrm{SO}_{2} \mathrm{CF}_{3}\right)_{2}-\mathrm{EC} / \mathrm{PC}$ & Solid & $2.6 \times 10^{-4}$ & - & - & - & -46.8 & - & - & [364] \\
\hline & PVDF/MG-49- $-\mathrm{NH}_{4} \mathrm{CF}_{3} \mathrm{SO}_{3}$ & Solid & $6.3 \times 10^{-4}$ & - & 4.0 & - & - & Semi-cr & - & [365] \\
\hline & $\mathrm{MG}-49 / \mathrm{CMC}-\mathrm{LiCF}_{3} \mathrm{SO}_{3}$ & Solid & $3.3 \times 10^{-7}$ & - & 2.7 & - & - & - & - & [366] \\
\hline & $\mathrm{MG}-49-\mathrm{LiCF}_{3} \mathrm{SO}_{3}-\mathrm{ZrO}_{2} / \mathrm{TiO}_{2}$ & Solid & $1.2 \times 10^{-5}$ & - & - & $0.10 \mathrm{eV}$ & - & Amorph & Battery & [367] \\
\hline & $\mathrm{MG}-30-\mathrm{LiCF}_{3} \mathrm{SO}_{3}$ & Solid & $5.6 \times 10^{-3}$ & - & - & - & -43.9 & - & Daty & [368] \\
\hline & ENR-50--LiClO & Solid & $10^{-5}$ & - & - & - & -22 & - & - & [369] \\
\hline & ENR-25/hexanoyl chitosan-LiN $\left(\mathrm{CF}_{3} \mathrm{SO}_{2}\right)_{2}-[\mathrm{EmIm}][\mathrm{TFSI}]$ & Solid & $1.3 \times 10^{-6}$ & - & - & - & -31 & - & - & [370] \\
\hline & ENR-25- $-\mathrm{LiClO}_{4}$ & Solid & - & - & - & - & -42 & - & - & [371] \\
\hline \multirow{5}{*}{ Poly(lactic acid) } & PLA- $\mathrm{LiClO}_{4}-\mathrm{EC}-\mathrm{Al}_{2} \mathrm{O}_{3}$ & & $2.1 \times 10^{-5}$ & - & & & & Amorph & & [47] \\
\hline & PLA- $\mathrm{LiClO}_{4}-\mathrm{EC}-\mathrm{SiO}_{2}$ & Solid & $1.3 \times 10^{-5}$ & - & - & - & - & Amorph & - & [46] \\
\hline & PLA/PHB/PC-LiPF 6 -EC & Gel & $1.5 \times 10^{-2}$ & - & 4.4 & - & 69 & Semi-cr & Battery & {$[48]$} \\
\hline & PLGA/SY-TBABF 4 & Solid & $10^{-10}$ & - & - & - & - & - & LEC & [372] \\
\hline & PLA-LiTFSI-Pyr ${ }_{14}$ TFSI & Solid & - & - & - & - & 0.1 & Amorph & Battery & [49] \\
\hline
\end{tabular}


Table 3. Cont.

\begin{tabular}{|c|c|c|c|c|c|c|c|c|c|c|}
\hline \multirow{2}{*}{ Polymer } & \multirow{2}{*}{ Electrolyte System } & \multirow[b]{2}{*}{ State } & \multicolumn{4}{|c|}{ Electrochemical Properties } & \multicolumn{2}{|c|}{ Physical Properties } & \multirow[b]{2}{*}{ Device } & \multirow[b]{2}{*}{ Ref } \\
\hline & & & $\sigma(\mathrm{s} / \mathrm{cm})$ & I-TN & Stability (V) & $E_{a}$ & $\mathrm{~T}_{\mathrm{g}}\left({ }^{\circ} \mathrm{C}\right)$ & Structural & & \\
\hline \multirow{4}{*}{$\begin{array}{l}\text { Vegetable oil-based } \\
\text { polyurethane }\end{array}$} & Palm-based PU- $\mathrm{LiCF}_{3} \mathrm{SO}_{3}$ & Solid & $1.6 \times 10^{-5}$ & - & - & - & - & Amorph & - & [8] \\
\hline & Palm-based PU-LiI- $\mathrm{I}_{2}-\mathrm{EC}$ & Solid & $7.6 \times 10^{-4}$ & - & - & $0.11 \mathrm{eV}$ & - & Amorph & DSSC & [51] \\
\hline & Castor oil-based PU-LiI & Solid & $1.4 \times 10^{-6}$ & 0.99 & 2.0 & $0.13 \mathrm{eV}$ & -27.5 & - & - & [52] \\
\hline & $\begin{array}{l}\text { Castor oil-based PU-NaI } \\
\text { Jatropha oil-based PU-LiClO } \\
\text {-EC }\end{array}$ & $\begin{array}{l}\text { Solid } \\
\text { Solid }\end{array}$ & $\begin{array}{l}4.3 \times 10^{-7} \\
1.3 \times 10^{-4}\end{array}$ & $\begin{array}{l}0.98 \\
0.83\end{array}$ & $\begin{array}{l}1.8 \\
4.8\end{array}$ & $\begin{array}{l}0.22 \mathrm{eV} \\
2.8 \mathrm{meV}\end{array}$ & -26.1 & $\stackrel{-}{\text { Amorph }}$ & - & [50] \\
\hline \multirow[b]{2}{*}{ Bacterial cellulose } & Bacterial cellulose-Nafion & Solid & $5.6 \times 10^{-2}$ & - & - & - & - & - & Fuel cell & [55] \\
\hline & Bacterial cellulose/PSSA-HCl & Solid & $10^{-3}$ & - & - & - & - & - & Fuel cell & [56] \\
\hline \multirow{9}{*}{ Gellan and Xanthan gum } & Gellan- $\mathrm{LiCF}_{3} \mathrm{SO}_{3}$ & Solid & $5.4 \times 10^{-4}$ & - & 5.4 & $14.6 \mathrm{~kJ} / \mathrm{mol}$ & - & Semi-cr & - & [58] \\
\hline & Gellan-LiI-Glycerol & Solid & $1.5 \times 10^{-3}$ & - & - & $2.4 \mathrm{~kJ} / \mathrm{mol}$ & - & - & - & [59] \\
\hline & Gellan-[ $\left[\mathrm{N}_{1112(\mathrm{OH})}\right]\left[\mathrm{NTf}_{2}\right]-\mathrm{Er}\left(\mathrm{CF}_{3} \mathrm{SO}_{3}\right)_{3}$ & Solid & $5.2 \times 10^{-6}$ & - & 3.5 & - & - & Semi-cr & ECD & [373] \\
\hline & Gellan-KI- $\mathrm{I}_{2}$ & Solid & $2.5 \times 10^{-2}$ & - & - & $0.24 \mathrm{eV}$ & - & Amorph & DSSC & [374] \\
\hline & Gellan-o- $\mathrm{H}_{3} \mathrm{PO}_{4}$ & & $5.1 \times 10^{-3}$ & - & - & $0.14 \mathrm{meV}$ & - & - & & \\
\hline & Gellan- $\mathrm{H}_{2} \mathrm{SO}_{4}$ & Gel & $1.5 \times 10^{-3}$ & - & - & $0.17 \mathrm{meV}$ & - & - & EDLC & [375] \\
\hline & Gellan-HCl & & $3.7 \times 10^{-4}$ & - & - & $0.19 \mathrm{meV}$ & - & - & & \\
\hline & Xanthan-PMII-I ${ }_{2}-\mathrm{TBP}-\mathrm{GSCN}$ & Gel & - & - & - & - & - & - & DSSC & [61] \\
\hline & $\begin{array}{l}\text { Xanthan- }-\mathrm{LiClO}_{4} \text {-glycerol } \\
\text { Xanthan- }-\mathrm{Li}_{1} \mathrm{~B}_{4} \mathrm{O}_{7} \text {-glycerol }\end{array}$ & Gel & $2.6 \times 10^{-3}$ & - & - & - & - & - & Super-capacitor & [23] \\
\hline
\end{tabular}

(I-TN: Ionic transference number; Ea: Activation energy, Tg: Glass transition temperature; Amorph: Amorphous; Semi-cr: Semi-crystalline). 


\subsection{Application of Bio-Based Polymer Electrolytes}

Bio-based polymer electrolytes have been tested in various electrochemical devices. This review focuses on the use of these electrolytes in batteries and DSSCs applications. Batteries have been widely used as energy supplies for portable devices, wearable electronics, and electric vehicles. Conventional batteries are made up of a cathode and anode, a separator to prevent physical contact between the two electrodes, and an electrolyte system. The most common anode materials used in batteries are titanium oxide, graphite, alloys, metal oxides, pure metal foils, etc. Meanwhile, the cathode materials used are vanadium oxide, molybdenum oxide, manganese oxide, silicates, $\mathrm{LiCoO}_{2}, \mathrm{LiFePO}_{4}$, $\mathrm{LiMn}_{2} \mathrm{O}_{4}$, etc. [376]. The electrolyte used in batteries' construction is typically organic liquid electrolytes. The electrolyte is one of the key components that determine the battery's performance which is related to the charging/discharging capacity, cycling performance, and current density [48]. In principle, when a battery is being charged, the ion moves from the cathode to the anode through the electrolyte, and during discharge, the ion will move back from the anode to the cathode. As discussed in the previous section, liquid electrolytes have a fundamental limitation for long-term operation due to their safety issues on evaporation and leakage, environmental concern, and restricted battery design. Hence, the best path is by replacing the conventional liquid electrolyte with all solid-state polymer electrolytes. Based on the literature, several studies on bio-based polymer electrolytes for lithium-ion batteries have been documented. The polymer systems include cellulose [146,155,160,161,165,169,177,181,182], chitosan [200,257], and natural rubber [360,367]. Despite the excellent performance, lithium-ion batteries rely on ultimately scarce and expensive resources. A sodium-ion battery is rather an interesting alternative as it is available abundantly at a very cheap cost. However, the use of bio-based polymer electrolytes in sodium-ion batteries is still in the early stage compared to lithium-ion batteries. A study on cellulose-based electrolyte on sodium-ion batteries was conducted by Colò and co-workers. The system shows good thermal stability and a wide electrochemical stability window [162]. Proton battery is another alternative to the lithium-ion battery that has been progressively studied. The electrochemical window for a proton battery is generally low, within the range of 1 to $2 \mathrm{~V}$. Despite that, the availability of low-cost proton conductors has made proton batteries a good alternative. Some studies on bio-based polymer electrolytes for proton batteries have been reported on cellulose $[129,133,179]$, chitosan [206,229,275], and natural rubber-based systems [350].

DSSC is the third generation of solar cells invented in 1991 by a team led by Gratzel [377]. Similarly to the previous generation of solar cells, DSSC converts sunlight energy directly into electrical energy through photovoltaic effects. DSSCs are interesting in regard to their remarkable advantages, such as low-cost production, robustness, colorful appearance, and possible flexibility. A typical DSSC consists of four components, which are a photoanode, a dye sensitizer, an electrolyte, and a counter electrode. The photoanode consists of a dye-coated nanocrystalline semiconductor oxide on a conducting substrate. It acts as a roadway for the electrons coursing through the cell. The counter electrode is usually a film of graphite or platinum. An electrolyte containing a redox couple fills the gap between the electrodes. The redox mediator is usually an organic solvent containing a redox system, such as an iodide/triiodide $\left(\mathrm{I}^{-} / \mathrm{I}^{3-}\right)$ couple $[378,379]$. The use of a bio-based polymer electrolyte in place of the conventional organic solvent electrolyte could solve the leakage, corrosion, and stability issues often caused by the liquid-type electrolyte. Based on the literature, the $\mathrm{I}^{-} / \mathrm{I}^{3-}$ is the most efficient and widely used redox couple for bio-based polymer electrolyte in DSSC. Various iodide salts have been tested, such as LiI, NaI, KI, $\mathrm{NH}_{4} \mathrm{I}$, etc. Some past study of bio-based polymer on DSSC is tabulated in Table 4. By far, the highest power conversion efficiency was achieved for cellulose-based electrolyte at $7.55 \%$ [139]. Despite this achievement, there are still some gaps that hinder the commercialization of bio-based polymer electrolytes in commercial application. Challenges that need to be addressed include the stability performance of the electrolyte overtime usage and the suitability of said materials in the selected application. 
Table 4. The photovoltaic performance of DSSC observed in bio-based polymers-salt matrix

\begin{tabular}{|c|c|c|c|c|c|c|c|c|}
\hline Polymer & Electrolyte System & State & $\sigma(\mathrm{s} / \mathrm{cm})$ & $\mathrm{JsC}\left(\mathrm{mA} / \mathrm{cm}^{2}\right)$ & $\mathrm{v}_{\mathrm{OC}}(\mathrm{V})$ & FF & $\eta(\%)$ & Ref. \\
\hline \multirow{5}{*}{ Starch } & Rice starch-LiI-MPII-TiO ${ }_{2}$ & Solid & $3.6 \times 10^{-4}$ & 0.49 & 0.45 & 0.75 & 0.17 & [88] \\
\hline & Rice starch-LiI & & $4.7 \times 10^{-5}$ & - & - & - & - & \\
\hline & Rice starch- $-\mathrm{NH}_{4} \mathrm{I}$ & Solid & $1.4 \times 10^{-4}$ & - & - & - & - & [96] \\
\hline & Rice starch-NaI & & $4.5 \times 10^{-4}$ & 2.40 & 0.48 & 0.67 & 0.78 & \\
\hline & Rice starch-NaI-MPII & Solid & $1.2 \times 10^{-3}$ & 4.78 & 0.57 & 0.76 & 2.09 & [97] \\
\hline \multirow{4}{*}{ Cellulose } & Cellulose-g-acrylic acid-[BmIm][I] & Gel & $7.3 \times 10^{-3}$ & 12.65 & 0.71 & 0.611 & 5.51 & [131] \\
\hline & CN-HPC-LiI- $\mathrm{I}_{2}-\mathrm{MHII}$ & Gel & $2.5 \times 10^{-3}$ & 14.40 & 0.76 & 0.70 & 7.55 & [139] \\
\hline & $\mathrm{PEO} / \mathrm{CMC}-\mathrm{NaI}-\mathrm{I}_{2}-\mathrm{MPII}$ & Gel & - & 10.03 & 0.75 & 0.69 & 5.18 & [147] \\
\hline & MFC/BEMA/PEGMA-NaI- $\mathrm{I}_{2}$ & Gel & - & 15.20 & 0.76 & 0.61 & 7.03 & [154] \\
\hline \multirow{2}{*}{ Chitosan } & Chitosan/PEO-NH 4 I & Solid & $3.7 \times 10^{-6}$ & 2.71 & 0.58 & 0.50 & 0.78 & [281] \\
\hline & N-phthaloyl chitosan-TPAI- $\mathrm{I}_{2}-\mathrm{EC}$ & Solid & $5.5 \times 10^{-3}$ & 12.72 & 0.60 & 0.66 & 5.00 & [284] \\
\hline \multirow{16}{*}{ Agar } & Agar-LiI- $\mathrm{I}_{2}-\mathrm{TiO}_{2}$ & Gel & - & 10.96 & 0.54 & 0.57 & 4.74 & [299] \\
\hline & Agar- $\mathrm{NH}_{4} \mathrm{I}-\mathrm{I}_{2}-$ Glycerol & Gel & $4.9 \times 10^{-3}$ & 0.007 & 0.29 & - & - & [380] \\
\hline & Agar-LiI- $\mathrm{I}_{2}-\mathrm{NiO}$ & Gel & - & - & - & - & 2.95 & [303] \\
\hline & Agar-MPII-PC-GuSCN-NMBI-I ${ }_{2}$ & & & 11.73 & 0.70 & 0.64 & 5.25 & \\
\hline & Agar-AEII-PC-GuSCN-NMBI-I ${ }_{2}$ & & - & 11.71 & 0.72 & 0.65 & 5.45 & [381] \\
\hline & Agar-DAII-PC-GuSCN-NMBI-I ${ }_{2}$ & Gel & - & 11.53 & 0.70 & 0.62 & 4.97 & {$[381]$} \\
\hline & Agar-APII-PC-GuSCN-NMBI-I ${ }_{2}$ & & & 11.84 & 0.70 & 0.60 & 4.96 & \\
\hline & Agar-LiI- $\mathrm{I}_{2}-\mathrm{Fe}_{3} \mathrm{O}_{4}-\mathrm{SDS}$ & & - & 3.18 & 0.66 & 0.62 & 1.29 & \\
\hline & Agar-LiI- $\mathrm{I}_{2}-\mathrm{Fe}_{3} \mathrm{O}_{4}-\mathrm{PVP}$ & Gel & - & 3.00 & 0.67 & 0.59 & 1.19 & [305] \\
\hline & Agar-LiI- $\mathrm{I}_{2}-\mathrm{Fe}_{3} \mathrm{O}_{4}-\mathrm{TW}-80$ & & $3.0 \times 10^{-3}$ & 5.00 & 0.70 & 0.53 & 1.83 & \\
\hline & Agar-KI- $\mathrm{I}_{2}$ & Gel & $9.0 \times 10^{-3}$ & 3.27 & 0.67 & 0.24 & 0.54 & [382] \\
\hline & Agar-LiI- $\mathrm{I}_{2}-\mathrm{TiO}_{2}$ & & $2.7 \times 10^{-3}$ & 5.28 & 0.61 & 0.55 & 1.71 & \\
\hline & Agar-LiI- $\mathrm{I}_{2}-\mathrm{Co}_{3} \mathrm{O}_{4}$ & Gel & $4.4 \times 10^{-3}$ & 7.24 & 0.63 & 0.46 & 2.11 & [308] \\
\hline & Agar-LiI- $\mathrm{I}_{2}-\mathrm{NiO}$ & & $3.3 \times 10^{-3}$ & 6.20 & 0.62 & 0.52 & 2.02 & \\
\hline & Agar- $\mathrm{Na}_{2} \mathrm{~S} / \mathrm{S}$-glycerol & Gel & - & 10.75 & 0.58 & 0.47 & 2.97 & [310] \\
\hline & Agar-KI-MPII & Gel & $1.5 \times 10^{-3}$ & 9.28 & 0.46 & 0.50 & 2.16 & [313] \\
\hline \multirow{5}{*}{ Carrageenan } & Carboxymethyl k-carrageenan/CMC- $\mathrm{NH}_{4} \mathrm{I}$ & Solid & $2.4 \times 10^{-3}$ & 0.49 & 0.60 & 0.64 & 0.13 & [316] \\
\hline & $\mathrm{K}$-carrageenan-TBAI- $\mathrm{I}_{2}-\mathrm{TiO}_{2}$ & & - & 1.83 & 0.75 & 0.55 & 0.76 & \\
\hline & k-carrageenan- $\mathrm{TBAI}-\mathrm{I}_{2}-\mathrm{Fe}_{2} \mathrm{O}_{3}$ & Solid & - & 3.96 & 0.68 & 0.07 & 0.20 & [317] \\
\hline & $\mathrm{K}$-carrageenan-TBAI-I $\mathrm{I}_{2}$-halloysite clay & & - & 1.39 & 0.74 & 0.50 & 0.51 & \\
\hline & Carboxymethyl k-carrageenan/CMC-LiI- $\mathrm{I}_{2}$ & Solid & $3.9 \times 10^{-3}$ & 0.40 & 0.49 & 0.57 & 0.11 & [318] \\
\hline \multirow{2}{*}{ Gelatine } & \multirow{2}{*}{$\begin{array}{l}\text { Poly(acrylic } \\
\text { acid-g-gelatine)/polypyrrole-KI- } \mathrm{I}_{2} \\
\text { Gelatine/Au-LiI- } \mathrm{I}_{2}\end{array}$} & Gel & $1.4 \times 10^{-2}$ & 2.76 & 0.66 & 0.70 & 1.28 & [330] \\
\hline & & Gel & $2.2 \times 10^{-2}$ & 4.94 & 0.65 & 0.60 & 1.97 & [342] \\
\hline \multirow{3}{*}{$\begin{array}{l}\text { Vegetable } \\
\text { oil-based } \\
\text { polyurethane }\end{array}$} & Palm-based PU-LiI- $\mathrm{I}_{2}-\mathrm{EC}$ & Solid & $7.6 \times 10^{-4}$ & 0.06 & 0.14 & - & - & [51] \\
\hline & Castor oil-based PU-NaI & Solid & $4.3 \times 10^{-7}$ & 3.60 & 0.49 & 0.46 & 0.80 & [383] \\
\hline & Palm-based PU-MPII & Gel & $9.1 \times 10^{-4}$ & 3.30 & 0.71 & 0.36 & 1.00 & [384] \\
\hline \multirow{2}{*}{$\begin{array}{l}\text { Gellan and } \\
\text { Xanthan gum }\end{array}$} & Gellan-KI-I ${ }_{2}$ & Solid & $2.5 \times 10^{-2}$ & 3.20 & 0.57 & 0.90 & 1.47 & [374] \\
\hline & Xanthan-PMII- $\mathrm{I}_{2}-\mathrm{TBP}-\mathrm{GSCN}$ & Gel & - & - & - & - & 4.78 & [61] \\
\hline
\end{tabular}

( $\sigma$ : Conductivity; JSC: Short-circuit current density; Voc: Open circuit potential; FF: Fill factor; $\eta$ : Efficiency) 


\section{Summary and Outlook}

Based on the literature available to date, a variety of bio-polymers have been explored by researchers, and the number of studies keeps on expanding, particularly over the last few decades. Similar to conventional petrochemical-based polymer electrolytes, bio-based polymers also suffer from low ionic conductivity when compared to liquid electrolytes. In fact, many studies have attempted to address this limitation. Researchers have suggested a number of ways to tackle this shortcoming by introducing fillers, plasticizers, and polymer blending methods. Nevertheless, the literature is still lacking in terms of the evaluation of the shelf life performance of bio-based electrolytes. This is another point of view that demands further investigation. As for applications, some of the bio-based polymer electrolytes have been tested in dye-sensitized solar cells, super-capacitors, and batteries. Despite the various types of bio-based materials that have been investigated as polymer electrolytes, they have yet to attain the status of commercial viability. Hence, extensive studies are still required to develop a system to achieve a level of performance that is comparable to the conventional liquid electrolytes. One interesting approach is to use computational and molecular modeling to understand the fundamental aspects of the materials. Such tools will provide important information, especially on the conduction mechanism, and can be used to assist and support the interpretation of experiments. Future work in this area will be very interesting as it will provide an in-depth understanding of the theoretical principle of the polymer electrolyte. From the preceding review, proper designs based on carefully selected materials and methods are expected to improve the bio-based polymer electrolytes performance.

Author Contributions: Conceptualization, M.M.A., M.R., L.C.A., M.S.S., A.A., and S.N.A.M.J., writing一review and editing, M.M.A., M.R., S.C.A., and M.S.S. All authors have read and agreed to the published version of the manuscript.

Funding: This research was funded by the MyBrain Scholarship, MOHE and the UPM Grant Putra-IPS (9493600).

Conflicts of Interest: The authors declare no conflicts of interest.

\section{Abbreviations}

$\begin{array}{ll}\mathrm{AEII} & \text { 1-allyl-3-ethylimidadolium iodide } \\ \mathrm{AgNO}_{3} & \text { Silver nitrate } \\ \mathrm{Al}_{2} \mathrm{O}_{3} & \text { Aluminium oxide } \\ \mathrm{Al}_{2} \mathrm{SiO}_{5} & \text { Aluminium silicate } \\ {[\mathrm{AmIm}][\mathrm{Cl}]} & \text { 1-allyl-3-methylimidazolium chloride } \\ \mathrm{APII} & \text { 1-allyl-3-propylimidazolium iodide } \\ \mathrm{BaTiO}_{3} & \text { Barium titanate } \\ \mathrm{BDG} & \text { Diethylene glycol dibutylether } \\ \mathrm{BEMA} & \text { Bisphenol A ethoxylate dimethacrylate } \\ \mathrm{BMATFSI} & \text { Butyl-trimethyl ammonium bis(trifluoromethylsulfonyl)imide } \\ {[\mathrm{BmIm}][\mathrm{Cl}]} & \text { 1-butyl-3-methylimidazolium chloride } \\ {[\mathrm{BmIm}][\mathrm{I}]} & \text { 1-butyl-3-methylimidazolium iodide } \\ {[\mathrm{BmIm}][\mathrm{OAc}]} & \text { 1-butyl-3-methylimidazoliumacetate } \\ {[\mathrm{BmIm}]\left[\mathrm{PF}{ }_{6}\right]} & \text { 1-butyl-3-methylimidazolium hexafluorophosphate } \\ {[\mathrm{BmIm}][\mathrm{Tf}]} & \text { 1-butyl-3-methylimidazolium trifluoromethanesulfonate } \\ {[\mathrm{BmIm}][\mathrm{TFSI}]} & \text { 1-butyl-3-methylimidazolium trifluoromethanesulfonyl imide } \\ \mathrm{Bu}_{4} \mathrm{NBF}_{4} & \text { Tetrabutylammonium tetrafluoroborat } \\ \mathrm{Ce}\left(\mathrm{CF} \mathrm{SO}_{3}\right)_{3} & \text { Cerium triflate } \\ {[\mathrm{Ch}][\mathrm{OAc}]} & \text { Trimethyl-ethanolammonium acetate } \\ \mathrm{CH}{ }_{3} \mathrm{COONH} & \text { Ammonium acetate } \\ \mathrm{CMC} & \text { Carboxymethyl cellulose } \\ \mathrm{CMCh} & \text { Carboxymethyl chitosan } \\ \mathrm{CNC} & \text { Cellulose nanocrystals } \\ \mathrm{CN}-\mathrm{HPC} & \text { Cyanoethylated hydroxypropyl cellulose } \\ & \end{array}$




\begin{tabular}{|c|c|}
\hline $\mathrm{Co}_{3} \mathrm{O}_{4}$ & Cobalt oxide \\
\hline DAII & 1-3-diallylimidazolium iodide \\
\hline DES & Deep eutectic solvent \\
\hline DMC & Dimethyl carbonate \\
\hline DPEO & Poly(ethylene oxice) diisocyanate \\
\hline DSSC & Dye sensitized solar cell \\
\hline DTAB & Dodecyltrimethyl ammonium bromide \\
\hline EC & Ethylene carbonate \\
\hline ECD & Electrochromic device \\
\hline EDLC & Electrical double layer capasitor \\
\hline EMC & Ethyl methyl carbonate \\
\hline$[\mathrm{EmIm}][\mathrm{Br}]$ & 1-butyl-3-methylimidazolium bromide \\
\hline$[\mathrm{EmIm}][\mathrm{Cl}]$ & 1-ethyl-3-methylimidazolium chloride \\
\hline$[\mathrm{EmIm}]\left[\mathrm{CF}_{3} \mathrm{SO}_{3}\right]$ & 2-ethyl-3-methylimidazolium trifluoromethanesulfonate \\
\hline$[\mathrm{EmIm}]\left[\mathrm{C}_{1} \mathrm{SO}_{3}\right]$ & 1-ethyl-3-methylimidazolium methylsulfonate \\
\hline$[\mathrm{EmIm}]\left[\mathrm{C}_{2} \mathrm{SO}_{3}\right]$ & 1-ethyl-3-methylimidazolium ethylsulfonate \\
\hline$[\mathrm{EmIm}]\left[\mathrm{C}_{4} \mathrm{SO}_{3}\right]$ & 1-ethyl-3-methylimidazolium butylsulfonate \\
\hline$[\mathrm{EmIm}]\left[\mathrm{C}_{2} \mathrm{SO}_{4}\right]$ & 1-ethyl-3-methylimidazolium ethylsulfate \\
\hline$[\mathrm{EmIm}]\left[\mathrm{Eu}(\mathrm{SCN})_{4}\right]$ & 1-ehtyl-3-methylimidazolium europium(III) tetrathiocyanate \\
\hline$[\mathrm{EmIm}][\mathrm{OAc}]$ & 1-ethyl-3-methylimidazolium acetate \\
\hline$[\mathrm{EmIm}]\left[\mathrm{N}(\mathrm{CN})_{2}\right]$ & 1-ethyl-3-methylimidazolium dicyanamide \\
\hline$[\mathrm{EmIm}]\left[\mathrm{NO}_{3}\right]$ & 1-ethyl-3-methylimidazolium nitrate \\
\hline$[\mathrm{EmIm}][\mathrm{SCN}]$ & 1-ethyl 3-methylimidazolium thiocyanate \\
\hline$[$ EmIm] $[$ TFSI] & 1-ethyl-3-methylimidazolium bis(trifluoromethylsulfonyl)imide \\
\hline ENR-25 & $25 \%$ epoxidized natural rubber \\
\hline ENR-50 & $50 \%$ epoxidized natural rubber \\
\hline EO-EPI & ethylene oxide-epichlorohydrin \\
\hline $\mathrm{Er}\left(\mathrm{CF}_{3} \mathrm{SO}_{3}\right)_{3}$ & Erbium triflate \\
\hline ES & Ethylene sulphite \\
\hline EtC & Ethyl carbonate \\
\hline$\left[\mathrm{Eu}(\mathrm{pic})_{3}\right]$ & Europium picrate \\
\hline $\mathrm{Eu}\left(\mathrm{CF}_{3} \mathrm{SO}_{3}\right)_{3}$ & Europium triflate \\
\hline $\mathrm{Fe}_{2} \mathrm{O}_{3}$ & Iron (III) oxide \\
\hline $\mathrm{Fe}_{3} \mathrm{O}_{4}$ & Iron (II,III) oxide \\
\hline GBL & $\gamma$-butyrolactone \\
\hline $\mathrm{GO}$ & Graphene oxide \\
\hline GSCN & Guanidinium thiocyanate \\
\hline $\mathrm{H}_{2} \mathrm{SO}_{4}$ & Sulphuric acid \\
\hline $\mathrm{HCF}$ & Hexacyanoferrate \\
\hline $\mathrm{HCl}$ & Hydrochloric acid \\
\hline HEC & Hydroxyethyl cellulose \\
\hline HPC & Hydroxypropyl cellulose \\
\hline HPMC & Hydroxypropyl methyl cellulose \\
\hline $\mathrm{I}_{2}$ & Iodine \\
\hline $\mathrm{KCl}$ & Potassium chloride \\
\hline KI & Potassium iodide \\
\hline LENR-50 & Liquid $50 \%$ epoxidized natural rubber \\
\hline $\mathrm{Li}_{2} \mathrm{~B}_{4} \mathrm{O}_{7}$ & Lithium tetraborate \\
\hline $\mathrm{LiBF}_{4}$ & Lithium tetrafluoroborate \\
\hline LiBoB & Lithium bis(oxalato) borate \\
\hline LiBs & lithium benzenesulfonate \\
\hline $\mathrm{LiCF}_{3} \mathrm{SO}_{3}$ & Lithium trifluoromethanesulfonate \\
\hline $\mathrm{LiCl}$ & Lithium chloride \\
\hline $\mathrm{LiClO}_{4}$ & Lithium perchlorate \\
\hline
\end{tabular}




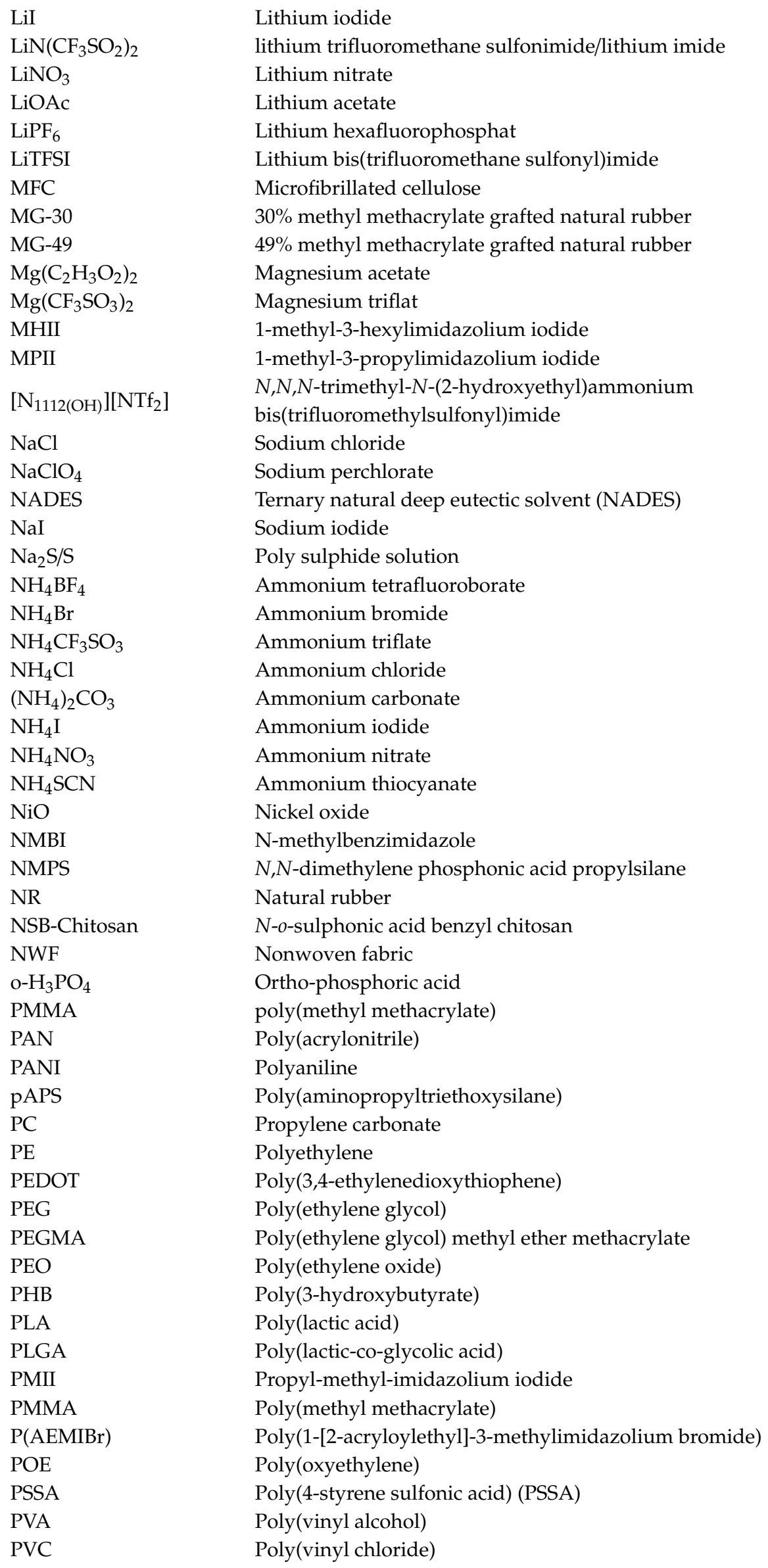




$\begin{array}{ll}\text { PVDF } & \text { Poly(vinylidene fluoride) } \\ \text { PVDF-HFP } & \text { Poly(vinylidene fluoride-hexafluoro propylene) } \\ \text { PVP } & \text { Poly(vinylpyrrolidone) } \\ \text { PVPA } & \text { poly(vinylphosphonic acid) } \\ \mathrm{Pyr}_{14} \text { TFSI } & \text { N-butyl-N-methylpyrrolidinium bis(trifluoromethane-sulfonyl) imide } \\ \mathrm{SDS} & \text { Sodium dodecyl sulphate } \\ \mathrm{SiO}_{2} & \text { Silicon diioxide } \\ \mathrm{SY} & \text { Super yellow } \\ \mathrm{TBABF} & \text { Tetrabutylammonium tetrafluorborate } \\ \mathrm{TBAI} & \text { Tetrabutylammonium iodide } \\ \mathrm{TBP} & \text { 4-tert-butylpyridine } \\ \mathrm{TEGDME} & \text { Tetra(ethylene) glycol dimethyl ether } \\ \mathrm{TiO}_{2} & \text { Titanium dioxide } \\ \mathrm{Tm}\left(\mathrm{CF}_{3} \mathrm{SO}_{3}\right)_{3} & \text { thulium triflate } \\ \mathrm{TPAI} & \text { Tetrapropylammonium iodide } \\ \mathrm{TW}-80 & \text { Polysorbate } 80 \\ \mathrm{Zn}\left(\mathrm{CF}_{3} \mathrm{SO}_{3}\right)_{2} & \text { Zinc triflate }\end{array}$

\section{References}

1. Tanase, E.E.; Rapa, M.; Popa, S. Biopolymers based on renewable resources-A review. Sci. Bull. Ser. F Biotechnol. 2014, 18, 188-195.

2. Malhotra, B.; Keshwani, A.; Kharkwal, H. Natural polymer based cling films for food packaging. Int. J. Pharm. Pharm. Sci. 2015, 7, 10-18.

3. Imam, S.H.; Bilbao-Sainz, C.; Chiou, B.; Glenn, G.M.; Orts, W.J. Biobased adhesives, gums, emulsions and binders: Current trends and future prospects. J. Adhes. Sci. Technol. 2012, 27, 1-26. [CrossRef]

4. Kucharski, M.; Łukaszewicz, T.; Mrozek, P. New electrolyte for electrochromic devices. Opto-Electron. Rev. 2004, 12, 175-180.

5. Hallinan, D.T., Jr.; Balsara, N.P. Polymer electrolytes. Annu. Rev. Mater Res 2013, 43, 503-525. [CrossRef]

6. Varshney, P.K.; Gupta, S. Natural polymer-based electrolytes for electrochemical devices: A review. Ionics 2011, 17, 479-483. [CrossRef]

7. Fenton, D.E.; Parker, J.M.; Wright, P.V. Complexes of alkali metal ions with poly(ethylene oxide). Polymer 1973, 14, 589. [CrossRef]

8. Daud, F.N.; Ahmad, A.; Badri, K.H. An investigation on the properties of palm-based polyurethane solid polymer electrolyte. Int. J. Polym. Sci. 2014, 2014, 1-5. [CrossRef]

9. Aziz, S.B.; Woo, T.J.; Kadir, M.F.Z.; Ahmed, H.M. A conceptual review on polymer electrolytes and ion transport models. J. Sci. Adv. Mater. Devices 2018, 3, 1-17. [CrossRef]

10. Liew, C.W.; Ramesh, S. Studies on ionic liquid-based corn starch biopolymer electrolytes coupling with high ionic transport number. Cellulose 2013, 20, 3227-3237. [CrossRef]

11. Raphael, E.; Avellaneda, C.O.; Manzolli, B.; Pawlicka, A. Agar-based films for application as polymer electrolytes. Electrochim. Acta 2010, 55, 1455-1459. [CrossRef]

12. Khiar, A.S.A.; Arof, A.K. Conductivity studies of starch-based polymer electrolytes. Ionics 2010, 16, 123-129. [CrossRef]

13. Kumar, M.; Tiwari, T.; Srivastava, N. Electrical transport behaviour of bio-polymer electrolyte system: Potato starch + ammonium iodide. Carbohydr. Polym. 2012, 88, 54-60. [CrossRef]

14. Pawlicka, A.; Machado, G.O.; Guimaraes, K.V.; Dragunski, D.C. Solid polymeric electrolytes obtained from modified natural polymers. Proc. SPIE Int. Soc. Opt. Eng. 2002, 5136, $274-279$.

15. Gandini, A.; Lacerda, T.M. From monomers to polymers from renewable resources: Recent advances. Prog. Polym. Sci. 2015, 48, 1-39. [CrossRef]

16. Yahya, M.Z.A.; Arof, A.K. Effect of oleic acid plasticizer on chitosan-lithium acetate solid polymer electrolytes. Eur. Polym. J. 2003, 39, 897-902. [CrossRef]

17. Majid, S.R.; Arof, A.K. Proton-conducting polymer electrolyte films based on chitosan acetate complexed with $\mathrm{NH}_{4} \mathrm{NO}_{3}$ salt. Phys. B Condens. Matter 2005, 355, 78-82. [CrossRef] 
18. Buraidah, M.H.; Teo, L.P.; Majid, S.R.; Arof, A.K. Ionic conductivity by correlated barrier hopping in $\mathrm{NH}_{4} \mathrm{I}$ doped chitosan solid electrolyte. Phys. B Condens. Matter 2009, 404, 1373-1379. [CrossRef]

19. Nomanbhay, S.M.; Palanisamy, K. Removal of heavy metal from industrial wastewater using chitosan coated oil palm shell charcoal. Electron. J. Biotechnol. 2005, 8, 43-53. [CrossRef]

20. Tripathi, S.; Mehrotra, G.K.; Dutta, P.K. Physicochemical and bioactivity of cross-linked chitosan-PVA film for food packaging applications. Int. J. Biol. Macromol. 2009, 45, 372-376. [CrossRef]

21. Osman, Z.; Ibrahim, Z.A.; Arof, A.K. Conductivity enhancement due to ion dissociation in plasticized chitosan based polymer electrolytes. Carbohydr. Polym. 2001, 44, 167-173. [CrossRef]

22. Mobarak, N.N.; Jumaah, F.N.; Ghani, M.A.; Abdullah, M.P.; Ahmad, A. Carboxymethyl carrageenan based biopolymer electrolytes. Electrochim. Acta 2015, 175, 224-231. [CrossRef]

23. Sudhakar, Y.N.; Selvakumar, M.; Krishna Bhat, D. Lithium salts doped biodegradable gel polymer electrolytes for supercapacitor application. J. Mater. Environ. Sci. 2015, 6, 1218-1227.

24. BeMiller, J.N. Gums and related polysaccharides. In Glycoscience: Chemistry and Chemical Biology, 2nd ed.; Fraser-Reid, B.O., Tatsuta, K., Thiem, J., Eds.; Springer: Berlin/Heidelberg, Germany, 2009; pp. 1514-1533.

25. Nwanya, A.C.; Amaechi, C.I.; Udounwa, A.E.; Osuji, R.U.; Maaza, M.; Ezema, F.I. Complex impedance and conductivity of agar-based ion-conducting polymer electrolytes. Appl. Phys. A 2015, 119, 387-396. [CrossRef]

26. Raphael, E.; Avellaneda, C.O.; Aegerter, M.A.; Silva, M.M. Agar-based gel electrolyte for electrochromic device application. Mol. Cryst. Liq. Cryst. 2012, 554, 264-272. [CrossRef]

27. Kasem, K.K. Electrochemical behavior of some redox systems pendant in agar gel. J. New Mater. Electrochem. Syst. 2005, 8, 189-195.

28. Rudhziah, S.; Ahmad, A.; Ahmad, I.; Mohamed, N.S. Biopolymer electrolytes based on blend of kappa-carrageenan and cellulose derivatives for potential application in dye sensitized solar cell. Electrochim. Acta 2015, 175, 162-168. [CrossRef]

29. Mobarak, N.N.; Ramli, N.; Ahmad, A.; Rahman, M.Y.A. Chemical interaction and conductivity of carboxymethyl K-carrageenan based green polymer electrolyte. Solid State Ion. 2012, 224, 51-57. [CrossRef]

30. Rudhziah, S.; Rani, M.S.A.; Ahmad, A.; Mohamed, N.S.; Kaddami, H. Potential of blend of K-carrageenan and cellulose derivatives for green polymer electrolyte application. Ind. Crop. Prod. 2015, 72, 133-141. [CrossRef]

31. Endreû, H.; Neuenbu, F.K.G.P.; Christensen, S.H.; Aps, C.P.K. Pectins. In Handbook of Hyrdocolloids, 2nd ed.; Phillips, G.O., Williams, P.A., Eds.; Woodhead Publishing Limited: Sawston, UK, 2009; pp. 274-297.

32. Smith, D. Jams and preserves. Methods of manufacture. In Encyclopedia of Food Sciences and Nutrition, 2nd ed.; Caballero, B., Ed.; Academic Press: Cambridge, MA, USA, 2003; pp. 3409-3415.

33. Andrade, J.R.; Raphael, E.; Pawlicka, A. Plasticized pectin-based gel electrolytes. Electrochim. Acta 2009, 54, 6479-6483. [CrossRef]

34. Thombare, N.; Jha, U.; Mishra, S.; Siddiqui, M.Z. Guar gum as a promising starting material for diverse applications: A review. Int. J. Biol. Macromol. 2016, 88, 361-372. [CrossRef] [PubMed]

35. Del Agua, I.; Mantione, D.; Casado, N.; Sanchez-Sanchez, A.; Malliaras, G.G.; Mecerreyes, D. Conducting polymer iongels based on PEDOT and guar gum. ACS Macro Lett. 2017, 6, 473-478. [CrossRef]

36. Mudgil, D.; Barak, S.; Khatkar, B.S. Guar gum: Processing, properties and food applications-A review. J. Food Sci. Technol. 2014, 51, 409-418. [CrossRef]

37. Sudhakar, Y.N.; Selvakumar, M.; Bhat, D.K. Tubular array, dielectric, conductivity and electrochemical properties of biodegradable gel polymer electrolyte. Mater. Sci. Eng. B Solid-State Mater. Adv. Technol. 2014, 180, 12-19. [CrossRef]

38. Zhang, B.; Sudre, G.; Quintard, G.; Serghei, A.; David, L.; Bernard, J.; Fleury, E.; Charlot, A. Guar gum as biosourced building block to generate highly conductive and elastic ionogels with poly(ionic liquid) and ionic liquid. Carbohydr. Polym. 2017, 157, 586-595. [CrossRef]

39. Alves, R.; Silva, M.M. The influence of glycerol and formaldehyde in gelatin-based polymer electrolytes. Mol. Cryst. Liq. Cryst. 2014, 591, 64-73. [CrossRef]

40. Haug, I.J.; Draget, K.I. Gelatin. In Handbook of Hyrdocolloidsal, 2nd ed.; Phillips, G.O., Williams, P.A., Eds.; Woodhead Publishing: Sawston, UK, 2009; pp. 142-163.

41. Pawlicka, A.; Firmino, A.; Vieira, D.; Sentanin, F.; Grote, J.G.; Kajzar, F. Gelatin- and DNA-based ionic conducting membranes for electrochromic devices. Proc. SPIE 2009, 7487, 1-12.

42. Vieira, D.F.; Avellaneda, O.; Pawlicka, A. Conductivity study of a gelatin-based polymer electrolyte. Electrochim. Acta 2007, 53, 1404-1408. [CrossRef] 
43. Kurian, T.; Mathew, N.M. Natural rubber: Production, properties and applications. In Biopolymers: Biomedical and Environmental Applications; Kalia, S., Avérous, L., Eds.; Scrivener Publishing: Beverly, MA, USA, 2011; pp. 403-436.

44. Yoshizawa, M.; Marwanta, E.; Ohno, H. Preparation and characteristics of natural rubber/poly(ethylene oxide) salt hybrid mixtures as novel polymer electrolytes. Polymer 2000, 41, 9049-9053. [CrossRef]

45. Ahmad,H.A.; Ismail, H.; Rashid, A.A. ENR-50 Compatibilized natural rubber/recycled acrylonitrile-butadiene rubber blends. Sains Malays. 2015, 44, 835-842. [CrossRef]

46. Chew, K.W.; Ng, T.C.; How, Z.H. Conductivity and microstructure study of PLA-based polymer electrolyte salted with lithium perchloride, $\mathrm{LiClO}_{4}$. Int. J. Electrochem. Sci. 2013, 8, 6354-6364.

47. Chew, K.W. Development of novel bio-degradable electrolyte based on polylactide (PLA) for lithium rechargeable battery. Adv. Mater. Res. 2013, 853, 270-275. [CrossRef]

48. Osinska-Broniarz, M.; Gieparda, W.; Wesole, D.; Martyla, A.; Przekop, R.; Sierczynska, A. Preparation and characterization of gel polymer electrolytes based on electrospun PLA/PHB membranes for lithium-ion batteries. ECS Trans. 2015, 70, 79-88. [CrossRef]

49. Osada, I.; Hosseini, S.M.; Jeong, S.; Passerini, S. Novel ternary polymer electrolytes based on poly(lactic acid) from sustainable sources. ChemElectroChem 2017, 4, 463-467. [CrossRef]

50. Mustapa, S.R.; Aung, M.M.; Ahmad, A.; Mansor, A.; TianKhoon, L. Preparation and characterization of jatropha oil-based polyurethane as non-aqueous solid polymer electrolyte for electrochemical devices. Electrochim. Acta 2016, 222, 293-302. [CrossRef]

51. Su'ait, M.S.; Ahmad, A.; Badri, K.H.; Mohamed, N.S.; Rahman, M.Y.A.; Azanza Ricardo, C.L.; Scardi, P. The potential of polyurethane bio-based solid polymer electrolyte for photoelectrochemical cell application. Int. J. Hydrogen Energy 2014, 39, 3005-3017. [CrossRef]

52. Ibrahim, S.; Ahmad, A.; Mohamed, N.S. Characterization of novel castor oil-based polyurethane polymer electrolytes. Polymers 2015, 7, 747-759. [CrossRef]

53. Esa, F.; Tasirin, S.M.; Rahman, N.A. Overview of bacterial cellulose production and application. Agric. Agric. Sci. Procedia 2014, 2, 113-119. [CrossRef]

54. Park, J.K.; Jung, J.Y.; Khan, T. Bacterial Cellulose; Woodhead Publishing Limited: Sawston, UK, 2009.

55. Jiang, G.P.; Zhang, J.; Qiao, J.L.; Jiang, Y.M.; Zarrin, H.; Chen, Z.; Hong, F. Bacterial nanocellulose/Nafion composite membranes for low temperature polymer electrolyte fuel cells. J. Power Sources 2015, 273, 697-706. [CrossRef]

56. Gadim, T.D.O.; Loureiro, F.J.A.; Vilela, C.; Rosero-Navarro, N.; Silvestre, A.J.D.; Freire, C.S.R.; Figueiredo, F.M.L. Protonic conductivity and fuel cell tests of nanocomposite membranes based on bacterial cellulose. Electrochim. Acta 2017, 233, 52-61. [CrossRef]

57. Sworn, G. Gellan gum. In Handbook of Hyrdocolloidsal, 2nd ed.; Phillips, G.O., Williams, P.A., Eds.; Woodhead Publishing: Sawston, UK, 2009; pp. 204-227.

58. Noor, I.S.M.; Majid, S.R.; Arof, A.K.; Djurado, D.; Claro Neto, S.; Pawlicka, A. Characteristics of gellan gum-LiCF $\mathrm{SO}_{3}$ polymer electrolytes. Solid State Ion. 2012, 225, 649-653. [CrossRef]

59. Halim, N.F.A.; Majid, S.R.; Arof, A.K.; Kajzar, F.; Pawlicka, A. Gellan gum-LiI gel polymer electrolytes. Mol. Cryst. Liq. Cryst. 2012, 554, 232-238. [CrossRef]

60. Sworn, G. Xanthan gum. In Handbook of Hyrdocolloidsal, 2nd ed.; Phillips, G.O., Williams, P.A., Eds.; Woodhead Publishing: Sawston, UK, 2009; pp. 186-203.

61. Park, S.J.; Yoo, K.; Kim, J.-Y.; Kim, J.Y.; Lee, D.-K.; Kim, B.; Kim, H.; Kim, J.H.; Cho, J.; Ko, M.J. Water-based thixotropic polymer gel electrolyte for dye-sensitized solar cells. ACS Nano 2013, 7, 4050-4056. [CrossRef] [PubMed]

62. Gaikwad, U.V.; Pande, S.A. A review of biopolymer chitosan blends in polymer system. Int. Res. J. Sci. Eng. 2013, 1, 13-16.

63. Ramly, K.; Isa, M.I.N.; Khiar, A.S.A. Conductivity and dielectric behaviour studies of starch/PEO $+x$ wt- $\% \mathrm{NH}_{4} \mathrm{NO}_{3}$ polymer electrolyte. Mater. Res. Innov. 2011, 15, 82-85. [CrossRef]

64. Shukur, M.F.; Ithnin, R.; Illias, H.A.; Kadir, M.F.Z. Proton conducting polymer electrolyte based on plasticized chitosan-PEO blend and application in electrochemical devices. Opt. Mater. 2013, 35, 1834-1841. [CrossRef]

65. Abdullah, D.J.S.A.; Alcázar, J.B.; Barbosa, C.V.; Carreño, N.L.V.; Avellaneda, C.S.O. Influence of the $\mathrm{NiO}$ nanoparticles on the ionic conductivity of the agar-based electrolyte. Ciência e Tecnologia 2014, 24, 8-12. 
66. Lopes, L.V.S.; Dragunski, D.C.; Pawlicka, A.; Donoso, J.P. Nuclear magnetic resonance and conductivity study of starch based polymer electrolytes. Electrochim. Acta 2003, 48, 2021-2027. [CrossRef]

67. Mattos, R.I.; Pawlicka, A.; Tambelli, C.E.; Donoso, J.P. NMR and conductivity study of strach-based polymer electrolytes. Mol. Cryst. Liq. Cryst. 2006, 447, 55-64. [CrossRef]

68. Ma, X.; Yu, J.; He, K.; Wang, N. The effects of different plasticizers on the properties of thermoplastic starch as solid polymer electrolytes. Macromol. Mater. Eng. 2007, 292, 503-510. [CrossRef]

69. Pawlicka, A.; Sabadini, A.; Raphael, E.; Dragunski, D. Ionic conductivity thermogravimetry measurements of starch-based polymeric electrolytes. Mol. Cryst. Liq. Cryst. 2008, 485, 804-816. [CrossRef]

70. Marcondes, R.F.M.S.; D'Agostini, P.S.; Ferreira, J.; Girotto, E.M.; Pawlicka, A.; Dragunski, D.C. Amylopectin-rich starch plasticized with glycerol for polymer electrolyte application. Solid State Ion. 2010, 181, 586-591. [CrossRef]

71. Ramesh, S.; Shanti, R.; Morris, E.; Durairaj, R. Utilisation of corn starch in production of 'green' polymer electrolytes. Mater. Res. Innov. 2011, 15, 8-13. [CrossRef]

72. Ramesh, S.; Liew, C.-W.; Arof, A.K. Ion conducting corn starch biopolymer electrolytes doped with ionic liquid 1-butyl-3-methylimidazolium hexafluorophosphate. J. Non. Cryst. Solids 2011, 357, 3654-3660. [CrossRef]

73. Tiwari, T.; Pandey, K.; Srivastava, N.; Srivastava, P.C. Effects of glutalraldehyde on electrical properties of arrowroot starch $+\mathrm{NaI}$ electrolyte system. Polym. Polym. Compos. 2011, 121, 1-7.

74. Liew, C.W.; Ramesh, S.; Ramesh, K.; Arof, A.K. Preparation and characterization of lithium ion conducting ionic liquid-based biodegradable corn starch polymer electrolytes. J. Solid State Electrochem. 2012, 16, 1869-1875. [CrossRef]

75. Samsudin, A.S.; Aziz, M.I.A.; Isa, M.I.N. Natural polymer electrolyte system based on sago: Structural and transport behavior characteristics. Int. J. Polym. Anal. Charact. 2012, 17, 600-607. [CrossRef]

76. Teoh, K.H.; Ramesh, S.; Arof, A.K. Investigation on the effect of nanosilica towards corn starch-lithium perchlorate-based polymer electrolytes. J. Solid State Electrochem. 2012, 16, 3165-3170. [CrossRef]

77. Ramesh, S.; Shanti, R.; Morris, E. Studies on the thermal behavior of CS:LiTFSI:[Amim] Cl polymer electrolytes exerted by different [Amim] Cl content. Solid State Sci. 2012, 14, 182-186. [CrossRef]

78. Ramesh, S.; Shanti, R.; Morris, E. Studies on the plasticization efficiency of deep eutectic solvent in suppressing the crystallinity of corn starch based polymer electrolytes. Carbohydr. Polym. 2012, 87, 701-706. [CrossRef]

79. Sudhakar, Y.N.; Selvakumar, M. Lithium perchlorate doped plasticized chitosan and starch blend as biodegradable polymer electrolyte for supercapacitors. Electrochim. Acta 2012, 78, 398-405. [CrossRef]

80. Shukur, M.F.; Ithnin, R.; Sonsudin, F.; Yahya, R.; Ahmad, Z.; Kadir, M.F.Z. Conduction mechanism and dielectric properties of solid biopolymer electrolyte incorporated with silver nitrate. Adv. Mater. Res. 2013, 701, 115-119. [CrossRef]

81. Shukur, M.F.; Ibrahim, F.M.; Majid, N.A.; Ithnin, R.; Kadir, M.F.Z. Electrical analysis of amorphous corn starch-based polymer electrolyte membranes doped with LiI. Phys. Scr. 2013, 88, 1-9. [CrossRef]

82. Shukur, M.F.; Sonsudin, F.; Yahya, R.; Ahmad, Z.; Ithnin, R. Electrical properties of starch based silver ion conducting solid biopolymer electrolyte. Adv. Mater. Res. 2013, 701, 120-124. [CrossRef]

83. Khanmirzaei, M.H.; Ramesh, S. Ionic transport and FTIR properties of lithium iodide doped biodegradable rice starch based polymer electrolytes. Int. J. Electrochem. Sci. 2013, 8, 9977-9991.

84. Sudhakar, Y.N.; Selvakumar, M. Ionic conductivity studies and dielectric studies of poly(styrene sulphonic acid)/starch blend polymer electrolyte containing $\mathrm{LiClO}_{4}$. J. Appl. Electrochem. 2013, 43, 21-29. [CrossRef]

85. Tiwari, T.; Kumar, M.; Srivastava, N.; Srivastava, P.C. Electrical transport study of potato starch-based electrolyte system-II. Mater. Sci. Eng. B Solid-State Mater. Adv. Technol. 2014, 182, 6-13. [CrossRef]

86. Shukur, M.F.; Ithnin, R.; Kadir, M.F.Z. Electrical characterization of corn starch-LiOAc electrolytes and application in electrochemical double layer capacitor. Electrochim. Acta 2014, 136, 204-216. [CrossRef]

87. Singh, R.; Baghel, J.; Shukla, S.; Bhattacharya, B.; Rhee, H.-W.; Singh, P.K. Detailed electrical measurements on sago starch biopolymer solid electrolyte. Phase Transit. 2014, 87, 1237-1245. [CrossRef]

88. Khanmirzaei, M.H.; Ramesh, S. Nanocomposite polymer electrolyte based on rice starch/ionic liquid/TiO 2 nanoparticles for solar cell application. Measurement 2014, 58, 68-72. [CrossRef]

89. Liew, C.W.; Ramesh, S. Comparing triflate and hexafluorophosphate anions of ionic liquids in polymer electrolytes for supercapacitor applications. Materials 2014, 7, 4019-4033. [CrossRef] 
90. Teoh, K.H.; Lim, C.-S.; Ramesh, S. Lithium ion conduction in corn starch based solid polymer electrolytes. Measurement 2014, 48, 87-95. [CrossRef]

91. Yusof, Y.M.; Majid, N.A.; Kasmani, R.M.; Illias, H.A.; Kadir, M.F.Z. The effect of plasticization on conductivity and other properties of starch/chitosan blend biopolymer electrolyte incorporated with ammonium iodide. Mol. Cryst. Liq. Cryst. 2014, 603, 73-88. [CrossRef]

92. Yusof, Y.M.; Shukur, M.F.; Illias, H.A.; Kadir, M.F.Z. Conductivity and electrical properties of corn starch-chitosan blend biopolymer electrolyte incorporated with ammonium iodide. Phys. Scr. 2014, 89, 1-11. [CrossRef]

93. Shukur, M.F.; Ithnin, R.; Kadir, M.F.Z. Electrical properties of proton conducting solid biopolymer electrolytes based on starch-chitosan blend. Ionics 2014, 20, 977-999. [CrossRef]

94. Shukur, M.F.; Ithnin, R.; Kadir, M.F.Z. Protonic transport analysis of starch-chitosan blend based electrolytes and application in electrochemical device. Mol. Cryst. Liq. Cryst. 2014, 603, 52-65. [CrossRef]

95. Teoh, K.H.; Lim, C.-S.; Liew, C.-W.; Ramesh, S. Electric double-layer capacitors with corn starch-based biopolymer electrolytes incorporating silica as filler. Ionics 2015, 21, 2061-2068. [CrossRef]

96. Khanmirzaei, M.H.; Ramesh, S.; Ramesh, K. Effect of different iodide salts on ionic conductivity and structural and thermal behavior of rice-starch-based polymer electrolytes for dye-sensitized solar cell application. Ionics 2015, 21, 2383-2391. [CrossRef]

97. Khanmirzaei, M.H.; Ramesh, S.; Ramesh, K. Polymer electrolyte based dye-sensitized solar cell with rice starch and 1-methyl-3-propylimidazolium iodide ionic liquid. Mater. Des. 2015, 85, 833-837. [CrossRef]

98. Shukur, M.F.; Kadir, M.F.Z. Electrical and transport properties of $\mathrm{NH}_{4} \mathrm{Br}$-doped cornstarch-based solid biopolymer electrolyte. Ionics 2015, 21, 111-124. [CrossRef]

99. Liew, C.W.; Ramesh, S. Electrical, structural, thermal and electrochemical properties of corn starch-based biopolymer electrolytes. Carbohydr. Polym. 2015, 124, 222-228. [CrossRef]

100. Chatterjee, B.; Kulshrestha, N.; Gupta, P.N. Electrical properties of starch-PVA biodegradable polymer blend. Phys. Scr. 2015, 90, 1-9. [CrossRef]

101. Navaratnam, S.; Sanusi, A.; Ahmad, A.H.; Ramesh, S.; Ramesh, K.; Othman, N. Conductivity studies of biopolymer electrolyte based on potato starch/chitosan blend doped with $\mathrm{LICF}_{3} \mathrm{SO}_{3}$. J. Teknol. 2015, 7, 1-5. [CrossRef]

102. Amran, N.N.A.; Manan, N.S.A.; Kadir, M.F.Z. The effect of $\mathrm{LiCF}_{3} \mathrm{SO}_{3}$ on the complexation with potato starch-chitosan blend polymer electrolytes. Ionics 2016, 22, 1647-1658. [CrossRef]

103. Teoh, K.H.; Lim, C.S.; Liew, C.W.; Ramesh, S. Preparation and performance analysis of barium titanate incorporated in corn starch-based polymer electrolytes for electric double layer capacitor application. J. Appl. Polym. Sci. 2016, 133, 1-8. [CrossRef]

104. Lin, Y.; Li, J.; Liu, K.; Liu, Y.; Liu, J.; Wang, X. Unique starch polymer electrolyte for high capacity all-solid-state lithium sulfur battery. Green Chem. 2016, 18, 3796-3803. [CrossRef]

105. Shukur, M.F.; Ithnin, R.; Kadir, M.F.Z. Ionic conductivity and dielectric properties of potato starch-magnesium acetate biopolymer electrolytes: The effect of glycerol and 1-butyl-3-methylimidazolium chloride. Ionics 2016, 22, 1113-1123. [CrossRef]

106. Chatterjee, B.; Kulshrestha, N.; Gupta, P.N. Nano composite solid polymer electrolytes based on biodegradable polymers starch and poly vinyl alcohol. Measurement 2016, 82, 490-499. [CrossRef]

107. Yusof, Y.M.; Kadir, M.F.Z. Electrochemical characterizations and the effect of glycerol in biopolymer electrolytes based on methylcellulose-potato starch blend. Mol. Cryst. Liq. Cryst. 2016, 627, 220-233. [CrossRef]

108. Kulshrestha, N.; Gupta, P.N. Structural and electrical characterizations of 50:50 PVA:starch blend complexed with ammonium thiocyanate. Ionics 2016, 22, 671-681. [CrossRef]

109. Khiar, A.S.A.; Anuar, M.R.S.; Md Parid, M.A. Effect of 1-ethyl-3-methylimidazolium nitrate on the electrical properties of starch/chitosan blend polymer electrolyte. Mater. Sci. Forum 2016, 846, 510-516. [CrossRef]

110. Railanmaa, A.; Lehtimäki, S.; Lupo, D. Comparison of starch and gelatin hydrogels for non-toxic supercapacitor electrolytes. Appl. Phys. A Mater. Sci. Process. 2017, 123, 1-8. [CrossRef]

111. Vernon-Carter, E.J.; Alvarez-Ramirez, J.; Bello-Perez, L.A.; Roldan-Cruz, C.; Garcia-Hernandez, A.; Huerta, L. The order of addition of corn starch/lithium perchlorate/glycerol affects the optical, mechanical, electrical properties of a solid polymer electrolyte. Ionics 2017, 23, 1-13. [CrossRef] 
112. Azli, A.A.; Manan, N.S.A.; Kadir, M.F.Z. The development of Li+ conducting polymer electrolyte based on potato starch/graphene oxide blend. Ionics 2017, 23, 411-425. [CrossRef]

113. Chauhan, J.K.; Kumar, M.; Yadav, M.; Tiwari, T.; Srivastava, N. Effect of $\mathrm{NaClO}_{4}$ concentration on electrolytic behaviour of corn starch film for supercapacitor application. Ionics 2017, 23, 2943-2949. [CrossRef]

114. Selvanathan, V.; Azzahari, A.D.; Adyani, A.A.; Yahya, R. Ternary natural deep eutectic solvent (NADES) infused phthaloyl starch as cost efficient quasi-solid gel polymer electrolyte. Carbohydr. Polym. 2017, 167, 210-218. [CrossRef]

115. Hamsan, M.H.; Shukur, M.F.; Kadir, M.F.Z. The effect of $\mathrm{NH}_{4} \mathrm{NO}_{3}$ towards the conductivity enhancement and electrical behavior in methyl cellulose-starch blend based ionic conductors. Ionics 2017, 23, 1137-1154. [CrossRef]

116. Hamsan, M.H.; Shukur, M.F.; Kadir, M.F.Z. $\mathrm{NH}_{4} \mathrm{NO}_{3}$ as charge carrier contributor in glycerolized potato starch-methyl cellulose blend-based polymer electrolyte and the application in electrochemical double-layer capacitor. Ionics 2017, 23, 1-25. [CrossRef]

117. Regiani, A.M.; De Oliveira-Machado, G.; LeNest, J.F.; Gandini, A.; Pawlicka, A. Cellulose derivatives as solid electrolyte matrixes. Macromol. Symp. 2001, 175, 45-53. [CrossRef]

118. Tambelli, C.E.; Donoso, J.P.; Regiani, A.M.; Pawlicka, A.; Gandini, A.; LeNest, J.F. Nuclear magnetic resonance and conductivity study of HEC/polyether-based polymer electrolytes. Electrochim. Acta 2001, 46, 1665-1672. [CrossRef]

119. Yue, Z.; McEwen, I.J.; Cowie, J.M.G. Ion conducting behaviour and morphology of solid polymer electrolytes based on a regioselectively substituted cellulose ether with PEO side chains. J. Mater. Chem. 2002, 12, 2281-2285. [CrossRef]

120. Yue, Z.; McEwen, I.J.; Cowie, J.M.G. Novel gel polymer electrolytes based on a cellulose ester with PEO side chains. Solid State Ion. 2003, 156, 155-162. [CrossRef]

121. Schroers, M.; Kokil, A.; Weder, C. Solid polymer electrolytes based on nanocomposites of ethylene oxide-epichlorohydrin copolymers and cellulose whiskers. J. Appl. Polym. Sci. 2004, 93, 2883-2888. [CrossRef]

122. Azizi Samir, M.A.S.; Mateos, A.M.; Alloin, F.; Sanchez, J.Y.; Dufresne, A. Plasticized nanocomposite polymer electrolytes based on poly(oxyethylene) and cellulose whiskers. Electrochim. Acta 2004, 49, 4667-4677. [CrossRef]

123. Machado, G.O.; Pawlicka, A.; Yonashiro, M. Solid polymeric electrolytes networks of hydroxypropyl cellulose with jeffamine. Nonlinear Opt. Quantum Opt. 2004, 32, 141-148.

124. Azizi Samir, M.A.S.; Alloin, F.; Sanchez, J.Y.; Dufresne, A. POE-based nanocomposite polymer electrolytes reinforced with cellulose whiskers. Electrochim. Acta 2005, 50, 3897-3903. [CrossRef]

125. Azizi Samir, M.A.S.; Alloin, F.; Dufresne, A. High performance nanocomposite polymer electrolytes. Compos. Interfaces 2006, 13, 545-559. [CrossRef]

126. Selvakumar, M.; Bhat, D.K. $\mathrm{LiClO}_{4}$ doped cellulose acetate as biodegradable polymer electrolyte for supercapacitors. J. Appl. Polym. Sci. 2008, 110, 594-602. [CrossRef]

127. Lin, S.-Y.; Wang, C.-M.; Hsieh, P.-T.; Chen, Y.-C.; Liu, C.-C.; Shih, S.-C. A novel gel polymer electrolyte based on lithium salt with an ethyl cellulose. Colloid Polym. Sci. 2009, 287, 1355-1358. [CrossRef]

128. Ren, Z.; Liu, Y.; Sun, K.; Zhou, X.; Zhang, N. A microporous gel electrolyte based on poly(vinylidene fluoride-co-hexafluoropropylene)/fully cyanoethylated cellulose derivative blend for lithium-ion battery. Electrochim. Acta 2009, 54, 1888-1892. [CrossRef]

129. Johari, N.A.; Kudin, T.I.T.; Ali, A.M.M.; Winie, T.; Yahya, M.Z.A. Studies on cellulose acetate-based gel polymer electrolytes for proton batteries. Mater. Res. Innov. 2009, 13, 232-234. [CrossRef]

130. Lee, J.M.; Nguyen, D.Q.; Lee, S.B.; Kim, H.; Ahn, B.S.; Lee, H.; Kim, H.S. Cellulose triacetate-based polymer gel electrolytes. J. Appl. Polym. Sci. 2010, 115, 32-36. [CrossRef]

131. Li, P.; Zhang, Y.; Fa, W.; Zhang, Y.; Huang, B. Synthesis of a grafted cellulose gel electrolyte in an ionic liquid ([Bmim]I) for dye-sensitized solar cells. Carbohydr. Polym. 2011, 86, 1216-1220. [CrossRef]

132. Ali, R.M.; Harun, N.I.; Ali, A.M.M.; Yahya, M.Z.A. Conductivity studies on plasticised cellulose acetate-ammonium iodide based polymer electrolytes. Mater. Res. Innov. 2011, 15, 39-42. [CrossRef]

133. Johari, N.A.; Kudin, T.I.T.; Ali, A.M.M.; Yahya, M.Z.A. Effects of $\mathrm{TiO}_{2}$ on conductivity performance of cellulose acetate based polymer gel electrolytes for proton batteries. Mater. Res. Innov. 2011, 15, 229-231. [CrossRef] 
134. Harun, N.I.; Ali, R.M.; Ali, A.M.M.; Yahya, M.Z.A. Conductivity studies on cellulose acetate-tetrafluoroborate based polymer electrolytes. Mater. Res. Innov. 2011, 15, 39-42. [CrossRef]

135. Harun, N.I.; Ali, R.M.; Ali, A.M.M.; Yahya, M.Z.A. Dielectric behaviour of cellulose acetate-based polymer electrolytes. Ionics 2011, 18, 599-606. [CrossRef]

136. Harun, N.I.; Md Ali, R.; Ali, A.M.M.; Yahya, M.Z.A. Effects of ammonium tetrafluoroborate on conductivity and thermal studies on cellulose acetate based polymer electrolytes. Adv. Mater. Res. 2013, 667, 150-154. [CrossRef]

137. Samsudin, A.S.; Khairul, W.M.; Isa, M.I.N. Characterization on the potential of carboxy methylcellulose for application as proton conducting biopolymer electrolytes. J. Non. Cryst. Solids 2012, 358, 1104-1112. [CrossRef]

138. Asghar, A.; Abdul Samad, Y.; Singh Lalia, B.; Hashaikeh, R. PEG based quasi-solid polymer electrolyte: Mechanically supported by networked cellulose. J. Memb. Sci. 2012, 421-422, 85-90. [CrossRef]

139. Huang, X.; Liu, Y.; Deng, J.; Yi, B.; Yu, X.; Shen, P.; Tan, S. A novel polymer gel electrolyte based on cyanoethylated cellulose for dye-sensitized solar cells. Electrochim. Acta 2012, 80, 219-226. [CrossRef]

140. Ramesh, S.; Shanti, R.; Morris, E. Discussion on the influence of DES content in CA-based polymer electrolytes. J. Mater. Sci. 2012, 47, 1787-1793. [CrossRef]

141. Ramesh, S.; Shanti, R.; Morris, E. Characterization of conducting cellulose acetate based polymer electrolytes doped with 'green' ionic mixture. Carbohydr. Polym. 2013, 91, 14-21. [CrossRef] [PubMed]

142. Ramesh, S.; Shanti, R.; Morris, E. Plasticizing effect of 1-allyl-3-methylimidazolium chloride in cellulose acetate based polymer electrolytes. Carbohydr. Polym. 2012, 87, 2624-2629. [CrossRef]

143. Sahli, N.B.; Bin Ali, A.M. Effect of lithium triflate salt concentration in methyl cellulose-based solid polymer electrolytes. In Proceedings of the 2012 IEEE Colloquium on Humanities, Science and Engineering (CHUSER), Kota Kinabalu, Malaysia, 3-4 December 2012; pp. 739-742.

144. Shuhaimi, N.E.A.; Teo, L.P.; Woo, H.J.; Majid, S.R.; Arof, A.K. Electrical double-layer capacitors with plasticized polymer electrolyte based on methyl cellulose. Polym. Bull. 2012, 69, 807-826. [CrossRef]

145. Mustafa, M.F.; Ridwan, N.I.M.; Hatta, F.F.; Yahya, M.Z.A. Effect of dimethyl carbonate plasticizer on ionic cnductivity of methyl cellulose-based polymer electrolyte. Malays. J. Anal. Sci. 2012, 16, 283-289.

146. Liu, J.; Li, W.; Zuo, X.; Liu, S.; Li, Z. Polyethylene-supported polyvinylidene fluoride-cellulose acetate butyrate blended polymer electrolyte for lithium ion battery. J. Power Sources 2013, 226, 101-106. [CrossRef]

147. Bella, F.; Nair, J.R.; Gerbaldi, C. Towards green, efficient and durable quasi-solid dye-sensitized solar cells integrated with a cellulose-based gel-polymer electrolyte optimized by a chemometric DoE approach. RSC Adv. 2013, 3, 15993-16001. [CrossRef]

148. Abidin, S.Z.Z.; Ali, A.M.M.; Hassan, O.H.; Yahya, M.Z.A. Electrochemical studies on cellulose acetate-LiBOB polymer gel electrolytes. Int. J. Electrochem. Sci. 2013, 8, 7320-7326.

149. Samad, Y.A.; Asghar, A.; Lalia, B.S.; Hashaikeh, R. Networked cellulose entrapped and reinforced PEO-based solid polymer electrolyte for moderate temperature applications. J. Appl. Polym. Sci. 2013, 129, 2998-3006. [CrossRef]

150. Ran, Y.; Yin, Z.; Ding, Z.; Guo, H.; Yang, J. A polymer electrolyte based on poly(vinylidene fluoride-hexafluoropylene)/hydroxypropyl methyl cellulose blending for lithium-ion battery. Ionics 2013, 19, 757-762. [CrossRef]

151. Ramesh, S.; Shanti, R.; Morris, E. Employment of [Amim] Cl in the effort to upgrade the properties of cellulose acetate based polymer electrolytes. Cellulose 2013, 20, 1377-1389. [CrossRef]

152. Kiristi, M.; Bozduman, F.; Gulec, A.; Teke, E.; Oksuz, L.; Oksuz, A.U.; Deligoz, H. Complementary all solid state electrochromic devices using carboxymethyl cellulose based electrolytes. J. Macromol. Sci. Part A 2014, 51, 481-487. [CrossRef]

153. Arof, A.K.; Amirudin, S.; Yusof, S.Z.; Noor, I.M. A method based on impedance spectroscopy to determine transport properties of polymer electrolytes. Phys. Chem. Chem. Phys. 2014, 16, 1856-1867. [CrossRef]

154. Chiappone, A.; Bella, F.; Nair, J.R.; Meligrana, G.; Bongiovanni, R.; Gerbaldi, C. Structure-performance correlation of nanocellulose-based polymer electrolytes for efficient quasi-solid DSSCs. ChemElectroChem 2014, 1, 1350-1358. [CrossRef]

155. Xiao, S.Y.; Wang, F.X.; Chang, Z.; Wu, Y.P. An environmentally friendly and economic membrane based on cellulose as a gel polymer electrolyte for lithium ion batteries. J. Power Sources 2014, 270, 53-58. [CrossRef] 
156. Ng, W.F.; Chai, M.N.; Isa, M.I.N. Proton conducting carboxy methyl cellulose solid polymer electrolytes doped with citric acid. Adv. Mater. Res. 2014, 895, 130-133. [CrossRef]

157. Jafirin, S.; Ahmad, I.; Ahmad, A. Composite polymer electrolyte based on MG-49 and carboxymethyl cellulose from kenaf. AIP Conf. Proc. 2014, 1571, 822-828.

158. Abiddin, J.F.Z.; Ahmad, A.H. Fourier transform infrared spectroscopy and electrical characterization of methylcellulose based solid polymer electrolyte doped with sodium iodide. Jurnal Teknologi 2015, 3, 41-45.

159. Abiddin, J.F.Z.; Ahmad, A.H. Conductivity study and fourier transform infrared (FTIR) characterization of methyl cellulose solid polymer electrolyte with sodium iodide conducting ion. AIP Conf. Proc. 2015, 1674, $1-6$.

160. Li, M.; Wang, X.; Wang, Y.; Chen, B.; Wu, Y.; Holze, R. A gel polymer electrolyte based on composite of nonwoven fabric and methyl cellulose with good performance for lithium ion batteries. RSC Adv. 2015, 5, 52382-52387. [CrossRef]

161. Leng, L.; Zeng, X.; Chen, P.; Shu, T.; Song, H.; Fu, Z.; Wang, H.; Liao, S. A novel stability-enhanced lithium-oxygen battery with cellulose-based composite polymer gel as the electrolyte. Electrochim. Acta 2015, 176, 1108-1115. [CrossRef]

162. Colò, F.; Bella, F.; Nair, J.R.; Destro, M.; Gerbaldi, C. Cellulose-based novel hybrid polymer electrolytes for green and efficient Na-ion batteries. Electrochim. Acta 2015, 174, 185-190. [CrossRef]

163. Razalli, S.M.M.; Sheikh Mohd Saaid, S.I.Y.; Marwan Ali, A.M.; Hassan, O.H.; Yahya, M.Z.A. Cellulose acetate-lithium bis(trifluoromethanesulfonyl)imide solid polymer electrolyte: ATR-FTIR and ionic conductivity behavior. Funct. Mater. Lett. 2015, 8, 1-4.

164. Sudhakar, Y.N.; Selvakumar, M.; Bhat, D.K. Preparation and characterization of phosphoric acid-doped hydroxyethyl cellulose electrolyte for use in supercapacitor. Mater. Renew. Sustain. Energy 2015, 4, 1-9. [CrossRef]

165. Zhu, Y.S.; Xiao, S.Y.; Li, M.X.; Chang, Z.; Wang, F.X.; Gao, J.; Wu, Y.P. Natural macromolecule based carboxymethyl cellulose as a gel polymer electrolyte with adjustable porosity for lithium ion batteries. J. Power Sources 2015, 288, 368-375. [CrossRef]

166. Ledwon, P.; Andrade, J.R.; Lapkowski, M.; Pawlicka, A. Hydroxypropyl cellulose-based gel electrolyte for electrochromic devices. Electrochim. Acta 2015, 159, 227-233. [CrossRef]

167. Samsi, N.S.; Zakaria, R.; Hassan, O.H.; Yahya, M.Z.A.; Ali, A.M.M. Optical behavior of $\mathrm{NH}_{4} \mathrm{I}$ doped cellulose acetate membrane for dye sensitized solar cell. Malays. J. Anal. Sci. 2015, 19, 760-765.

168. Rani, M.S.A.; Dzulkurnain, N.A.; Ahmad, A.; Mohamed, N.S. Conductivity and dielectric behavior studies of carboxymethyl cellulose from kenaf bast fiber incorporated with ammonium acetate-BMATFSI biopolymer electrolytes. Int. J. Polym. Anal. Charact. 2015, 20, 250-260. [CrossRef]

169. Li, M.X.; Wang, X.W.; Yang, Y.Q.; Chang, Z.; Wu, Y.P.; Holze, R. A dense cellulose-based membrane as a renewable host for gel polymer electrolyte of lithium ion batteries. J. Memb. Sci. 2015, 476, 112-118. [CrossRef]

170. Ahmad, N.H.B.; Isa, M.I.N. Proton conducting solid polymer electrolytes based carboxymethyl cellulose doped ammonium chloride: Ionic conductivity and transport studies. Int. J. Plast. Technol. 2015, 19, 47-55. [CrossRef]

171. Sohaimy, M.I.H.; Isa, M.I.N. Effect of ammonium carbonate salt concentration on structural and ionic conductivity of cellulose based solid polymer electrolytes. Fibers Polym. 2015, 16, 1031-1034. [CrossRef]

172. Ramlli, M.A.; Isa, M.I.N. Structural and ionic transport properties of protonic conducting solid biopolymer electrolytes based on carboxymethyl cellulose doped with ammonium fluoride. J. Phys. Chem. B 2016, 120, 11567-11573. [CrossRef] [PubMed]

173. Chong, M.Y.; Liew, C.W.; Numan, A.; Yugal, K.; Ramesh, K.; Ng, H.M.; Chong, T.V.; Ramesh, S. Effects of ionic liquid on the hydroxylpropylmethyl cellulose (HPMC) solid polymer electrolyte. Ionics 2016, 22, 2421-2430. [CrossRef]

174. Chai, M.N.; Isa, M.I.N. Novel proton conducting solid bio-polymer electrolytes based on carboxymethyl cellulose doped with oleic acid and plasticized with glycerol. Sci. Rep. 2016, 6, 1-7. [CrossRef]

175. Ngamaroonchote, A.; Chotsuwan, C. Performance and reliability of cellulose acetate-based gel electrolyte for electrochromic devices. J. Appl. Electrochem. 2016, 46, 575-582. [CrossRef] 
176. Samsi, N.S.; Zakaria, R.; Hassan, O.H.; Azhan Yahya, M.Z.; Ali, A.M.M. X-ray diffraction and infrared studies on plasticized cellulose acetate complexed with ammonium iodide for solid polymer electrolyte. Mater. Sci. Forum 2016, 846, 523-527. [CrossRef]

177. Zhao, M.; Zuo, X.; Wang, C.; Xiao, X.; Liu, J.; Nan, J. Preparation and performance of the polyethylene-supported polyvinylidene fluoride/cellulose acetate butyrate/nano- $\mathrm{SiO}_{2}$ particles blended gel polymer electrolyte. Ionics 2016, 22, 2123-2132. [CrossRef]

178. Monisha, S.; Mathavan, T.; Selvasekarapandian, S.; Benial, A.M.F.; Latha, M.P. Preparation and characterization of cellulose acetate and lithium nitrate for advanced electrochemical devices. Ionics 2016, 23, 1-10. [CrossRef]

179. Monisha, S.; Selvasekarapandian, S.; Mathavan, T.; Milton Franklin Benial, A.; Manoharan, S.; Karthikeyan, S. Preparation and characterization of biopolymer electrolyte based on cellulose acetate for potential applications in energy storage devices. J. Mater. Sci. Mater. Electron. 2016, 27, 9314-9324. [CrossRef]

180. Razalli, S.M.M.; Saaid, S.I.Y.S.M.; Kudin, T.I.T.; Yahya, M.Z.A.; Hassan, O.H.; Ali, A.M.M. Electrochemical properties of glyme based plasticizer on gel polymer electrolytes doped with lithium bis(trifluoromethanesulfonyl)imide. Mater. Sci. Forum 2016, 846, 534-538. [CrossRef]

181. Song, A.; Huang, Y.; Zhong, X.; Cao, H.; Liu, B.; Lin, H.; Wang, M.; Li, X. Gel polymer electrolyte with high performances based on pure natural polymer matrix of potato starch composite lignocellulose. Electrochim. Acta 2017, 245, 981-992. [CrossRef]

182. Song, A.; Huang, Y.; Liu, B.; Cao, H.; Zhong, X.; Lin, Y.; Wang, M.; Li, X.; Zhong, W. Gel polymer electrolyte based on polyethylene glycol composite lignocellulose matrix with higher comprehensive performances. Electrochim. Acta 2017, 247, 505-515. [CrossRef]

183. Gaur, S.S.; Dhar, P.; Sonowal, A.; Sharma, A.; Kumar, A.; Katiyar, V. Thermo-mechanically stable sustainable polymer based solid electrolyte membranes for direct methanol fuel cell applications. J. Memb. Sci. 2017, 526, 348-354. [CrossRef]

184. Gupta, S.; Varshney, P.K. Effect of plasticizer concentration on structural and electrical properties of hydroxyethyl cellulose (HEC)-based polymer electrolyte. Ionics 2017, 23, 1613-1617. [CrossRef]

185. Monisha, S.; Mathavan, T.; Selvasekarapandian, S.; Benial, A.M.F.; Aristatil, G.; Mani, N.; Premalatha, M.; Pandi, D.V. Investigation of bio polymer electrolyte based on cellulose acetate-ammonium nitrate for potential use in electrochemical devices. Carbohydr. Polym. 2017, 157, 38-47. [CrossRef]

186. Sohaimy, M.I.H.; Isa, M.I.N. Ionic conductivity and conduction mechanism studies on cellulose based solid polymer electrolytes doped with ammonium carbonate. Polym. Bull. 2017, 74, 1371-1386. [CrossRef]

187. Mohamed, N.S.; Subban, R.H.Y.; Arof, A.K. Polymer batteries fabricated from lithium complexed acetylated chitosan. J. Power Sources 1995, 56, 153-156. [CrossRef]

188. Subban, R.H.Y.; Arof, A.K. Sodium iodide added chitosan electrolyte film for polymer batteries. Phys. Scr. 1996, 53, 382. [CrossRef]

189. Subban, R.H.Y.; Arof, A.K.; Radhakrishna, S. Polymer batteries with chitosan electrolyte mixed with sodium perchlorate. Mater. Sci. Eng. B 1996, 38, 156-160. [CrossRef]

190. Velazquez-Morales, P.; Le Nest, J.-F.; Gandini, A. Polymer electrolytes derived from chitosan/polyether networks. Electrochim. Acta 1998, 43, 1275-1279. [CrossRef]

191. Osman, Z.; Arof, A.K. FTIR studies of chitosan acetate based polymer electrolytes. Electrochim. Acta 2003, 48, 993-999. [CrossRef]

192. De Vasconcelos, C.L.; Rocha, A.N.L.; Pereira, M.R.; Fonseca, J.L.C. Electrolyte diffusion in a chitosan membrane. Polym. Int. 2001, 50, 309-312. [CrossRef]

193. Kamarulzaman, N.; Osman, Z.; Muhamad, M.R.; Ibrahim, Z.A. Performance characteristics of $\mathrm{LiMn}_{2} \mathrm{O}_{4} /$ polymer/carbon electrochemical cells. J. Power Sources 2001, 97-98, 722-725. [CrossRef]

194. Yahya, M.Z.A.; Arof, A.K. Characteristics of chitosan-lithium acetate-palmitic acid complexes. J. New Mater. Electrochem. Syst. 2002, 5, 123-128.

195. Yahya, M.Z.A.; Arof, A.K. Studies on lithium acetate doped chitosan conducting polymer system. Eur. Polym. J. 2002, 38, 1191-1197. [CrossRef]

196. Yahya, M.Z.A.; Arof, A.K. Conductivity and X-ray photoelectron studies on lithium acetate doped chitosan films. Carbohydr. Polym. 2004, 55, 95-100. [CrossRef] 
197. Ali, A.M.M.; Yahya, M.Z.A.; Mustaffa, M.; Ahmad, A.H.; Subban, R.H.Y.; Harun, M.K.; Mohamad, A.A. Electrical properties of plasticized chitosan-lithium imide with oleic acid-based polymer electrolytes for lithium rechargeable batteries. Ionics 2005, 11, 460-463. [CrossRef]

198. Wan, Y.; Creber, K.A.M.; Peppley, B.; Tam Bui, V.; Halliop, E. New solid polymer electrolyte membranes for alkaline fuel cells. Polym. Int. 2005, 54, 5-10. [CrossRef]

199. Majid, S.R.; Idris, N.H.; Hassan, M.F.; Winie, T.; Khiar, A.S.A.; Arof, A.K. Transport studies on filler-doped chitosan based polymer electrolyte. Ionics 2005, 11, 451-455. [CrossRef]

200. Winie, T.; Majid, S.R.; Hassan, M.F.; Arof, A.K. Characterization of plasticized hexanoyl chitosan-based polymer electrolytes and application in $\mathrm{LiCoO}_{2} / \mathrm{MCMB}$ cells. Mater. Sci. Forum 2006, 517, 85-88. [CrossRef]

201. Winie, T.; Arof, A.K. FT-IR studies on interactions among components in hexanoyl chitosan-based polymer electrolytes. Spectrochim. Acta A Mol. Biomol. Spectrosc. 2006, 63, 677-684. [CrossRef] [PubMed]

202. Khiar, A.S.A.; Majid, S.R.; Idris, N.H.; Hassan, M.F.; Puteh, R.; Arof, A.K. Ionic hopping transport in chitosan-based polymer electrolytes. Mater. Sci. Forum 2006, 517, 237-241. [CrossRef]

203. Yahya, M.Z.A.; Ali, A.M.M.; Mohammat, M.F.; Hanafiah, M.A.K.M.; Mustaffa, M.; Ibrahim, S.C.; Darus, Z.M.; Harun, M.K. Ionic conduction model in salted chitosan membranes plasticized with fatty acid. J. Appl. Sci. 2006, 6, 1287-1291.

204. Winie, T.; Majid, S.R.; Khiar, A.S.A.; Arof, A.K. Ionic conductivity of chitosan membranes and application for electrochemical devices. Polym. Adv. Technol. 2006, 17, 523-527. [CrossRef]

205. Wan, Y.; Peppley, B.; Creber, K.A.M.; Bui, V.T.; Halliop, E. Preliminary evaluation of an alkaline chitosan-based membrane fuel cell. J. Power Sources 2006, 162, 105-113. [CrossRef]

206. Ng, L.S.; Mohamad, A.A. Protonic battery based on a plasticized chitosan- $\mathrm{NH}_{4} \mathrm{NO}_{3}$ solid polymer electrolyte. J. Power Sources 2006, 163, 382-385. [CrossRef]

207. Winie, T.; Arof, A.K. Transport properties of hexanoyl chitosan-based gel electrolyte. Ionics 2006, 12, 149-152. [CrossRef]

208. Idris, N.H.; Senin, H.B.; Arof, A.K. Dielectric spectra of LiTFSI-doped chitosan/PEO blends. Ionics 2007, 13, 213-217. [CrossRef]

209. Majid, S.R.; Arof, A.K. Mobility and density of ions in chitosan-orthophosphoric acid-ammonium nitrate electrolytes. Phys. Status Solidi Appl. Mater. Sci. 2007, 204, 2396-2401. [CrossRef]

210. Mohamad, S.A.; Ali, M.H.; Yahya, R.; Ibrahim, Z.A.; Arof, A.K. Photovoltaic activity in a ZnSe/PEO-chitosan blend electrolyte junction. Ionics 2007, 13, 235-240. [CrossRef]

211. Fuentes, S.; Retuert, P.J.; González, G. Lithium ion conductivity of molecularly compatibilized chitosan-poly(aminopropyltriethoxysilane)-poly(ethylene oxide) nanocomposites. Electrochim. Acta 2007, 53, 1417-1421. [CrossRef]

212. Pawlicka, A.; Danczuk, M.; Wieczorek, W.; Zygadło-Monikowska, E. Influence of plasticizer type on the properties of polymer electrolytes based on chitosan. J. Phys. Chem. A 2008, 112, 8888-8895. [CrossRef] [PubMed]

213. Göktepe, F.; Çelik, S.U.; Bozkurt, A. Preparation and the proton conductivity of chitosan/poly(vinyl phosphonic acid) complex polymer electrolytes. J. Non-Cryst. Solids 2008, 354, 3637-3642.

214. Ng, L.S.; Mohamad, A.A. Effect of temperature on the performance of proton batteries based on chitosan- $\mathrm{NH}_{4} \mathrm{NO}_{3}$-EC membrane. J. Membr. Sci. 2008, 325, 653-657. [CrossRef]

215. Idris, N.K.; Aziz, N.A.N.; Zambri, M.S.M.; Zakaria, N.A.; Isa, M.I.N. Ionic conductivity studies of chitosan-based polymer electrolytes doped with adipic acid. Ionics 2009, 15, 643-646. [CrossRef]

216. Majid, S.R.; Arof, A.K. Conductivity studies and performance of chitosan based polymer electrolytes in $\mathrm{H}_{2}$ /air fuel cell. Polym. Adv. Technol. 2009, 20, 524-528. [CrossRef]

217. Kadir, M.F.Z.A.; Teo, L.P.; Majid, S.R.; Arof, A.K. Conductivity studies on plasticised PEO/chitosan proton conducting polymer electrolyte. Mater. Res. Innov. 2009, 13, 259-262. [CrossRef]

218. Du, J.F.; Bai, Y.; Pan, D.A.; Chu, W.Y.; Qiao, L.J. Characteristics of proton conducting polymer electrolyte based on chitosan acetate complexed with $\mathrm{CH}_{3} \mathrm{COONH}_{4}$. J. Polym. Sci. Part B Polym. Phys. 2009, 47, 549-554. [CrossRef]

219. Muhammad, F.H.; Subban, R.H.Y.; Majid, S.R.; Winie, T.; Arof, A.K. Characterisation of $\mathrm{Al}_{2} \mathrm{O}_{3}$ doped hexanoyl chitosan-LiCF $3 \mathrm{SO}_{3}-\mathrm{EC}$ polymer electrolytes. Mater. Res. Innov. 2009, 13, 249-251. [CrossRef]

220. Muhammad, F.H.; Subban, R.H.Y.; Winie, T. Electrical studies on hexanoyl chitosan-based nanocomposite polymer electrolytes. AIP Conf. Proc. 2009, 1136, 61-65. 
221. Rosli, N.H.A.; Muhammad, F.H.; Subban, R.H.Y.; Winie, T. Structural and electrical studies of hexanoyl chitosan based electrolyte system. Mater. Res. Innov. 2011, 15, 1-4. [CrossRef]

222. Winie, T.; Ramesh, S.; Arof, A.K. Studies on the structure and transport properties of hexanoyl chitosan-based polymer electrolytes. Phys. B Condens. Matter 2009, 404, 4308-4311. [CrossRef]

223. Kumar, M.S.; Bhat, D.K. $\mathrm{LiClO}_{4}$-doped plasticized chitosan as biodegradable polymer gel electrolyte for supercapacitors. J. Appl. Polym. Sci. 2009, 114, 2445-2454. [CrossRef]

224. Arof, A.K.; Buraidah, M.H.; Teo, L.P.; Majid, S.R.; Yahya, R.; Taha, R.M. Characterizations of chitosan-based polymer electrolyte photovoltaic cells. Int. J. Photoenergy 2010, 2010, 1-7.

225. Buraidah, M.H.; Teo, L.P.; Majid, S.R.; Arof, A.K. Characteristics of $\mathrm{TiO}_{2} /$ solid electrolyte junction solar cells with redox couple. Opt. Mater. 2010, 32, 723-728. [CrossRef]

226. Du, J.F.; Bai, Y.; Chu, W.Y.; Qiao, L.J. Synthesis and performance of proton conducting chitosan/ $\mathrm{NH}_{4} \mathrm{Cl}$ electrolyte. J. Polym. Sci. Part B Polym. Phys. 2010, 48, 260-266. [CrossRef]

227. Aziz, S.B.; Abidin, Z.H.Z.; Arof, A.K. Influence of silver ion reduction on electrical modulus parameters of solid polymer electrolyte based on chitosansilver triflate electrolyte membrane. Express Polym. Lett. 2010, 4, 300-310. [CrossRef]

228. Singh, P.K.; Bhattacharya, B.; Nagarale, R.K.; Kim, K.-W.; Rhee, H.-W. Synthesis, characterization and application of biopolymer-ionic liquid composite membranes. Synth. Met. 2010, 160, 139-142. [CrossRef]

229. Kadir, M.F.Z.; Majid, S.R.; Arof, A.K. Plasticized chitosan-PVA blend polymer electrolyte based proton battery. Electrochim. Acta 2010, 55, 1475-1482. [CrossRef]

230. Jaafar, N.K.; Lepit, A.; Aini, N.A.; Saat, A.; Ali, A.M.M.; Yahya, M.Z.A. Effects of lithium salt on chitosan-g-PMMA based polymer electrolytes. Mater. Res. Innov. 2011, 15, 202-205. [CrossRef]

231. Buraidah, M.H.; Teo, L.P.; Yusuf, S.N.F.; Noor, M.M.; Kufian, M.Z.; Careem, M.A.; Majid, S.R.; Taha, R.M.; Arof, A.K. TiO 2 chitosan- $\mathrm{NH}_{4} \mathrm{I}\left(+\mathrm{I}_{2}\right)$-BMII-based dye-sensitized solar cells with anthocyanin dyes extracted from black rice and red cabbage. Int. J. Photoenergy 2011, 2011, 1-11. [CrossRef]

232. Aziz, N.A.; Majid, S.R.; Yahya, R.; Arof, A.K. Conductivity, structure, thermal properties of chitosan-based polymer electrolytes with nanofillers. Polym. Adv. Technol. 2011, 22, 1345-1348. [CrossRef]

233. Winie, T.; Han, C.C.; Subban, R.H.Y. Ac conductivity and dielectric properties of hexanoyl chitosan- $\mathrm{LiClO}_{4}-\mathrm{TiO}_{2}$ composite polymer electrolytes. Adv. Mater. Res. 2011, 335-336, 873-880. [CrossRef]

234. Kadir, M.F.Z.; Aspanut, Z.; Yahya, R.; Arof, A.K. Chitosan-PEO proton conducting polymer electrolyte membrane doped with $\mathrm{NH}_{4} \mathrm{NO}_{3}$. Mater. Res. Innov. 2011, 15, 164-167. [CrossRef]

235. Kadir, M.F.Z.; Arof, A.K. Application of PVA-chitosan blend polymer electrolyte membrane in electrical double layer capacitor. Mater. Res. Innov. 2011, 15, 217-220. [CrossRef]

236. Navaratnam, S.; Ramesh, K.; Basirun, W.J. Investigation of ion conducting behaviour of composite chitosan based polymer electrolytes. Mater. Res. Innov. 2011, 152, 184-186. [CrossRef]

237. Buraidah, M.H.; Arof, A.K. Characterization of chitosan/PVA blended electrolyte doped with $\mathrm{NH}_{4} \mathrm{I}$. J. Non. Cryst. Solids 2011, 357, 3261-3266. [CrossRef]

238. Mattos, R.I.; Raphael, E.; Majid, S.R.; Arof, A.K.; Pawlicka, A. Enhancement of electrical conductivity in plasticized chitosan based membranes. Mol. Cryst. Liq. Cryst. 2012, 554, 150-159. [CrossRef]

239. Aziz, N.A.; Majid, S.R.; Arof, A.K. Synthesis and characterizations of phthaloyl chitosan-based polymer electrolytes. J. Non. Cryst. Solids 2012, 358, 1581-1590. [CrossRef]

240. Xiong, Y.; Wang, H.; Wu, C.; Wang, R. Preparation and characterization of conductive chitosan-ionic liquid composite membranes. Polym. Adv. Technol. 2012, 23, 1429-1434. [CrossRef]

241. Winie, T.; Muhammad, F.H.; Rosli, N.H.A. Effect of anion size on the conductivity behaviour of hexanoyl chitosan-based polymer electrolytes. Adv. Mater. Res. 2012, 545, 317-320. [CrossRef]

242. Shukur, M.F.; Kadir, M.F.Z.; Ahmad, Z.; Ithnin, R. Dielectric studies of proton conducting polymer electrolyte based on chitosan/PEO blend doped with $\mathrm{NH}_{4} \mathrm{NO}_{3}$. Adv. Mater. Res. 2012, 488-489, 583-587. [CrossRef]

243. Shukur, M.F.; Kadir, M.F.Z.; Ahmad, Z.; Ithnin, R. Transport properties of chitosan/PEO blend based proton conducting polymer electrolyte. Adv. Mater. Res. 2012, 488-489, 114-117. [CrossRef]

244. Karuppasamy, K.; Thanikaikarasan, S.; Antony, R.; Balakumar, S.; Shajan, X.S. Effect of nanochitosan on electrochemical, interfacial and thermal properties of composite solid polymer electrolytes. Ionics 2012, 18, 737-745. [CrossRef]

245. Alaei, I.; Al-Bat'hi, S.A.M. $\mathrm{TiO}_{2} /$ solid state polymer junction for photovoltaic application. Adv. Mater. Res. 2012, 576, 623-625. [CrossRef] 
246. Mobarak, N.N.; Ramli, N.; Ahmad, A.; Abdullah, M.P. Development of carboxymethyl chitosan based green polymer electrolyte. J. Biobased Mater. Bioenergy 2013, 7, 267-268. [CrossRef]

247. Mobarak, N.N.; Ahmad, A.; Abdullah, M.P.; Ramli, N.; Rahman, M.Y.A. Conductivity enhancement via chemical modification of chitosan based green polymer electrolyte. Electrochim. Acta 2013, 92, 161-167. [CrossRef]

248. Sudhakar, Y.N.; Selvakumar, M.; Bhat, D.K. $\mathrm{LiClO}_{4}$-doped plasticized chitosan and poly(ethylene glycol) blend as biodegradable polymer electrolyte for supercapacitors. Ionics 2013, 19, 277-285. [CrossRef]

249. Winie, T.; Hanif, N.S.M.; Rosli, N.H.A.; Subban, R.H.Y. Ac conductivity study of hexanoyl chitosan- $\mathrm{LiCF}_{3} \mathrm{SO}_{3}-\mathrm{EC}-\mathrm{Al}_{2} \mathrm{O}_{3}$ nanocomposite polymer electrolytes. Adv. Mater. Res. 2013, 667, 93-98. [CrossRef]

250. Shukur, M.F.; Azmi, M.S.; Zawawi, S.M.M.; Majid, N.A.; Illias, H.A.; Kadir, M.F.Z. Conductivity studies of biopolymer electrolytes based on chitosan incorporated with $\mathrm{NH}_{4}$ Br. Phys. Scr. 2013, 2013, 1-6. [CrossRef]

251. Hassan, F.; Woo, H.J.; Aziz, N.A.; Kufian, M.Z.; Majid, S.R. Synthesis of $\mathrm{Al}_{2} \mathrm{TiO}_{5}$ and its effect on the properties of chitosan- $\mathrm{NH}_{4} \mathrm{SCN}$ polymer electrolytes. Ionics 2013, 19, 483-489. [CrossRef]

252. Hamdan, K.Z.; Khiar, A.S.A. Conductivity and dielectric studies of methylcellulose/chitosan- $\mathrm{NH}_{4} \mathrm{CF}_{3} \mathrm{SO}_{3}$ polymer electrolyte. Key Eng. Mater. 2014, 594-595, 818-822.

253. Mobarak, N.N.; Ramli, N.; Abdullah, M.P.; Ahmad, A. Spectroscopic studies of carboxymethyl chitosan-ammonium triflate $\left(\mathrm{NH}_{4} \mathrm{CF}_{3} \mathrm{SO}_{3}\right)$ based solid polymer electrolytes. AIP Conf. Proc. 2014, 1571, 843-849.

254. Leones, R.; Sentanin, F.; Esperança, J.M.S.S.; Pawlicka, A.; De Zea Bermudez, V.; Silva, M.M. Chitosan and ionic liquid based solid polymer electrolytes: The anion alkyl chain length effect. ECS Trans. 2014, 61, 51-59. [CrossRef]

255. Rosli, N.H.A.; Muhammad, F.H.; Han, C.; Winie, T. Effect of filler type on the electrical properties of hexanoyl chitosan- based polymer electrolytes. Adv. Mater. Res. 2014, 832, 224-227. [CrossRef]

256. Jaafar, N.K.; Lepit, A.; Aini, N.A.; Ali, A.M.M.; Saat, A.; Yahya, M.Z.A. Structural and electrical properties of plasticized radiation induced chitosan grafted poly(methylmethacrylate) polymer electrolytes. Int. J. Electrochem. Sci. 2014, 9, 821-829.

257. Taib, N.U.; Idris, N.H. Plastic crystal-solid biopolymer electrolytes for rechargeable lithium batteries. J. Membr. Sci. 2014, 468, 149-154. [CrossRef]

258. Hafiza, M.N.; Bashirah, A.N.A.; Bakar, N.Y.; Isa, M.I.N. Electrical properties of carboxyl methylcellulose/chitosan dual-blend green polymer doped with ammonium bromide. Int. J. Polym. Anal. Charact. 2014, 19, 151-158. [CrossRef]

259. Winie, T.; Jamal, A.; Hanif, N.S.M.; Shahril, N.S.M. Hexanoyl chitosan-polystyrene blend based composite polymer electrolyte with surface treated $\mathrm{TiO}_{2}$ fillers. Key Eng. Mater. 2014, 594-595, 656-660.

260. Winie, T.; Hanif, N.S.M.; Chan, C.H.; Arof, A.K. Effect of the surface treatment of the $\mathrm{TiO}_{2}$ fillers on the properties of hexanoyl chitosan/polystyrene blend-based composite polymer electrolytes. Ionics 2014, 20, 347-352. [CrossRef]

261. Winie, T.; Mohd Shahril, N.S. Conductivity enhancement by controlled percolation of inorganic salt in multiphase hexanoyl chitosan/polystyrene polymer blends. Front. Mater. Sci. 2015, 9, 132-140. [CrossRef]

262. Yusof, Y.M.; Illias, H.A.; Kadir, M.F.Z. Incorporation of $\mathrm{NH}_{4} \mathrm{Br}$ in PVA-chitosan blend-based polymer electrolyte and its effect on the conductivity and other electrical properties. Ionics 2014, 20, 1235-1245. [CrossRef]

263. Begum, S.N.S.; Pandian, R.; Aswal, V.K.; Ramasamy, R.P. Chitosan-gold-lithium nanocomposites as solid polymer electrolyte. J. Nanosci. Nanotechnol. 2014, 14, 5761-5773. [CrossRef]

264. Fauzi, I.; Arcana, I.M. Solid polymer electrolyte from phosphorylated chitosan. AIP Conf. Proc. 2014, 1589, 154-158.

265. Fadzallah, I.A.; Majid, S.R.; Careem, M.A.; Arof, A.K. Relaxation process in chitosan-oxalic acid solid polymer electrolytes. Ionics 2014, 20,969-975. [CrossRef]

266. Fadzallah, I.A.; Majid, S.R.; Careem, M.A.; Arof, A.K. A study on ionic interactions in chitosan-oxalic acid polymer electrolyte membranes. J. Memb. Sci. 2014, 463, 65-72. [CrossRef]

267. Fauzi, I.; Arcana, I.M.; Wahyuningrum, D. Synthesis and characterization of solid polymer electrolyte from $\mathrm{N}$-succinyl chitosan and lithium perchlorate. Adv. Mater. Res. 2014, 896, 58-61. [CrossRef] 
268. Fauzi, I.; Wahyuningrum, D.; Arcana, I.M. The influence of succinyl groups and lithium perchlorate on chitosan membranes as electrolyte polymers. Macromol. Symp. 2015, 353, 185-190. [CrossRef]

269. Muhammad, F.H.; Fadzil, A.F.M.; Tan, W. FTIR and electrical studies of hexanoyl chitosan-based nanocomposite polymer electrolytes. Adv. Mater. Res. 2014, 1043, 36-39. [CrossRef]

270. Muhammad, F.H.; Subban, R.H.Y.; Winie, T. Structural and electrical characterization of hexanoyl chitosan- $\mathrm{LiClO}_{4}-\mathrm{TiO}_{2}-\mathrm{DMC}$ polymer electrolytes. Key Eng. Mater. 2014, 594-595, 608-612.

271. Khiar, A.S.A.; Radzi, S.M.; Razak, N.A. Effect of $\mathrm{LiCF}_{3} \mathrm{SO}_{3}$ on L-chitosan/PMMA blend polymer electrolytes. Mol. Cryst. Liq. Cryst. 2014, 603, 66-72. [CrossRef]

272. Pandey, R.P.; Shahi, V.K. A N-o-sulphonic acid benzyl chitosan (NSBC) and N,N-dimethylene phosphonic acid propylsilane graphene oxide (NMPSGO) based multi-functional polymer electrolyte membrane with enhanced water retention and conductivity. RSC Adv. 2014, 4, 57200-57209. [CrossRef]

273. Arifin, N.A.; Khiar, A.S.A. Effect of BMITFSI to the electrical properties of methycelloluse/chitosan/ $\mathrm{NH}_{4}$ TF-based polymer electrolyte. Proc. SPIE Int. Soc. Opt. Eng. 2015, 9668, 1-7.

274. Shukur, M.F.; Kadir, M.F.Z. Hydrogen ion conducting starch-chitosan blend based electrolyte for application in electrochemical devices. Electrochim. Acta 2015, 158, 152-165. [CrossRef]

275. Chupp, J.; Shellikeri, A.; Palui, G.; Chatterjee, J. Chitosan-based gel film electrolytes containing ionic liquid and lithium salt for energy storage applications. J. Appl. Polym. Sci. 2015, 132, 1-8. [CrossRef]

276. Shamsudin, I.J.; Ahmad, A.; Hassan, N.H.; Kaddami, H. Bifunctional ionic liquid in conductive biopolymer based on chitosan for electrochemical devices application. Solid State Ion. 2015, 278, 11-19. [CrossRef]

277. Muhammad, F.H.; Azmar, A.; Winie, T. Transport properties of hexanoyl chitosan- $\mathrm{LiClO}_{4}-\mathrm{TiO}_{2}$ composite polymer electrolyte. AIP Conf. Proc. 2015, 1674, 1-6.

278. Datta, R.S.; Said, S.M.; Shahrir, S.R.; Abdullah, N.; Sabri, M.F.M.; Balamurugan, S.; Miyazaki, Y.; Hayashi, K.; Hashim, N.A.; Habiba, U.; et al. Ionic liquid entrapment by electrospun polymer nanofibre matrix as a high conductivity polymer electrolyte. RSC Adv. 2015, 5, 48217-48223. [CrossRef]

279. Aziz, S.B.; Abidin, Z.H.Z. Ion-transport study in nanocomposite solid polymer electrolytes based on chitosan: Electrical and dielectric analysis. J. Appl. Polym. Sci. 2015, 132, 1-10. [CrossRef]

280. Abu Bakar, N.Y.; Muhamaruesa, N.H.M.; Aniskari, N.A.B.; Isa, M.I.N.M. Electrical studies of carboxy methycellulose-chitosan blend biopolymer doped dodecyltrimethyl ammonium bromide solid electrolytes. Am. J. Appl. Sci. 2015, 12, 40-46. [CrossRef]

281. Buraidah, M.H.; Teo, L.P.; Au Yong, C.M.; Shah, S.; Arof, A.K. Performance of polymer electrolyte based on chitosan blended with poly(ethylene oxide) for plasmonic dye-sensitized solar cell. Opt. Mater. 2016, 57, 202-211. [CrossRef]

282. Sudaryanto; Yulianti, E.; Patimatuzzohrah. Structure and properties of solid polymer electrolyte based on chitosan and $\mathrm{ZrO}_{2}$ nanoparticle for lithium ion battery. AIP Conf. Proc. 2016, 1710, 1-10.

283. Kalaiselvimary, J.; Pradeepa, P.; Sowmya, G.; Edwinraj, S.; Ramesh Prabhu, M. Electrical characterization of proton conducting polymer electrolyte based on bio polymer with acid dopant. AIP Conf. Proc. 2016, 1728, 1-4.

284. Yusuf, S.N.F.; Azzahari, A.D.; Yahya, R.; Majid, S.R.; Careem, M.A.; Arof, A.K. From crab shell to solar cell: A gel polymer electrolyte based on $N$-phthaloylchitosan and its application in dye-sensitized solar cells. RSC Adv. 2016, 6, 27714-27724. [CrossRef]

285. Fadzallah, I.A.; Noor, I.M.; Careem, M.A.; Arof, A.K. Investigation of transport properties of chitosan-based electrolytes utilizing impedance spectroscopy. Ionics 2016, 22, 1635-1645. [CrossRef]

286. Shirdast, A.; Sharif, A.; Abdollahi, M. Effect of the incorporation of sulfonated chitosan/sulfonated graphene oxide on the proton conductivity of chitosan membranes. J. Power Sources 2016, 306, 541-551. [CrossRef]

287. Aziz, S. Role of dielectric constant on ion transport: Reformulated Arrhenius equation. Adv. Mater. Sci. Eng. 2016, 2016, 1-11. [CrossRef]

288. Alves, R.; Sentanin, F.; Sabadini, R.C.; Pawlicka, A.; Silva, M.M. Influence of cerium triflate and glycerol on electrochemical performance of chitosan electrolytes for electrochromic devices. Electrochim. Acta 2016, 217, 108-116. [CrossRef]

289. Alves, R.; Donoso, J.P.; Magon, C.J.; Silva, I.D.A.; Pawlicka, A.; Silva, M.M. Solid polymer electrolytes based on chitosan and containing europium triflate. J. Rare Earths 2016, 432, 307-312. 
290. Aziz, S.; Abdullah, O.G.; Rasheed, M.A.; Ahmed, H.M. Effect of high salt concentration (HSC) on structural, morphological, electrical characteristics of chitosan based solid polymer electrolytes. Polymers 2017, 9, 187. [CrossRef] [PubMed]

291. Pasini Cabello, S.D.; Ochoa, N.A.; Takara, E.A.; Mollá, S.; Compañ, V. Influence of pectin as a green polymer electrolyte on the transport properties of chitosan-pectin membranes. Carbohydr. Polym. 2017, 157, 1759-1768. [CrossRef] [PubMed]

292. Wang, J.; Song, S.; Gao, S.; Ravi, M.; Liu, R.; Ma, Q. Mg-ion conducting gel polymer electrolyte membranes containing biodegradable chitosan: Preparation, structural, electrical and electrochemical properties. Polym. Test. 2017, 62, 278-286. [CrossRef]

293. Muhammad, F.H.; Jamal, A.; Winie, T. Study on factors governing the conductivity performance of acylated chitosan-NaI electrolyte system. Ionics 2017, 23, 3045-3056. [CrossRef]

294. Aziz, S.; Rasheed, M.A.; Abidin, Z.H.Z. Optical and electrical characteristics of silver ion conducting nanocomposite solid polymer electrolytes based on chitosan. J. Electron. Mater. 2017, 46, 6119-6130. [CrossRef]

295. Alves, R.; Sentanin, F.; Sabadini, R.C.; Pawlicka, A.; Silva, M.M. Innovative electrolytes based on chitosan and thulium for solid state applications: Synthesis, structural, thermal characterization. J. Electroanal. Chem. 2017, 788, 156-164. [CrossRef]

296. Leones, R.; Reis, P.M.; Sabadini, R.C.; Ravaro, L.P.; Silva, I.D.A.; Camargo, A.S.S.D.; Donoso, J.P.; Magon, C.J.; Esperanca, J.M.S.S.; Pawlicka, A.; et al. A luminescent europium ionic liquid to improve the performance of chitosan polymer electrolytes. Electrochim. Acta 2017, 240, 474-485. [CrossRef]

297. Leones, R.; Sabadini, R.C.; Esperança, J.M.S.S.; Pawlicka, A.; Silva, M.M. Effect of storage time on the ionic conductivity of chitosan-solid polymer electrolytes incorporating cyano-based ionic liquids. Electrochim. Acta 2017, 232, 22-29. [CrossRef]

298. Leones, R.; Sabadini, R.C.; Esperança, J.M.S.S.; Pawlicka, A.; Silva, M.M. Playing with ionic liquids to uncover novel polymer electrolytes. Solid State Ion. 2017, 300, 46-52. [CrossRef]

299. Yang, Y.; Hu, H.; Zhou, C.H.; Xu, S.; Sebo, B.; Zhao, X.Z. Novel agarose polymer electrolyte for quasi-solid state dye-sensitized solar cell. J. Power Sources 2011, 196, 2410-2415. [CrossRef]

300. Wang, W.; Guo, X.; Yang, Y. Lithium iodide effect on the electrochemical behavior of agarose based polymer electrolyte for dye-sensitized solar cell. Electrochim. Acta 2011, 56, 7347-7351. [CrossRef]

301. Lima, E.; Raphael, E.; Sentanin, F.; Rodrigues, L.C.; Ferreira, R.A.S.; Carlos, L.D.; Silva, M.M.; Pawlicka, A. Photoluminescent polymer electrolyte based on agar and containing europium picrate for electrochemical devices. Mater. Sci. Eng. B 2012, 177, 488-493. [CrossRef]

302. Leones, R.; Sentanin, F.; Rodrigues, L.C.; Marrucho, I.M.; Esperanca, J.M.S.S.; Pawlicka, A.; Silva, M.M. Investigation of polymer electrolytes based on agar and ionic liquids. Express Polym. Lett. 2012, 6, 1007-1016. [CrossRef]

303. Yang, Y.; Yi, P.; Zhou, C.; Cui, J.; Zheng, X.; Xiao, S.; Guo, X.; Wang, W. Magnetic field processed solid-state dye-sensitized solar cells with nickel oxide modified agarose electrolyte. J. Power Sources 2013, 243, 919-924. [CrossRef]

304. Guo, X.Y.; Yi, P.F.; Wang, W.J.; Xiao, S.; Yang, Y. Effects of polyethylene glycol on agarose-based magnetic polymer electrolyte for dye-sensitized solar cell. Adv. Mater. Res. 2013, 652-654, 860-864. [CrossRef]

305. Guo, X.; Yi, P.; Yang, Y.; Cui, J.; Xiao, S.; Wang, W. Effects of surfactants on agarose-based magnetic polymer electrolyte for dye-sensitized solar cells. Electrochim. Acta 2013, 90, 524-529. [CrossRef]

306. Koh, J.C.H.; Ahmad, Z.A.; Mohamad, A.A. Bacto agar-based gel polymer electrolyte. Ionics 2012, 18, 359-364. [CrossRef]

307. Alves, R.D.; Rodrigues, L.C.; Andrade, J.R.; Pawlicka, A.; Pereira, L.; Martins, R.; Fortunato, E.; Silva, M.M. Study and characterization of a novel polymer electrolyte based on agar doped with magnesium triflate. Mol. Cryst. Liq. Cryst. 2013, 570,1-11. [CrossRef]

308. Yang, Y.; Cui, J.; Yi, P.; Zheng, X.; Guo, X.; Wang, W. Effects of nanoparticle additives on the properties of agarose polymer electrolytes. J. Power Sources 2014, 248, 988-993. [CrossRef]

309. Boopathi, G.; Pugalendhi, S.; Selvasekarapandian, S.; Premalatha, M.; Monisha, S.; Aristatil, G. Development of proton conducting biopolymer membrane based on agar-agar for fuel cell. Ionics 2016, 23, 1-10. [CrossRef] 
310. Raphael, E.; Jara, D.H.; Schiavon, M.A. Optimizing photovoltaic performance in CuInS 2 and CdS quantum dot-sensitized solar cells by using an agar-based gel polymer electrolyte. RSC Adv. 2017, 7, 6492-6500. [CrossRef]

311. Selvalakshmi, S.; Vijaya, N.; Selvasekarapandian, S.; Premalatha, M. Biopolymer agar-agar doped with $\mathrm{NH}_{4} \mathrm{SCN}$ as solid polymer electrolyte for electrochemical cell application. J. Appl. Polym. Sci. 2017, 134, 1-10. [CrossRef]

312. Selvalakshmi, S.; Mathavan, T.; Selvasekarapandian, S.; Premalatha, M. Study on $\mathrm{NH}_{4} \mathrm{I}$ composition effect in agar-agar-based biopolymer electrolyte. Ionics 2017, 23, 1-7. [CrossRef]

313. Nadia, S.R.; Khanmirzaei, M.H.; Ramesh, S.; Ramesh, K. Quasi-solid-state agar-based polymer electrolytes for dye-sensitized solar cell applications using imidazolium-based ionic liquid. Ionics 2017, 23, 1585-1590. [CrossRef]

314. Shamsudin, I.J.; Ahmad, A.; Hassan, N.H.; Kaddami, H. Solid biopolymer electrolyte based on K-Carrageenan for electrochemical devices application. Asian J. Chem. 2014, 26, 77-80. [CrossRef]

315. Jumaah, F.N.; Mobarak, N.N.; Ahmad, A.; Ghani, M.A.; Rahman, M.Y.A. Derivative of iota-carrageenan as solid polymer electrolyte. Ionics 2015, 21, 1311-1320. [CrossRef]

316. Rudhziah, S.; Ahmad, A.; Ahmad, I.; Mohamed, N.S. Biopolymer electrolytes based on blend of kappa-carrageenan and cellulose derivatives for potential application in dye sensitized solar cell. Electrochim. Acta 2015, 175, 1-7. [CrossRef]

317. Chan, S.; Bantang, J.P.; Camacho, D. Influence of nanomaterial fillers in biopolymer electrolyte system for squaraine-based dye-sensitized solar cells. Int. J. Electrochem. Sci. 2015, 10, 7696-7706.

318. Rudhziah, S.; Ahmad, A.; Mohamed, N.S. The effect of lithium iodide to the properties of carboxymethyl $\mathrm{K}$-carrageenan/carboxymethyl cellulose polymer electrolyte and dye-sensitized solar cell performance. Polymers 2016, 8, 1-10.

319. Karthikeyan, S.; Selvasekarapandian, S.; Premalatha, M.; Monisha, S.; Boopathi, G.; Aristatil, G.; Arun, A.; Madeswaran, S. Proton-conducting I-Carrageenan-based biopolymer electrolyte for fuel cell application. Ionics 2016, 23, 1-6. [CrossRef]

320. Pérez-Madrigal, M.M.; Estrany, F.; Armelin, E.; Díaz, D.D.; Alemán, C. Towards sustainable solid-state supercapacitors: Electroactive conducting polymers combined with biohydrogels. J. Mater. Chem. A 2016, 4, 1792-1805. [CrossRef]

321. Moniha, V.; Alagar, M.; Selvasekarapandian, S.; Sundaresan, B.; Boopathi, G. Conductive bio-polymer electrolyte iota-carrageenan with ammonium nitrate for application in electrochemical devices. J. Non-Cryst. Solids 2018, 481, 424-434. [CrossRef]

322. Mishra, R.K.; Datt, M.; Banthia, A.K.; Majeed, A.B.A. Development of novel pectin based membranes as proton conducting material. Int. J. Plast. Technol. 2012, 16, 80-88. [CrossRef]

323. Leones, R.; Botelho, M.B.S.; Sentanin, F.; Cesarino, I.; Pawlicka, A.; Camargo, A.S.S.; Silva, M.M. Pectin-based polymer electrolytes with Ir(III) complexes. Mol. Cryst. Liq. Cryst. 2014, 604, 117-125. [CrossRef]

324. Vijaya, N.; Selvasekarapandian, S.; Sornalatha, M.; Sujithra, K.S.; Monisha, S. Proton-conducting biopolymer electrolytes based on pectin doped with $\mathrm{NH}_{4} \mathrm{X}(\mathrm{X}=\mathrm{Cl}, \mathrm{Br})$. Ionics 2016, 23, 1-10. [CrossRef]

325. Mendes, J.P.; Esperança, J.M.S.S.; Medeiros, M.J.; Pawlicka, A.; Silva, M.M. Structural, morphological, ionic conductivity, thermal properties of pectin-based polymer electrolytes. Mol. Cryst. Liq. Cryst. 2017, 643, 266-273. [CrossRef]

326. Khalid, M.; Hartono, A.M.B. Ionically conducting and environmentally safe gum Arabic as a high-performance gel-like electrolyte for solid-state supercapacitors. J. Solid State Electrochem. 2017, 21, 2443-2447. [CrossRef]

327. Mattos, R.I.; Pawlicka, A.; Tambelli, C.E.; Donoso, J.P. NMR and conductivity study of gelatin-based polymer electrolytes. Mol. Cryst. Liq. Cryst. 2008, 483, 120-129. [CrossRef]

328. Silva, M.M.; Barbosa, P.C.; Rodrigues, L.C. New developments in conducting polymers based on commercial gelatin. ECS Trans. 2009, 16, 413-419.

329. Silva, M.M.; Barbosa, P.C.; Rodrigues, L.C.; Goncalves, A.; Costa, C.; Fortunato, E. Gelatin in electrochromic devices. Opt. Mater. 2010, 32, 719-722. [CrossRef]

330. Li, Q.; Wu, J.; Tang, Z.; Xiao, Y.; Huang, M.; Lin, J. Application of poly(acrylic acid-g-gelatin)/polypyrrole gel electrolyte in flexible quasi-solid-state dye-sensitized solar cell. Electrochim. Acta 2010, 55, 2777-2781. [CrossRef] 
331. Mattos, R.I.; Pawlicka, A.; Lima, J.F.; Tambelli, C.E.; Magon, C.J.; Donoso, J.P. Magnetic resonance and conductivity study of gelatin-based proton conductor polymer electrolytes. Electrochim. Acta 2010, 55, 1396-1400. [CrossRef]

332. Vieira, D.F.; Pawlicka, A. Optimization of performances of gelatin/LiBF 4 -based polymer electrolytes by plasticizing effects. Electrochim. Acta 2010, 55, 1489-1494. [CrossRef]

333. Lima, E.; Mattos, R.; Sentanin, F.; Rodrigues, L.C.; Silva, M.M.; Ferreira, R.A.S.; Carlos, L.D.; Pawlicka, A. Functional novel polymer electrolytes containing europium picrate. Mater. Res. Innov. 2011, 15, 1-6. [CrossRef]

334. Pawlicka, A.; Mattos, R.I.; Lima, J.F.; Tambelli, C.E.; Magon, C.J.; Donoso, J.P. Magnetic resonance and conductivity study of a gelatin-based polymer gel electrolyte. Electrochim. Acta 2011, 57, 187-191. [CrossRef]

335. Leones, R.; Sentanin, F.; Rodrigues, L.C.; Ferreira, R.A.S.; Marrucho, I.M.; Esperanca, J.M.S.S.; Pawlicka, A.; Carlos, L.D.; Silva, M.M. Novel polymer electrolytes based on gelatin and ionic liquids. Opt. Mater. 2012, 35, 187-195. [CrossRef]

336. Ponez, L.; Sentanin, F.C.; Majid, S.R.; Arof, A.K.; Pawlicka, A. Ion-conducting membranes based on gelatin and containing LiI/I $\mathrm{I}_{2}$ for electrochromic devices. Mol. Cryst. Liq. Cryst. 2012, 554, 239-251. [CrossRef]

337. Basu, T.; Goswami, M.M.; Middya, T.R.; Tarafdar, S. Morphology and ion-conductivity of gelatin- $\mathrm{LiClO}_{4}$ films: Fractional diffusion analysis. J. Phys. Chem. B 2012, 116, 11362-11369. [CrossRef]

338. Basu, T.; Giri, A.; Tarafdar, S.; Das, S. Electrical impedance response of gamma irradiated gelatin based solid polymer electrolytes analyzed using a generalized calculus formalism. J. Electroanal. Chem. 2015, 755, 52-62. [CrossRef]

339. Basu, T.; Middya, T.R.; Tarafdar, S. Ion-conductivity study and anomalous diffusion analysis of plasticized gelatin films. J. Appl. Polym. Sci. 2013, 130, 3018-3024. [CrossRef]

340. Alves, R.D.; Rodrigues, L.C.; Andrade, J.R.; Fernandes, M.; Pinto, J.V.; Pereira, L.; Pawlicka, A.; Martins, R.; Fortunato, E.; Bermudez, V.D.Z.; et al. Gelatin $\mathrm{Zn}\left(\mathrm{CF}_{3} \mathrm{SO}_{3}\right)_{2}$ polymer electrolytes for electrochromic devices. Electroanalysis 2013, 25, 1483-1490. [CrossRef]

341. Ramadan, R.; Kamal, H.; Hashem, H.M.; Abdel-Hady, K. Gelatin-based solid electrolyte releasing Li+ for smart window applications. Sol. Energy Mater. Sol. Cells 2014, 127, 147-156. [CrossRef]

342. Sharma, S.; Khannam, M.; Boruah, M.; Nath, B.C.; Dolui, S.K. Development of dye-sensitized solar cells based on gold/gelatin gel electrolyte: Effect of different aspect ratio of gold nanocrystals. IEEE J. Photovolt. 2015, 5, 1665-1673. [CrossRef]

343. Leones, R.; Sabadini, R.C.; Sentanin, F.C.; Esperança, J.M.S.S.; Pawlicka, A.; Silva, M.M. Polymer electrolytes for electrochromic devices through solvent casting and sol-gel routes. Sol. Energy Mater. Sol. Cells 2017, 169, 98-106. [CrossRef]

344. Idris, R.; Glasse, M.D.; Latham, R.J.; Linford, R.G.; Schlindwein, W.S. Polymer electrolytes based on modified natural rubber for used in rechargeable lithium batteries. J. Power Sources 2001, 94, 206-211. [CrossRef]

345. Glasse, M.D.; Idris, R.; Latham, R.J.; Linford, R.G.; Schlindwein, W.S. Polymer electrolytes based on modified natural rubber. Solid State Ion. 2002, 147, 289-294. [CrossRef]

346. Kumutha, K.; Alias, Y.; Said, R. FTIR and thermal studies of modified natural rubber based polymer electrolytes. Ionics 2005, 11, 472-476. [CrossRef]

347. Aziz, M.; Latif, F.; Chew, C.L.; Katun, N. The impedance spectroscopy studies of PVC/ENR 50/LiCF $3 \mathrm{SO}_{3}$. Solid State Phenom. 2006, 111, 67-70. [CrossRef]

348. Ali, A.M.M.; Yahya, M.Z.A.; Bahron, H.; Subban, R.H.Y. Electrochemical studies on polymer electrolytes based on poly(methyl methacrylate)-grafted natural rubber for lithium polymer battery. Ionics 2006, 12, 303-307. [CrossRef]

349. Latif, F.; Aziz, M.; Ali, A.M.M.; Yahya, M.Z.A. The coagulation impact of $50 \%$ epoxidised natural rubber chain in ethylene carbonate-plasticized solid electrolytes. Macromol. Symp. 2009, 277, 62-68. [CrossRef]

350. Kamisan, A.S.; Kudin, T.I.T.; Ali, A.M.M.; Yahya, M.Z.A. Gel polymer electrolyte based on methyl-grafted natural rubber for proton batteries. Mater. Res. Innov. 2009, 13, 263-265. [CrossRef]

351. Su'ait, M.S.; Ahmad, A.; Hamzah, H.; Rahman, M.Y.A. Preparation and characterization of PMMA-MG49- $\mathrm{LiClO}_{4}$ solid polymeric electrolyte. J. Phys. D. Appl. Phys. 2009, 42, 1-5.

352. Low, S.P.; Ahmad, A.; Rahman, M.Y.A. Effect of ethylene carbonate plasticizer and $\mathrm{TiO}_{2}$ nanoparticles on $49 \%$ poly(methyl methacrylate) grafted natural rubber-based polymer electrolyte. Ionics 2010, 16, 821-826. [CrossRef] 
353. Kamisan, A.S.; Kudin, T.I.T.; Ali, A.M.M.; Yahya, M.Z.A. Electrical and physical studies on $49 \%$ methyl-grafted natural rubber-based composite polymer gel electrolytes. Electrochim. Acta 2011, 57, 207-211. [CrossRef]

354. Ahmad, A.; Rahman, M.Y.A.; Su'ait, M.S.; Hamzah, H. Study of MG49-PMMA based solid polymer electrolyte. Open Mater. Sci. J. 2011, 5, 170-177. [CrossRef]

355. Kamisan, A.S.; Kudin, T.I.T.; Ali, A.M.M.; Yahya, M.Z.A. Polymer gel electrolytes based on $49 \%$ methyl-grafted natural rubber. Sains Malays. 2011, 40, 49-54.

356. Zaki, N.H.M.; Mahmud, Z.S.; Adam, N.I.; Ahmad, A.H.; Ali, A.M.M.; Yahya, M.Z.A. Characterization of plasticized grafted natural rubber-30\% poly(methyl methacrylate) (MG30) based polymer electrolytes. In Proceedings of the 2012 IEEE Symposium on Business, Engineering and Industrial Applications, Bandung, Indonesia, 23-26 September 2012; pp. 705-708.

357. Rahman, M.Y.A.; Ahmad, A.; Lee, T.K.; Farina, Y.; Dahlan, H.M. $\mathrm{LiClO}_{4}$ salt concentration effect on the properties of PVC-modified low molecular weight LENR50-based solid polymer electrolyte. Polym. Polym. Compos. 2012, 124, 2227-2233. [CrossRef]

358. Zainal, N.; Mohamed, N.S.; Idris, R. Properties of ENR-50 based electrolyte system. Sains Malays. 2013, 42, 481-485.

359. Jafirin, S.; Ahmad, I.; Ahmad, A. Potential use of cellulose from kenaf in polymer electrolytes based on MG49 rubber composites. BioResources 2013, 8, 5947-5964. [CrossRef]

360. Ali, A.M.M.; Subban, R.H.Y.; Bahron, H.; Yahya, M.Z.A.; Kamisan, A.S. Investigation on modified natural rubber gel polymer electrolytes for lithium polymer battery. J. Power Sources 2013, 244, 636-640. [CrossRef]

361. Ataollahi, N.; Ahmad, A.; Hamzah, H.; Rahman, M.Y.; Mohamed, N.S. Effects of $\mathrm{LiCF}_{3} \mathrm{SO}_{3}$ on PVDF-HFP/MG49 (70/30) based blend polymer electrolyte. Appl. Mech. Mater. 2013, 313-314, 117-120. [CrossRef]

362. Ataollahi, N.; Ahmad, A.; Hamzah, H.; Rahman, M.Y.A.; Mohamed, N.S. Ionic conduction of blend poly(vinylidene fluoride-hexafluoro propylene) and poly(methyl methacrylate)-grafted natural rubber based solid polymer electrolyte. Int. J. Electrochem. Sci. 2013, 8, 7875-7884.

363. TianKhoon, L.; Ataollahi, N.; Hassan, N.H.; Ahmad, A. Studies of porous solid polymeric electrolytes based on poly(vinylidene fluoride) and poly(methyl methacrylate) grafted natural rubber for applications in electrochemical devices. J. Solid State Electrochem. 2016, 20, 203-213. [CrossRef]

364. Idris, R.; Bujang, N.H. Epoxidised natural rubber based polymer electrolyte systems for electrochemical device applications. Adv. Mater. Res. 2014, 896, 62-65. [CrossRef]

365. Ataollahi, N.; Ahmad, A.; Lee, T.K.; Abdullah, A.R.; Rahman, M.Y.A. Preparation and characterization of PVDF-MG49- $\mathrm{NH}_{4} \mathrm{CF}_{3} \mathrm{SO}_{3}$ based solid polymer electrolyte. e-Polymers 2014, 14, 115-120. [CrossRef]

366. Jafirin, S.; Ahmad, I.; Ahmad, A. Carboxymethyl cellulose from kenaf reinforced composite polymer electrolytes based $49 \%$ poly(methyl methacrylate) -grafted natural rubber. Malays. J. Anal. Sci. 2014, 18, 376-384.

367. Tiankhoon, L.; Hassan, N.H.; Rahman, M.Y.A.; Vedarajan, R.; Matsumi, N.; Ahmad, A. One-pot synthesis nano-hybrid $\mathrm{ZrO}_{2}-\mathrm{TiO}_{2}$ fillers in $49 \%$ poly(methyl methacrylate) grafted natural rubber (MG49) based nano-composite polymer electrolyte for lithium ion battery application. Solid State Ion. 2015, 276, 72-79. [CrossRef]

368. Nazir, K.; Aziz, A.F.; Adam, N.I.; Yahya, M.Z.A.; Ali, A.M.M. Effect of epoxidation on 30\% poly(methyl methacrylate)-grafted natural rubber polymer electrolytes. AIP Conf. Proc. 2015, 1674, 1-6.

369. Chan, C.H.; Kammer, H.W. Impedance spectroscopy of polymer electrolytes based on epoxidized natural rubber with $50 \mathrm{~mol} \%$ epoxide content. Polym. Eng. Sci. 2015, 55, 2250-2255. [CrossRef]

370. Jamal, A.; Muhammad, F.H.; Ali, A.M.M.; Winie, T. Blends of hexanoyl chitosan/epoxidized natural rubber doped with EMImTFSI. Ionics 2017, 23, 357-366. [CrossRef]

371. Chan, C.H.; Kammer, H.-W. Impedance spectra of polymer electrolytes. Ionics 2017, 23, 2327-2337. [CrossRef]

372. Zimmermann, J.; Jürgensen, N.; Morfa, A.J.; Wang, B.; Tekoglu, S.; Hernandez-Sosa, G. Poly(lactic-co-glycolic acid) (PLGA) as ion-conducting polymer for biodegradable light-emitting electrochemical cells. ACS Sustain. Chem. Eng. 2016, 4, 7050-7055. [CrossRef]

373. Neto, M.J.; Sentanin, F.; Esperanca, J.M.S.S.; Medeiros, M.J.; Pawlicka, A.; Bermudez, V.D.Z.; Silva, M.M. Gellan gum-Ionic liquid membranes for electrochromic device application. Solid State Ion. 2015, 274, 64-70. [CrossRef] 
374. Singh, R.; Bhattacharya, B.; Rhee, H.W.; Singh, P.K. Solid gellan gum polymer electrolyte for energy application. Int. J. Hydrogen Energy 2015, 40, 9365-9372. [CrossRef]

375. Sudhakar, Y.N.; Selvakumar, M.; Krishna Bhat, D. Effect of acid dopants in biodegradable gel polymer electrolyte and the performance in an electrochemical double layer capacitor. Phys. Scr. 2015, 90, 1-12. [CrossRef]

376. Singh, R.; Polu, A.R.; Bhattacharya, B.; Rhee, H.W.; Varlikli, C.; Singh, P.K. Perspectives for solid biopolymer electrolytes in dye sensitized solar cell and battery application. Renew. Sustain. Energy Rev. 2016, 65, 1098-1117. [CrossRef]

377. O’Regan, B.; Gratzel, M. A low-cost, high-efficiency solar cell based on dye sensitized colloidal TiO 2 films. Nature 1991, 353, 737-740. [CrossRef]

378. Wu, J.; Lan, Z.; Hao, S.; Li, P.; Lin, J.; Huang, M.; Fang, L.; Huang, Y. Progress on the electrolytes for dye-sensitized solar cells. Pure Appl. Chem 2008, 80, 2241-2258. [CrossRef]

379. Adedokun, O.; Titilope, K.; Awodugba, A.O. Review on natural dye-sensitized solar cells (DSSCs). Int. J. Eng. Technol. 2016, 2, 34-41. [CrossRef]

380. Alias, S.S.; Mohamad, A.A. Effect of $\mathrm{NH}_{4} \mathrm{I}$ and $\mathrm{I}_{2}$ concentration on agar gel polymer electrolyte properties for a dye-sensitized solar cell. Ionics 2013, 19, 1185-1194. [CrossRef]

381. Hsu, H.L.; Tien, C.F.; Yang, Y.T.; Leu, J. Dye-sensitized solar cells based on agarose gel electrolytes using allylimidazolium iodides and environmentally benign solvents. Electrochim. Acta 2013, 91, 208-213. [CrossRef]

382. Singh, R.; Jadhav, N.A.; Majumder, S.; Bhattacharya, B.; Singh, P.K. Novel biopolymer gel electrolyte for dye-sensitized solar cell application. Carbohydr. Polym. 2013, 91, 682-685. [CrossRef] [PubMed]

383. Ibrahim, S.; Ahmad, A.; Mohamed, N.S. Comprehensive studies on polymer electrolyte and dye-sensitized solar cell developed using castor oil-based polyurethane. J. Solid State Electrochem. 2018, 22, 461-470. [CrossRef]

384. Su'ait, M.S.; Jumaah, F.N.; Faizzi, H.M.; Mamat, S.; Ludin, N.A.; Farhan, W.A.; Haron, A.; Atifah, N.; Latif, M.N.; Badri, K.H.; et al. Palm-based polyurethane-ionic liquid gel polymer electrolyte for quasi-solid state dye sensitized solar cell. Ind. Crop. Prod. 2018, 113, 406-413. [CrossRef] 